\title{
Two-loop beta function for complex scalar electroweak multiplets
}

\author{
Joachim Brod and Zachary Polonsky \\ Department of Physics, University of Cincinnati, \\ Cincinnati, OH 45221, U.S.A. \\ E-mail: joachim.brod@uc.edu, polonsza@mail.uc.edu
}

\begin{abstract}
We present the general form of the renormalizable four-point interactions of a complex scalar field furnishing an irreducible representation of $\mathrm{SU}(2)$, and derive a set of algebraic identities that facilitates the calculation of higher-order radiative corrections. As an application, we calculate the two-loop beta function for the SM extended by a scalar multiplet, and provide the result explicitly in terms of the group invariants. Our results include the evolution of the Higgs-portal couplings, as well as scalar "minimal dark matter". We present numerical results for the two-loop evolution of the various couplings.
\end{abstract}

KEYwords: Renormalization Group, Gauge Symmetry

ARXIV EPRINT: 2007.13755 


\section{Contents}

1 Introduction 1

2 Construction of the operator basis $\quad 2$

3 Beta function for a scalar multiplet 5

4 Group theory relations $\quad 16$

5 Numerics 26

$\begin{array}{llr}6 & \text { Conclusions } & 29\end{array}$

$\begin{array}{ll}\text { A Analytic checks of our calculation } & 30\end{array}$

B Renormalization constants $\quad 31$

\section{Introduction}

Complex scalar fields furnishing a general representation of the electroweak gauge group $\mathrm{SU}(2) \times \mathrm{U}(1)$ of the standard model $(\mathrm{SM})$ received increased interest in recent years. For instance, they can provide a viable dark matter candidate in so-called minimal dark-matter models [1].

The renormalization group (RG) evolution of coupling constants is an invaluable tool in phenomenological analyses [2]. It plays a particularly important role when interpreting and comparing the results of experiments performed at widely different energy scales, such as dark matter direct detection and production of dark matter at particle colliders. A framework for consistent RG analysis for fermionic dark matter in the context of effective field theories has been presented in ref. [3]. The first consistent and complete basis of effective operators for scalar dark matter up to mass dimension six has been written down in ref. [4]; however, the RG evolution has not yet been calculated.

For scalar dark matter it is possible to write down self interactions, as well as interactions with the SM, at the renormalizable level - the so-called Higgs-portal dark matter [5-7]. To our knowledge, the first classification of the self interactions of scalar fields with electroweak charges has been given in ref. [8]. In this work, we rederive the scalar potential in a slightly different form that is well suited for the calculation of radiative corrections.

We then calculate the beta functions for all scalar couplings, as well as the new scalar contributions to all SM couplings, at the two-loop level. To this end, we prove a set of algebraic relations that allows to express all two-loop matrix elements in terms of treelevel matrix elements of the basis operators in the scalar potential. While these algebraic 
relations simply rely on the algebra of Clebsch-Gordan coefficients as well as $\mathrm{SU}(2)$ gauge symmetry, many of them turn out to be quite non-trivial, and have not been derived before, to the best of our knowledge. Among other results, we show how to express a product of two $\mathrm{SU}(2)$ generators, contracted over their adjoint indices, in terms of Clebsch-Gordan coefficients. The resulting relations can be used to manipulate general representations of the $\mathrm{SU}(2)$ algebra in an algorithmic way.

Our results are valid for a scalar field furnishing an arbitrary irreducible representation of $\mathrm{SU}(2)$ and for arbitrary hypercharge. While these results are known in principle [9, 10], we present them in closed form and explicitly in terms of group invariants for the first time. We believe that this form of the beta functions makes them more suitable for practical applications. Auxiliary files with our analytic results in computer-readable form are available via a gitlab repository (see section 6).

As a cross check of our calculational setup, we also computed the two-loop beta function for the SM Higgs, as well as the top, bottom, charm, and tau Yukawa couplings. We find a result consistent with the SM beta function extracted from ref. [9], see ref. [11], if we take into account the corrections pointed out in refs. [12, 13]. See also refs. [14-18] for recent results at the three- and four-loop level.

Depending on the representation, the impact of the one- and two-loop contributions to the running of the scalar as well as the SM couplings can be sizeable. We discuss a few examples, focusing on a scalar septuplet ("minimal dark matter") and the running of the SM quartic Higgs and SU(2) gauge coupling.

This paper is organized as follows. In section 2 we define our setup and construct the scalar potential. In section 3 we present our results for the beta functions. The required algebraic relations are collected and proven in section 4 . Section 5 contains numerical illustrations of our results. We conclude in section 6. Supplementary material is presented in two appendices. In appendix A we describe the various analytic checks that we performed on our calculation, and derive explicit formal expressions for the beta functions. In appendix B we provide all field and mass renormalization constants that are necessary in intermediate steps of the calculation. For completeness, we also include all quadratic poles of the coupling renormalization constants.

\section{Construction of the operator basis}

We consider a complex scalar field $\varphi$ with mass $M_{\varphi}$ which furnishes a $\left(2 j_{\varphi}+1\right)$-dimensional irreducible representation of the Standard Model $\mathrm{SU}(2) \times \mathrm{U}(1)$ gauge group, where $j_{\varphi}=0,1 / 2,1, \ldots$ is any integer or half integer. The Lagrangian for this model is given by

$$
\mathcal{L}_{\varphi}=\left(D_{\mu} \varphi\right)^{\dagger} D^{\mu} \varphi-M_{\varphi}^{2} \varphi^{\dagger} \varphi-\frac{1}{4} W_{\mu \nu}^{a} W^{a \mu \nu}-\frac{1}{4} B_{\mu \nu} B^{\mu \nu}-V_{\varphi}
$$

The summation convention over Lorentz and adjoint gauge indices is in use here and in the following. The covariant derivative acting on the scalar field is given by

$$
D_{\mu} \varphi_{k}=\sum_{l}\left(\delta_{k l} \partial_{\mu}-i g_{2} \tilde{\tau}_{k l}^{a} W_{\mu}^{a}+i \frac{Y_{\varphi}}{2} g_{1} \delta_{k l} B_{\mu}\right) \varphi_{l}
$$


with the corresponding field strength tensors

$$
W_{\mu \nu}^{a}=\partial_{\mu} W_{\nu}^{a}-\partial_{\nu} W_{\mu}^{a}+g_{2} \epsilon^{a b c} W_{\mu}^{b} W_{\nu}^{c}, \quad B_{\mu \nu}=\partial_{\mu} B_{\nu}-\partial_{\nu} B_{\mu}
$$

Here, $B_{\mu}$ and $W_{\mu}^{a}$ (with $\left.a=1,2,3\right)$ are the $\mathrm{U}(1)$ and $\mathrm{SU}(2)$ gauge fields, respectively. The $\tilde{\tau}_{k l}^{a}$ are $\mathrm{SU}(2)$ generators in the $\left(2 j_{\varphi}+1\right)$-dimensional representation, defined by

$$
\left(\tilde{\tau}^{1} \pm i \tilde{\tau}^{2}\right)_{k l}=\delta_{k, l \pm 1} \sqrt{\left(j_{\varphi} \mp l\right)\left(j_{\varphi} \pm l+1\right)}, \quad\left(\tilde{\tau}^{3}\right)_{k l}=l \delta_{k, l}
$$

with $k, l$ running over the values $-j_{\varphi},-j_{\varphi}+1, \ldots, j_{\varphi}-1, j_{\varphi}$, while $Y_{\varphi}$ is the scalar hypercharge.

We now derive the general form of the scalar potential $V_{\varphi}$. Any Hermitian, renormalizable four-scalar operator has the general form

$$
\mathcal{O}_{\varphi}=\sum_{i r k s} \varphi_{i}^{*} \varphi_{k}^{*} \varphi_{r} \varphi_{s} v_{i r k s}^{j_{\varphi}}
$$

The form of the real coefficients $v_{i r k s}^{j_{\varphi}}$ must be determined such that the operator $\mathcal{O}_{\varphi}$ is invariant under the $\mathrm{SU}(2)$ gauge group (the $\mathrm{U}(1)$ invariance is immediately apparent). Ignoring all quantum numbers that do not transform under $\mathrm{SU}(2)$, the operator coefficients can be written as

$$
v_{i r k s}^{j_{\varphi}} \equiv\left\langle j_{\varphi}, r ; j_{\varphi}, s|V| j_{\varphi}, i ; j_{\varphi}, k\right\rangle
$$

where $V$ are the reduced matrix elements. Inserting two complete sets of states, we have

$$
\begin{aligned}
v_{i r k s}^{j_{\varphi}} & =\sum_{J J^{\prime}} \sum_{M M^{\prime}} C_{j_{\varphi} j_{\varphi}}(J M ; r s) C_{j_{\varphi} j_{\varphi}}\left(J^{\prime} M^{\prime} ; i k\right)\left\langle J M|V| J^{\prime} M^{\prime}\right\rangle \\
& \equiv \sum_{J J^{\prime}} \sum_{M M^{\prime}} C_{j_{\varphi} j_{\varphi}}(J M ; r s) C_{j_{\varphi} j_{\varphi}}\left(J^{\prime} M^{\prime} ; i k\right) v_{M M^{\prime}}^{J J^{\prime}},
\end{aligned}
$$

where $C_{j j^{\prime}}\left(J M ; m m^{\prime}\right)$ are Clebsch-Gordan coefficients (we use the notation of ref. [19]). Defining the composite field operator

$$
\Phi_{M}^{(J)} \equiv \sum_{m n} \varphi_{m} \varphi_{n} C_{j_{\varphi} j_{\varphi}}(J M ; m n),
$$

eq. (2.5) becomes

$$
\mathcal{O}_{\varphi}=\sum_{J J^{\prime}} \sum_{M M^{\prime}}\left(\Phi_{M}^{(J)}\right)^{*} \Phi_{M^{\prime}}^{\left(J^{\prime}\right)} v_{M M^{\prime}}^{J J^{\prime}}
$$

Writing a general $\mathrm{SU}(2)$ transformation as $D^{(J)}=\exp \left(i \theta^{a} \tilde{\tau}^{(J), a}\right)$, where $\tilde{\tau}^{(J), a}$ are here the $\mathrm{SU}(2)$ generators in the $2 J+1$-dimensional representation, gauge invariance requires

$$
\mathcal{O}_{\varphi} \rightarrow \sum_{J J^{\prime}} \sum_{M M^{\prime} N N^{\prime}}\left(\Phi_{N}^{(J)}\right)^{*} \Phi_{N^{\prime}}^{\left(J^{\prime}\right)}\left(D_{M N}^{(J)}\right)^{*} D_{M^{\prime} N^{\prime}}^{\left(J^{\prime}\right)} v_{M M^{\prime}}^{J J^{\prime}}=\mathcal{O}_{\varphi}
$$

Using the unitarity of the $D$ matrices, this can be written as the condition

$$
\sum_{M^{\prime}} v_{M M^{\prime}}^{J J^{\prime}} D_{M^{\prime} N^{\prime}}^{\left(J^{\prime}\right)}=\sum_{N} D_{M N}^{(J)} v_{N N^{\prime}}^{J J^{\prime}}
$$


By Schur's Lemma, $v$ is either zero or has the form

$$
v_{M M^{\prime}}^{J J^{\prime}}=\frac{\lambda_{\varphi}^{(J)}}{4} \delta^{J J^{\prime}} \delta_{M M^{\prime}},
$$

where $\lambda_{\varphi}^{(J)}$ is a constant. We define a set of "Sigma matrices" as

$$
\Sigma_{m n}^{(J), M} \equiv C_{j_{\varphi} j_{\varphi}}(J M, m n),
$$

(note that we regard the isospin $j_{\varphi}$ of the scalar multiplet to be fixed in this work). We then write the general potential as ${ }^{1}$

$$
V_{\varphi}[\varphi]=\sum_{J} \frac{\lambda_{\varphi}^{(J)}}{4} \sum_{M}\left|\sum_{m n} \varphi_{m} \Sigma_{m n}^{(J), M} \varphi_{n}\right|^{2} .
$$

The symmetry properties of the Clebsch-Gordan coefficients imply the corresponding properties of the Sigma matrices,

$$
\Sigma_{m m^{\prime}}^{(J), a}=(-1)^{J-2 j_{\varphi}} \Sigma_{m^{\prime} m}^{(J), a}
$$

This restricts the number of independent operators in the basis. Obviously, the coefficients $v_{i r k s}^{j_{\varphi}}$ in eq. (2.5) can be chosen symmetric under exchange of $i \leftrightarrow k$ and $r \leftrightarrow s$. Hence, the only non-zero operators in our basis are those involving Sigma matrices that are symmetric in their lower indices,

$$
\Sigma_{m m^{\prime}}^{(J) a}=\Sigma_{m^{\prime} m}^{(J) a}
$$

This immediately tells us that there are $N_{\varphi} \equiv$ floor $\left(j_{\varphi}+1\right)$ operators in our basis. As a related consequence, the sum over $J$ in eq. (2.14) effectively runs only over even values for integer $j_{\varphi}$, while for half-integer $j_{\varphi}$ only terms with odd $J$ contribute.

We illustrate this construction by the example of an electroweak doublet. The Sigma matrices for $j_{\varphi}=1 / 2$ are

$$
\Sigma^{(0), 0}=\frac{1}{\sqrt{2}}\left(\begin{array}{cc}
0 & 1 \\
-1 & 0
\end{array}\right) ; \quad \Sigma^{(1), 1}=\left(\begin{array}{ll}
1 & 0 \\
0 & 0
\end{array}\right), \quad \Sigma^{(1), 0}=\frac{1}{\sqrt{2}}\left(\begin{array}{ll}
0 & 1 \\
1 & 0
\end{array}\right), \quad \Sigma^{(1),-1}=\left(\begin{array}{ll}
0 & 0 \\
0 & 1
\end{array}\right) .
$$

The potential operator for $J=0$ vanishes identically:

$$
\mathcal{O}^{(0)}=\sum_{i k r s=-1 / 2}^{1 / 2} \varphi_{i}^{*} \varphi_{r} \varphi_{k}^{*} \varphi_{s} \Sigma_{i k}^{(0), 0} \Sigma_{r s}^{(0), 0}=\frac{1}{2}\left|\varphi_{1 / 2} \varphi_{-1 / 2}-\varphi_{1 / 2} \varphi_{-1 / 2}\right|^{2} \equiv 0,
$$

and only the operator for $J=1$ remains:

$$
\begin{aligned}
\mathcal{O}^{(1)} & =\sum_{i k r s=-1 / 2}^{1 / 2} \sum_{a=-1}^{1} \varphi_{i}^{*} \varphi_{r} \varphi_{k}^{*} \varphi_{s} \Sigma_{i k}^{(1), a} \Sigma_{r s}^{(1), a} \\
& =\left|\varphi_{1 / 2}\right|^{4}+\left|\varphi_{-1 / 2}\right|^{4}+2\left|\varphi_{1 / 2}\right|^{2}\left|\varphi_{-1 / 2}\right|^{2} \equiv\left(\varphi^{\dagger} \varphi\right)^{2} .
\end{aligned}
$$

This is equivalent to the fact that we can, employing the more standard definition of operators, express $\left(\varphi^{\dagger} \sigma^{a} \varphi\right)^{2}$ in terms of $\left(\varphi^{\dagger} \varphi\right)^{2}$, using the Fierz relation $\sigma_{i j}^{a} \sigma_{k l}^{a}=2 \delta_{i l} \delta_{k j}-$ $\delta_{i j} \delta_{k l}$. Here, $\sigma_{i j}^{a}$ are the usual Pauli matrices.

\footnotetext{
${ }^{1}$ We assume the invariance of the Lagrangian under a global U(1) symmetry under which only the scalar fields transform non-trivially, so that we do not introduce additional "exotic" operators for special values of $Y_{\varphi}$ (cf. ref. [8]).
} 


\section{Beta function for a scalar multiplet}

In this section, we present the beta function of the full SM extended by a scalar $\varphi$ furnishing a representation $\left(0, j_{\varphi}, Y_{\varphi}\right)$ under the $\mathrm{SM} \mathrm{SU}(3)_{c} \times \mathrm{SU}(2)_{L} \times \mathrm{U}(1)_{Y}$ gauge group. The Lagrangian we consider is given by

$$
\mathcal{L}=\mathcal{L}_{\varphi}+\mathcal{L}_{\psi}+\mathcal{L}_{H}+\mathcal{L}_{Y}+\mathcal{L}_{\mathrm{QCD}}+\mathcal{L}_{\text {portal }}
$$

where $\mathcal{L}_{\varphi}$ is given in eq. (2.1),

$$
\mathcal{L}_{\mathrm{QCD}}=-\frac{1}{4} G_{\mu \nu}^{A} G^{A \mu \nu}
$$

is the gluonic QCD Lagrangian, and

$\mathcal{L}_{\psi}=\sum_{k} \bar{Q}_{L, k} i \not D Q_{L, k}+\sum_{k} \bar{u}_{R, k} i \not D u_{R, k}+\sum_{k} \bar{d}_{R, k} i \not D d_{R, k}+\sum_{k} \bar{L}_{L, k} i \not D L_{L, k}+\sum_{k} \bar{\ell}_{R, i} i \not D \ell_{R, k}$

are the kinetic terms for the SM fermions, where $Q_{L}$ and $L_{L}$ denote the left-handed quark and lepton doublets, and $u_{R}, d_{R}$, and $\ell_{R}$ the right-handed up-quark, down-quark, and lepton fields. The sums run over the three fermion generations, $k=1,2,3$. Furthermore,

$$
\mathcal{L}_{H}=\left(D_{\mu} H\right)^{\dagger} D^{\mu} H+\mu^{2} H^{\dagger} H-\frac{\lambda_{H}}{4}\left(H^{\dagger} H\right)^{2}
$$

is the Higgs doublet Lagrangian, and the Yukawa Lagrangian is given by

$$
\mathcal{L}_{Y}=-\sum_{k l} \bar{Q}_{L, k} Y_{u}^{k l} H^{c} u_{R, l}-\sum_{k l} \bar{Q}_{L, k} Y_{d}^{k l} H d_{R, l}-\sum_{k l} \bar{L}_{L, k} Y_{\ell}^{k l} H \ell_{R, l}+\text { h.c. },
$$

where $H^{c}=i \sigma_{2} H^{*}$ is the charge-conjugated Higgs field. In this work, we neglect the Yukawa couplings of all light fermions, keeping only the top, bottom, charm, and $\tau$ Yukawas $y_{t}, y_{b}, y_{c}$, and $y_{\tau}$ non-zero. This implies that we can assume the Yukawa matrices to be diagonal and neglect CKM mixing. Finally, the Higgs-portal Lagrangian is given by

$$
\mathcal{L}_{\text {portal }}=-\frac{\lambda_{\varphi H}}{4}\left(\varphi^{\dagger} \varphi\right)\left(H^{\dagger} H\right)-\frac{\lambda_{\varphi H}^{\prime}}{4}\left(\varphi^{\dagger} \tilde{\tau}^{a} \varphi\right)\left(H^{\dagger} \tau^{a} H\right) .
$$

Here, $\tau^{a} \equiv \sigma^{a} / 2$ in terms of the usual Pauli matrices. Note that the second term in eq. (3.6) is absent in the case $j_{\varphi}=0$.

The Lagrangian (3.1) is renormalized in the usual way by introducing field and coupling renormalization constants. For instance, we express the unrenormalized scalar couplings (denoted by the superscript "0") in terms of renormalized couplings as

$$
\lambda_{\varphi}^{(J), 0}=Z_{\lambda_{\varphi}^{(J)}} \lambda_{\varphi}^{(J)}=\left(1+\delta Z_{\lambda_{\varphi}^{(J)}}^{(1)}+\delta Z_{\lambda_{\varphi}^{(J)}}^{(2)}+\ldots\right) \lambda_{\varphi}^{(J)}
$$

and similarly for all other couplings and fields. The superscripts (1) and (2) denote the oneand two-loop contributions, respectively. The ellipsis stands for higher-order terms. We extract the beta function in the $\overline{\mathrm{MS}}$ scheme from the $1 / \epsilon$ poles of the coupling counterterms, as explained in appendix A. We employ dimensional regularization in $d=4-2 \epsilon$ space-time dimensions, and we can treat all particles as massless in our calculation. 


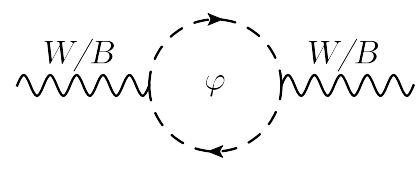

(a)

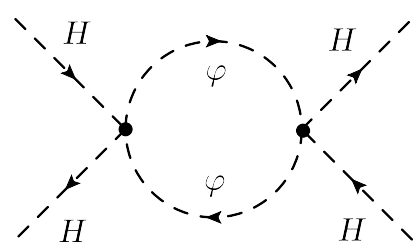

(b)

Figure 1. Feynman diagrams corresponding to the contributions from the scalar field $\varphi$ to the one-loop standard model beta function. Figure (a) shows the contribution to the gauge boson field counterterms which must be subtracted when gauge bosons appear in external states in Green's functions. Figure (b) shows the $\varphi$ loop contributing to the one-loop Higgs quartic coupling beta function.
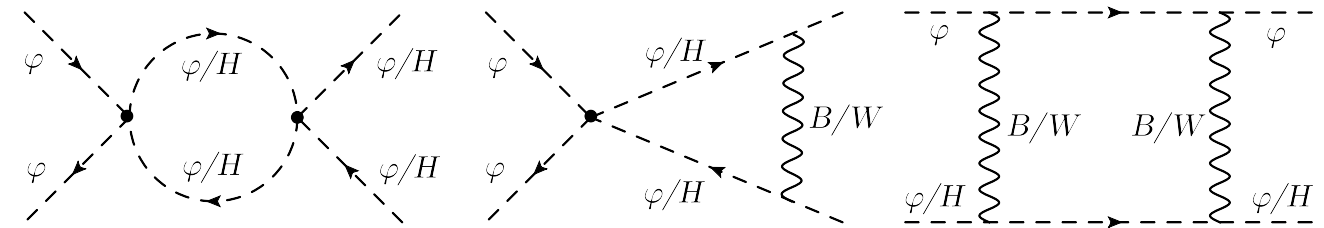

Figure 2. Sample one-loop Feynman diagrams for the calculation of the quartic scalar coupling and the Higgs-portal couplings.

We determine all renormalization constants by calculating the divergent parts of Green's functions with suitably chosen external states (sample Feynman diagrams are shown in figures 1-4). In the calculation of the coupling counterterms, it is necessary to subtract field counterterms corresponding to the external fields. For this reason, all field renormalization constants are calculated in addition to the coupling renormalization constants (the results are collected in appendix B).

In order to isolate the ultraviolet poles, we employ the infrared (IR) rearrangement described in ref. [20], to which we refer for more details. In short, the method amounts to an exact decomposition of all propagators in terms of propagators with a common IR regulator mass, which we call $M_{\text {IRA }}$. Effectively, we introduce a common mass $M_{\text {IRA }}$ for the scalar, the gauge-boson, and the ghost fields,

$$
\mathcal{L}_{\mathrm{IRA}}=\frac{1}{2} M_{\mathrm{IRA}}^{2} W_{\mu}^{a} W^{a \mu}+\frac{1}{2} M_{\mathrm{IRA}}^{2} B_{\mu} B^{\mu}-M_{\mathrm{IRA}}^{2} \varphi_{i}^{\dagger} \varphi_{i}-M_{\mathrm{IRA}}^{2} \bar{u}_{W}^{a} u_{W}^{a} .
$$

These masses get renormalized at higher orders, and we introduce corresponding mass counterterms $Z_{M_{\mathrm{IRA}}, i}, i=W, B, \varphi$, in the usual way $\left(M_{\text {bare }}^{2}=Z_{M^{2}} M^{2}\right)$. The explicit results needed for our work are collected in appendix B. We explicitly verified that all our results are independent of the regulator mass $M_{\text {IRA }}$, as it should be.

All $\mathcal{O}(10000)$ Feynman diagrams were calculated using self-written FORM [21] routines, encoding the algorithm presented in ref. [22]. The Feynman diagrams were generated using qgraf [23]. The $\mathrm{SU}(2)$ group algebra and renormalization was performed independently by the two authors; the results are in complete agreement. We describe further analytic checks of our calculation in appendix A. 
The beta functions are defined as the logarithmic derivatives of the couplings with respect to the renormalization scale,

$$
\mu \frac{d}{d \mu} g_{i}=\beta_{g_{i}}
$$

They are given in terms of the coupling counterterms by

$$
\beta_{g_{i}}=g_{i} \sum_{k} a_{k} g_{k} \frac{\partial Z_{g_{i}, 1}}{\partial g_{k}}
$$

for all couplings, denoted here collectively by $g_{i}=g_{1}, g_{2}, g_{s}, \lambda_{\varphi}^{(J)}, \lambda_{\varphi H}, \lambda_{\varphi H}^{\prime}, \lambda_{H}, y_{t}, y_{b}, y_{c}, y_{\tau}$. Here, $Z_{g_{i}, 1}$ is the residue of the $1 / \epsilon$ pole of the counterterm and $a_{k}=1$ when $g_{k}$ is a gauge or Yukawa coupling while $a_{k}=2$ when $g_{k}$ is a quartic scalar coupling. Expanding the beta function by loop order as $\beta_{g_{i}}=\beta_{g_{i}}^{(1)}+\beta_{g_{i}}^{(2)}+\ldots$, we find for the one-loop contributions

$$
\begin{aligned}
\beta_{g_{1}}^{(1)}= & \frac{g_{1}^{3}}{16 \pi^{2}}\left(\frac{Y_{\varphi}^{2}}{12} \mathcal{D}\left(j_{\varphi}\right)+\frac{1}{6}+\frac{20}{9} n_{g}\right) \\
\beta_{g_{2}}^{(1)}= & \frac{g_{2}^{3}}{16 \pi^{2}}\left(\frac{1}{9} \mathcal{J}\left(j_{\varphi}\right) \mathcal{D}\left(j_{\varphi}\right)-\frac{43}{6}+\frac{4}{3} n_{g}\right), \\
\beta_{g_{s}}^{(1)}= & \frac{g_{s}^{3}}{16 \pi^{2}}\left(\frac{4}{3} n_{g}-11\right) \\
\beta_{y_{t}}^{(1)}= & \frac{y_{t}}{16 \pi^{2}}\left(-\frac{17 g_{1}^{2}}{12}-\frac{9 g_{2}^{2}}{4}-8 g_{s}^{2}+\frac{9 y_{t}^{2}}{2}+3 y_{c}^{2}+\frac{3 y_{b}^{2}}{2}+y_{\tau}^{2}\right), \\
\beta_{y_{b}}^{(1)}= & \frac{y_{b}}{16 \pi^{2}}\left(-\frac{5 g_{1}^{2}}{12}-\frac{9 g_{2}^{2}}{4}-8 g_{s}^{2}+\frac{3 y_{t}^{2}}{2}+3 y_{c}^{2}+\frac{9 y_{b}^{2}}{2}+y_{\tau}^{2}\right), \\
\beta_{y_{c}}^{(1)}= & \frac{y_{c}}{16 \pi^{2}}\left(-\frac{17 g_{1}^{2}}{12}-\frac{9 g_{2}^{2}}{4}-8 g_{s}^{2}+3 y_{t}^{2}+\frac{9 y_{c}^{2}}{2}+3 y_{b}^{2}+y_{\tau}^{2}\right), \\
\beta_{y_{\tau}}^{(1)}= & \frac{y_{\tau}}{16 \pi^{2}}\left(-\frac{15 g_{1}^{2}}{4}-\frac{9 g_{2}^{2}}{4}+3 y_{t}^{2}+3 y_{c}^{2}+3 y_{b}^{2}+\frac{5 y_{\tau}^{2}}{2}\right), \\
\beta_{\lambda_{H}}^{(1)}= & \frac{g_{1}^{2}}{16 \pi^{2}}\left(\frac{3 g_{1}^{2}}{2}-3 \lambda_{H}\right)+\frac{g_{2}^{2}}{16 \pi^{2}}\left(\frac{9 g_{2}^{2}}{2}-9 \lambda_{H}\right)+\frac{3 g_{1}^{2} g_{2}^{2}}{16 \pi^{2}} \\
& -\frac{1}{2 \pi^{2}}\left(3 y_{t}^{4}+3 y_{c}^{4}+3 y_{b}^{4}+y_{\tau}^{4}\right)+\frac{\lambda_{H}}{4 \pi^{2}}\left(3 y_{t}^{2}+3 y_{c}^{2}+3 y_{b}^{2}+y_{\tau}^{2}\right) \\
& +\frac{3 \lambda_{H}^{2}}{8 \pi^{2}}+\frac{\lambda_{\varphi H}^{2}}{64 \pi^{2}} \mathcal{D}(j \varphi)+\frac{\lambda_{\varphi H}^{\prime 2}}{768 \pi^{2}} \mathcal{D}(j \varphi) \mathcal{J}(j \varphi), \\
\beta_{\lambda_{\varphi H}}^{(1)}= & \frac{g_{1}^{2}}{16 \pi^{2}}\left[3 g_{1}^{2} Y_{\varphi}^{2}-\frac{3 \lambda_{\varphi H}}{2}\left(1+Y_{\varphi}^{2}\right)\right]+\frac{g_{2}^{2}}{16 \pi^{2}}\left[12 g_{2}^{2} \mathcal{J}(j \varphi)-\lambda_{\varphi H}\left(\frac{9}{2}+6 \mathcal{J}(j \varphi)\right)\right] \\
& +\frac{\lambda_{\varphi H}}{8 \pi^{2}}\left(3 y_{t}^{2}+3 y_{c}^{2}+3 y_{b}^{2}+y_{\tau}^{2}\right)+\frac{3 \lambda_{H} \lambda_{\varphi H}}{16 \pi^{2}}+\frac{\lambda_{\varphi H}^{2}}{16 \pi^{2}} \\
& +\frac{\lambda_{\varphi}^{\prime 2}}{64 \pi^{2}} \mathcal{J}(j \varphi)+\frac{\lambda_{\varphi H}}{8 \pi^{2}} \sum_{J}^{\prime} \lambda_{\varphi}^{(J)} \frac{\mathcal{D}(J)}{\mathcal{D}(j \varphi)}, \\
&
\end{aligned}
$$




$$
\begin{aligned}
\beta_{\lambda_{\varphi H}^{\prime}}^{(1)}= & -\frac{3 g_{1}^{2} \lambda_{\varphi H}^{\prime}}{32 \pi^{2}}\left(1+Y_{\varphi}^{2}\right)-\frac{g_{2}^{2} \lambda_{\varphi H}^{\prime}}{16 \pi^{2}}\left(\frac{9}{2}+6 \mathcal{J}\left(j_{\varphi}\right)\right)+\frac{3 g_{1}^{2} g_{2}^{2}}{2 \pi^{2}} Y_{\varphi} \\
& +\frac{\lambda_{\varphi H}^{\prime}}{8 \pi^{2}}\left(3 y_{t}^{2}+3 y_{c}^{2}+3 y_{b}^{2}+y_{\tau}^{2}\right)+\frac{\lambda_{\varphi H}^{\prime} \lambda_{H}}{16 \pi^{2}}+\frac{\lambda_{\varphi H}^{\prime} \lambda_{\varphi H}}{8 \pi^{2}} \\
& +\frac{\lambda_{\varphi H}^{\prime}}{16 \pi^{2}} \sum_{J}^{\prime} \lambda_{\varphi}^{(J)} \frac{\mathcal{D}(J)\left(\mathcal{J}(J)-2 \mathcal{J}\left(j_{\varphi}\right)\right)}{\mathcal{J}\left(j_{\varphi}\right) \mathcal{D}\left(j_{\varphi}\right)}, \\
\beta_{\lambda_{\varphi}^{(J)}}^{(1)}= & \left(\frac{\lambda_{\varphi}^{(J)}}{4 \pi}\right)^{2}+\frac{3 g_{2}^{2}}{8 \pi^{2}}\left(g_{2}^{2}\left[\left(\mathcal{J}(J)-2 \mathcal{J}\left(j_{\varphi}\right)\right)^{2}+\left(\mathcal{J}(J)-2 \mathcal{J}\left(j_{\varphi}\right)\right)\right]-2 \lambda_{\varphi}^{(J)} \mathcal{J}\left(j_{\varphi}\right)\right) \\
& +\frac{1}{4 \pi^{2}} \sum_{J_{1}, J_{2}}^{\prime} \lambda_{\varphi}^{\left(J_{1}\right)} \lambda_{\varphi}^{\left(J_{2}\right)} K\left(J_{1}, J_{2}, J\right)+\frac{\lambda_{\varphi H}^{2}}{32 \pi^{2}}+\frac{\lambda_{\varphi H}^{\prime 2}}{256 \pi^{2}}\left(\mathcal{J}(J)-2 \mathcal{J}\left(j_{\varphi}\right)\right) \\
& +\frac{3 g_{1}^{4}}{32 \pi^{2}} Y_{\varphi}^{4}-\frac{3 g_{1}^{2} \lambda_{\varphi}^{(J)}}{16 \pi^{2}} Y_{\varphi}^{2}+\frac{3 g_{1}^{2} g_{2}^{2}}{8 \pi^{2}}\left(\mathcal{J}(J)-2 \mathcal{J}\left(j_{\varphi}\right)\right) Y_{\varphi}^{2} .
\end{aligned}
$$

Here and in the following, a prime on the summation sign indicates a restricted sum over indices, defined by

$$
\sum_{J}^{\prime} \ldots \equiv \sum_{J=0}^{2 j_{\varphi}} \frac{1+(-1)^{2 j_{\varphi}-J}}{2} \ldots
$$

The sum effectively runs over even or odd values of $J$ only, if the weak isospin $j_{\varphi}$ of the scalar multiplet is integer or half-integer, respectively. (For instance, for a SU(2) septuplet with $j_{\varphi}=3$ we have $J=0,2,4,6$.) The group-theory functions are defined as $\mathcal{J}\left(j_{\varphi}\right) \equiv j_{\varphi}\left(j_{\varphi}+1\right), \mathcal{D}\left(j_{\varphi}\right) \equiv 2 j_{\varphi}+1$, and

$$
K\left(J_{1}, J_{2}, J_{3}\right) \equiv \mathcal{D}\left(J_{1}\right) \mathcal{D}\left(J_{2}\right)\left\{\begin{array}{lll}
J_{1} & j_{\varphi} & j_{\varphi} \\
j_{\varphi} & J_{2} & j_{\varphi} \\
j_{\varphi} & j_{\varphi} & J_{3}
\end{array}\right\}
$$

in terms of the Wigner $9 j$ symbol [24] - see section 4 for more details. Moreover, $n_{g}=3$ denotes the number of SM fermion generations. Our one-loop results for the pure SM contributions agree with those in ref. [11]. The scalar contribution to $\beta_{g_{2}}^{(1)}$ agrees with the expression given in ref. [8]. The remaining results are new.

We note here that, at one-loop, the only beta functions which receive contributions from the complex scalar are the gauge and quartic scalar couplings. The contributions to the gauge coupling beta functions arise in our calculation from the gauge boson field counterterms (figure 1a). In addition to SM terms, the Higgs quartic coupling beta function gains two terms from diagrams with scalar loops, shown in figure $1 \mathrm{~b}$.

The beta functions for the Higgs-portal couplings and quartic scalar couplings are subdivided into three classes: scalar only terms, mixed scalar-gauge terms, and gauge-only terms. Sample diagrams of each of these classes are shown in figure 2. The Higgs-portal coupling beta functions also receive contributions from Yukawa couplings, coming from the field counterms for the external Higgs fields in the four-point Green's functions. 


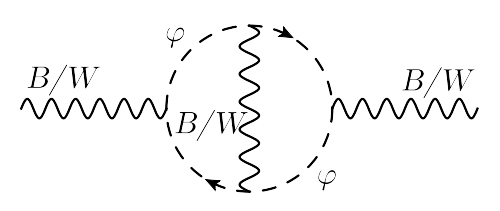

(a)
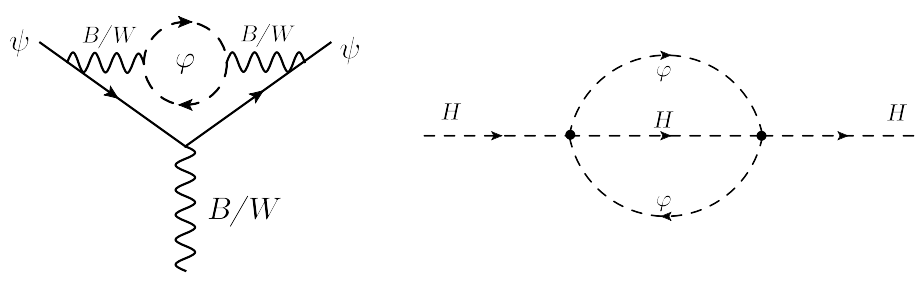

(b)

Figure 3. Sample Feynman diagrams showing two-loop contributions from the scalar field $\varphi$ to gauge and Yukawa coupling beta functions. Figure (a) shows contributions to gauge coupling beta functions from gauge boson self-energies as well as $\varphi$ insertions to the three-point Green's function with a single external gauge boson. Here, $\psi$ represents any fermion which couples to the gauge fields. Figure (b) shows a diagram from the two-loop Higgs self-energy which contributes to the Yukawa coupling beta functions.

In order to express these contributions in terms of the operators in the scalar potential (2.14), we rewrite all SU(2) generators appearing in the $W$-boson vertices in terms of the Sigma matrices defined in eq. (2.13), and use completeness relations for the ClebschGordan coefficients to simplify the terms. A similar strategy is applied for the "mixed" contributions involving both gauge and scalar interactions. The detailed relations that we use are discussed in section 4. In several cases, particular care has to be taken, as the sum over indices in the completeness relations runs over all possible values of the $J$ spin quantum number, while the local scalar interactions can only involve the restricted sums over odd or even values. Gauge invariance ensures that the final result can be expressed in terms of restricted sums only.

A comment on our treatment of $\gamma_{5}$ is in order. Diagrams containing fermion triangles can contribute terms with an odd number of $\gamma_{5}$ matrices to the gauge-boson field counterterm and gauge coupling counterterms. We took the corresponding contributions to the gauge coupling beta functions from the literature [9, 11], and calculated only the additional scalar contributions at one- and two-loop (sample Feynman diagrams showing these contributions are given in figure 3a). For all other (scalar and Yukawa) beta functions, we performed the two-loop calculation including also the full set of SM particles. We verified explicitly that, in our calculation, only traces with an even number of $\gamma_{5}$ matrices in closed fermion loops appeared. According to common lore [25], we evaluated these traces using naive anticommuting $\gamma_{5}$. We find the following two-loop results:

$$
\begin{aligned}
\beta_{g_{1}}^{(2)}= & \frac{g_{1}^{5}}{\left(16 \pi^{2}\right)^{2}}\left(\frac{Y_{\varphi}^{4}}{4} \mathcal{D}\left(j_{\varphi}\right)+\frac{95}{27} n_{g}+\frac{1}{2}\right) \\
& +\frac{g_{1}^{3} g_{2}^{2}}{\left(16 \pi^{2}\right)^{2}}\left(Y_{\varphi}^{2} \mathcal{D}\left(j_{\varphi}\right) \mathcal{J}\left(j_{\varphi}\right)+n_{g}+\frac{3}{2}\right)+\frac{g_{1}^{3} g_{s}^{2}}{\left(16 \pi^{2}\right)^{2}} \frac{44}{9} n_{g} \\
& -\frac{g_{1}^{3}}{\left(16 \pi^{2}\right)^{2}}\left(\frac{17}{6}\left(y_{t}^{2}+y_{c}^{2}\right)+\frac{5}{6} y_{b}^{2}+\frac{5}{2} y_{\tau}^{2}\right),
\end{aligned}
$$




$$
\begin{aligned}
& \beta_{g_{2}}^{(2)}=\frac{g_{2}^{5}}{\left(16 \pi^{2}\right)^{2}}\left(\frac{4}{3} \mathcal{D}\left(j_{\varphi}\right) \mathcal{J}\left(j_{\varphi}\right)^{2}+\frac{4}{9} \mathcal{D}\left(j_{\varphi}\right) \mathcal{J}\left(j_{\varphi}\right)-\frac{136}{3}+\frac{49}{3} n_{g}+\frac{13}{6}\right) \\
& +\frac{g_{1}^{2} g_{2}^{3}}{\left(16 \pi^{2}\right)^{2}}\left(\frac{Y_{\varphi}^{2}}{3} \mathcal{D}\left(j_{\varphi}\right) \mathcal{J}\left(j_{\varphi}\right)+\frac{1}{3} n_{g}+\frac{1}{2}\right)+\frac{g_{s}^{2} g_{2}^{3}}{\left(16 \pi^{2}\right)^{2}} 4 n_{g} \\
& -\frac{g_{2}^{3}}{\left(16 \pi^{2}\right)^{2}}\left(\frac{3}{2}\left(y_{t}^{2}+y_{c}^{2}+y_{b}^{2}\right)+\frac{1}{2} y_{\tau}^{2}\right) \text {, } \\
& \beta_{g_{s}}^{(2)}=\frac{g_{s}^{5}}{\left(16 \pi^{2}\right)^{2}}\left(\frac{76}{3} n_{g}-102\right)+\frac{g_{1}^{2} g_{s}^{3}}{\left(16 \pi^{2}\right)^{2}} \frac{11}{18} n_{g}+\frac{g_{2}^{2} g_{s}^{3}}{\left(16 \pi^{2}\right)^{2}} \frac{3}{2} n_{g} \\
& -2 \frac{g_{s}^{3}}{\left(16 \pi^{2}\right)^{2}}\left(y_{t}^{2}+y_{c}^{2}+y_{b}^{2}\right) \text {, } \\
& \beta_{y_{t}}^{(2)}=\frac{y_{t} g_{1}^{4}}{\left(16 \pi^{2}\right)^{2}}\left(\frac{1}{8}+\frac{145}{81} n_{g}+\frac{5}{27} \mathcal{D}\left(j_{\varphi}\right) Y_{\varphi}^{2}\right) \\
& +\frac{y_{t} g_{2}^{4}}{\left(16 \pi^{2}\right)^{2}}\left(n_{g}+\frac{1}{3} \mathcal{J}\left(j_{\varphi}\right) \mathcal{D}\left(j_{\varphi}\right)-\frac{35}{4}\right)-\frac{3}{4} \frac{y_{t} g_{1}^{2} g_{2}^{2}}{\left(16 \pi^{2}\right)^{2}} \\
& +\frac{y_{t} g_{s}^{4}}{\left(16 \pi^{2}\right)^{2}}\left(\frac{80}{9} n_{g}-\frac{404}{3}\right) \\
& +\frac{y_{t} g_{1}^{2}}{\left(16 \pi^{2}\right)^{2}}\left(\frac{131}{16} y_{t}^{2}+\frac{7}{48} y_{b}^{2}+\frac{85}{24} y_{c}^{2}+\frac{25}{8} y_{\tau}^{2}\right)+\frac{19}{9} \frac{y_{t} g_{1}^{2} g_{s}^{2}}{\left(16 \pi^{2}\right)^{2}} \\
& +\frac{y_{t} g_{2}^{2}}{\left(16 \pi^{2}\right)^{2}}\left(\frac{225}{16} y_{t}^{2}+\frac{99}{16} y_{b}^{2}+\frac{45}{8} y_{c}^{2}+\frac{15}{8} y_{\tau}^{2}\right)+9 \frac{y_{t} g_{2}^{2} g_{s}^{2}}{\left(16 \pi^{2}\right)^{2}} \\
& +\frac{y_{t} g_{s}^{2}}{\left(16 \pi^{2}\right)^{2}}\left(36 y_{t}^{2}+4 y_{b}^{2}+20 y_{c}^{2}\right)-\frac{y_{t}^{3}}{\left(16 \pi^{2}\right)^{2}}\left(12 y_{t}^{2}+\frac{11}{4} y_{b}^{2}+\frac{27}{4} y_{c}^{2}+\frac{9}{4} y_{\tau}^{2}+3 \lambda_{H}\right) \\
& +\frac{y_{t}}{\left(16 \pi^{2}\right)^{2}}\left(\frac{1}{32} \mathcal{D}\left(j_{\varphi}\right) \lambda_{\varphi H}^{2}+\frac{1}{128} \mathcal{J}\left(j_{\varphi}\right) \mathcal{D}\left(j_{\varphi}\right)\left(\lambda_{\varphi H}^{\prime}\right)^{2}+\frac{3}{8} \lambda_{H}^{2}\right) \\
& -\frac{y_{t}}{\left(16 \pi^{2}\right)^{2}}\left(\frac{1}{4} y_{b}^{4}+\frac{27}{4} y_{c}^{4}+\frac{9}{4} y_{\tau}^{4}-\frac{15}{4} y_{b}^{2} y_{c}^{2}-\frac{5}{4} y_{b}^{2} y_{\tau}^{2}\right) \text {, } \\
& \beta_{y_{b}}^{(2)}=-\frac{y_{b} g_{1}^{4}}{\left(16 \pi^{2}\right)^{2}}\left(\frac{29}{72}+\frac{5}{81} n_{g}-\frac{7}{216} \mathcal{D}\left(j_{\varphi}\right) Y_{\varphi}^{2}\right) \\
& +\frac{y_{b} g_{2}^{4}}{\left(16 \pi^{2}\right)^{2}}\left(n_{g}+\frac{1}{3} \mathcal{J}\left(j_{\varphi}\right) \mathcal{D}\left(j_{\varphi}\right)-\frac{35}{4}\right)-\frac{9}{4} \frac{y_{b} g_{1}^{2} g_{2}^{2}}{\left(16 \pi^{2}\right)^{2}} \\
& +\frac{y_{b} g_{s}^{4}}{\left(16 \pi^{2}\right)^{2}}\left(\frac{80}{9} n_{g}-\frac{404}{3}\right) \\
& +\frac{y_{b} g_{1}^{2}}{\left(16 \pi^{2}\right)^{2}}\left(\frac{91}{48} y_{t}^{2}+\frac{79}{16} y_{b}^{2}+\frac{85}{24} y_{c}^{2}+\frac{25}{8} y_{\tau}^{2}\right)+\frac{31}{9} \frac{y_{b} g_{1}^{2} g_{s}^{2}}{\left(16 \pi^{2}\right)^{2}} \\
& +\frac{y_{b} g_{2}^{2}}{\left(16 \pi^{2}\right)^{2}}\left(\frac{99}{16} y_{t}^{2}+\frac{225}{16} y_{b}^{2}+\frac{45}{8} y_{c}^{2}+\frac{15}{8} y_{\tau}^{2}\right)+9 \frac{y_{b} g_{2}^{2} g_{s}^{2}}{\left(16 \pi^{2}\right)^{2}} \\
& +\frac{y_{b} g_{s}^{2}}{\left(16 \pi^{2}\right)^{2}}\left(4 y_{t}^{2}+36 y_{b}^{2}+20 y_{c}^{2}\right)-\frac{y_{b}^{3}}{\left(16 \pi^{2}\right)^{2}}\left(\frac{11}{4} y_{t}^{2}+12 y_{b}^{2}+\frac{27}{4} y_{c}^{2}+\frac{9}{4} y_{\tau}^{2}+3 \lambda_{H}\right) \\
& +\frac{y_{b}}{\left(16 \pi^{2}\right)^{2}}\left(\frac{1}{32} \mathcal{D}\left(j_{\varphi}\right) \lambda_{\varphi H}^{2}+\frac{1}{128} \mathcal{J}\left(j_{\varphi}\right) \mathcal{D}\left(j_{\varphi}\right)\left(\lambda_{\varphi H}^{\prime}\right)^{2}+\frac{3}{8} \lambda_{H}^{2}\right) \\
& -\frac{y_{b}}{\left(16 \pi^{2}\right)^{2}}\left(\frac{1}{4} y_{t}^{4}+\frac{27}{4} y_{c}^{4}+\frac{9}{4} y_{\tau}^{4}-\frac{15}{4} y_{t}^{2} y_{c}^{2}-\frac{5}{4} y_{t}^{2} y_{\tau}^{2}\right),
\end{aligned}
$$




$$
\begin{aligned}
& \beta_{y_{c}}^{(2)}=\frac{y_{c} g_{1}^{4}}{\left(16 \pi^{2}\right)^{2}}\left(\frac{1}{8}+\frac{145}{81} n_{g}+\frac{5}{27} \mathcal{D}\left(j_{\varphi}\right) Y_{\varphi}^{2}\right) \\
& +\frac{y_{c} g_{2}^{4}}{\left(16 \pi^{2}\right)^{2}}\left(n_{g}+\frac{1}{3} \mathcal{J}\left(j_{\varphi}\right) \mathcal{D}\left(j_{\varphi}\right)-\frac{35}{4}\right)-\frac{3}{4} \frac{y_{c} g_{1}^{2} g_{2}^{2}}{\left(16 \pi^{2}\right)^{2}} \\
& +\frac{y_{c} g_{s}^{4}}{\left(16 \pi^{2}\right)^{2}}\left(\frac{80}{9} n_{g}-\frac{404}{3}\right) \\
& +\frac{y_{c} g_{1}^{2}}{\left(16 \pi^{2}\right)^{2}}\left(\frac{85}{24} y_{t}^{2}+\frac{25}{24} y_{b}^{2}+\frac{131}{16} y_{c}^{2}+\frac{25}{8} y_{\tau}^{2}\right)+\frac{19}{9} \frac{y_{c} g_{1}^{2} g_{s}^{2}}{\left(16 \pi^{2}\right)^{2}} \\
& +\frac{y_{c} g_{2}^{2}}{\left(16 \pi^{2}\right)^{2}}\left(\frac{45}{8} y_{t}^{2}+\frac{45}{8} y_{b}^{2}+\frac{225}{16} y_{c}^{2}+\frac{15}{8} y_{\tau}^{2}\right)+9 \frac{y_{c} g_{2}^{2} g_{s}^{2}}{\left(16 \pi^{2}\right)^{2}} \\
& +\frac{y_{c} g_{s}^{2}}{\left(16 \pi^{2}\right)^{2}}\left(20 y_{t}^{2}+20 y_{b}^{2}+36 y_{c}^{2}\right)-\frac{y_{c}^{3}}{\left(16 \pi^{2}\right)^{2}}\left(\frac{27}{4} y_{t}^{2}+\frac{27}{4} y_{b}^{2}+12 y_{c}^{2}+\frac{9}{4} y_{\tau}^{2}+3 \lambda_{H}\right) \\
& +\frac{y_{c}}{\left(16 \pi^{2}\right)^{2}}\left(\frac{1}{32} \mathcal{D}\left(j_{\varphi}\right) \lambda_{\varphi H}^{2}+\frac{1}{128} \mathcal{J}\left(j_{\varphi}\right) \mathcal{D}\left(j_{\varphi}\right)\left(\lambda_{\varphi H}^{\prime}\right)^{2}+\frac{3}{8} \lambda_{H}^{2}\right) \\
& -\frac{y_{c}}{\left(16 \pi^{2}\right)^{2}}\left(\frac{27}{4} y_{t}^{4}+\frac{27}{4} y_{b}^{4}+\frac{9}{4} y_{\tau}^{4}-\frac{3}{2} y_{t}^{2} y_{b}^{2}\right) \text {, } \\
& \beta_{y_{\tau}}^{(2)}=\frac{y_{\tau} g_{1}^{4}}{\left(16 \pi^{2}\right)^{2}}\left(\frac{17}{24}+\frac{55}{9} n_{g}+\frac{13}{24} \mathcal{D}\left(j_{\varphi}\right) Y_{\varphi}^{2}\right) \\
& +\frac{y_{\tau} g_{2}^{4}}{\left(16 \pi^{2}\right)^{2}}\left(n_{g}+\frac{1}{3} \mathcal{J}\left(j_{\varphi}\right) \mathcal{D}\left(j_{\varphi}\right)-\frac{35}{4}\right)+\frac{9}{4} \frac{y_{\tau} g_{1}^{2} g_{2}^{2}}{\left(16 \pi^{2}\right)^{2}} \\
& +\frac{y_{\tau} g_{1}^{2}}{\left(16 \pi^{2}\right)^{2}}\left(\frac{85}{24} y_{t}^{2}+\frac{25}{24} y_{b}^{2}+\frac{85}{24} y_{c}^{2}+\frac{179}{16} y_{\tau}^{2}\right) \\
& +\frac{y_{\tau} g_{2}^{2}}{\left(16 \pi^{2}\right)^{2}}\left(\frac{45}{8} y_{t}^{2}+\frac{45}{8} y_{b}^{2}+\frac{45}{8} y_{c}^{2}+\frac{165}{16} y_{\tau}^{2}\right) \\
& +\frac{y_{\tau} g_{s}^{2}}{\left(16 \pi^{2}\right)^{2}}\left(20 y_{t}^{2}+20 y_{b}^{2}+20 y_{c}^{2}\right)-\frac{y_{\tau}^{3}}{\left(16 \pi^{2}\right)^{2}}\left(\frac{27}{4} y_{t}^{2}+\frac{27}{4} y_{b}^{2}+\frac{27}{4} y_{c}^{2}+3 y_{\tau}^{2}+3 \lambda_{H}\right) \\
& +\frac{y_{\tau}}{\left(16 \pi^{2}\right)^{2}}\left(\frac{1}{32} \mathcal{D}\left(j_{\varphi}\right) \lambda_{\varphi H}^{2}+\frac{1}{128} \mathcal{J}\left(j_{\varphi}\right) \mathcal{D}\left(j_{\varphi}\right)\left(\lambda_{\varphi H}^{\prime}\right)^{2}+\frac{3}{8} \lambda_{H}^{2}\right) \\
& -\frac{y_{\tau}}{\left(16 \pi^{2}\right)^{2}}\left(\frac{27}{4} y_{t}^{4}+\frac{27}{4} y_{b}^{4}+\frac{27}{4} y_{c}^{4}-\frac{3}{2} y_{t}^{2} y_{b}^{2}\right), \\
& \beta_{k}^{(2)}=\frac{1}{\left(16 \pi^{2}\right)^{2}}\left(g_{1}^{6} B_{k, 60}+g_{1}^{4} g_{2}^{2} B_{k, 42}+g_{2}^{2} g_{2}^{4} B_{k, 24}+g_{2}^{6} B_{k, 06}+B_{k, 00}\right. \\
& \left.+g_{1}^{4} B_{k, 40}+g_{1}^{2} g_{2}^{2} B_{k, 22}+g_{2}^{4} B_{k, 04}+g_{1}^{2} B_{k, 20}+g_{2}^{2} B_{k, 02}\right),
\end{aligned}
$$

where the coefficients are given, for $k=\lambda_{\varphi}^{(J)}, \lambda_{\varphi H}, \lambda_{\varphi H}^{\prime}, \lambda_{H}$, by

$$
\begin{aligned}
B_{\varphi, 60}^{(J)}= & -\frac{Y_{\varphi}^{6}}{4}\left(\frac{7}{3} \mathcal{D}\left(j_{\varphi}\right)+15\right)-Y_{\varphi}^{4}\left(\frac{7}{6}+\frac{80}{9} n_{g}\right) \\
B_{\varphi, 42}^{(J)}= & Y_{\varphi}^{4}\left[\mathcal{J}\left(j_{\varphi}\right)\left(\frac{7}{3} \mathcal{D}\left(j_{\varphi}\right)+15\right)-\mathcal{J}(J)\left(\frac{7}{6} \mathcal{D}\left(j_{\varphi}\right)+15\right)\right] \\
& -Y_{\varphi}^{2}\left(\frac{7}{3}+\frac{160}{9} n_{g}\right)\left(\mathcal{J}(J)-2 \mathcal{J}\left(j_{\varphi}\right)\right)
\end{aligned}
$$




$$
\begin{aligned}
& B_{\varphi, 24}^{(J)}=Y_{\varphi}^{2}\left[\mathcal{J}\left(j_{\varphi}\right)\left(60 \mathcal{J}\left(j_{\varphi}\right)+\frac{28}{9} \mathcal{D}\left(j_{\varphi}\right) \mathcal{J}\left(j_{\varphi}\right)-\frac{218}{3}+\frac{64}{3} n_{g}\right)\right. \\
& \left.-\mathcal{J}(J)\left(15 \mathcal{J}(J)+\frac{14}{9} \mathcal{D}\left(j_{\varphi}\right) \mathcal{J}\left(j_{\varphi}\right)-\frac{109}{3}+\frac{32}{3} n_{g}\right)\right], \\
& B_{\varphi, 06}^{(J)}=\left(\frac{\mathcal{J}(J)}{2}-\mathcal{J}\left(j_{\varphi}\right)\right)\left(\frac{\mathcal{J}(J)}{2}-\mathcal{J}\left(j_{\varphi}\right)+\frac{1}{2}\right) \\
& \times\left(\frac{584}{3}-\frac{112}{9} \mathcal{J}\left(j_{\varphi}\right) \mathcal{D}\left(j_{\varphi}\right)-240 \mathcal{J}\left(j_{\varphi}\right)-\frac{256}{3} n_{g}\right)+216 \mathcal{J}\left(j_{\varphi}\right)^{2}, \\
& B_{\varphi, 20}^{(J)}=Y_{\varphi}^{2}\left(8 \sum_{J_{1}, J_{2}}^{\prime} K\left(J_{1}, J_{2}, J\right) \lambda_{\varphi}^{\left(J_{1}\right)} \lambda_{\varphi}^{\left(J_{2}\right)}-\left(\lambda_{\varphi}^{(J)}\right)^{2}\right) \\
& +\frac{1}{8}\left(\lambda_{\varphi H}^{\prime}\right)^{2}\left(\mathcal{J}(J)-2 \mathcal{J}\left(j_{\varphi}\right)\right)+\lambda_{\varphi H}^{2}, \\
& B_{\varphi, 02}^{(J)}=\left(\lambda_{\varphi}^{(J)}\right)^{2}\left(8 \mathcal{J}\left(j_{\varphi}\right)-3 \mathcal{J}(J)\right)-16 \mathcal{J}\left(j_{\varphi}\right) \sum_{J_{1}, J_{2}}^{\prime} K\left(J_{1}, J_{2}, J\right) \lambda_{\varphi}^{\left(J_{1}\right)} \lambda_{\varphi}^{\left(J_{2}\right)} \\
& +12 \sum_{J_{1}, J_{2}}^{\prime} \sum_{J_{3}, J_{4}}(-1)^{J_{4}-2 j} \lambda_{\varphi}^{\left(J_{1}\right)} \lambda_{\varphi}^{\left(J_{2}\right)} \mathcal{J}\left(J_{4}\right) K\left(J_{1}, J_{2}, J_{3}\right) K\left(J_{3}, J_{4}, J\right)+3 \lambda_{\varphi H}^{2}, \\
& B_{\varphi, 40}^{(J)}=Y_{\varphi}^{4}\left(\frac{9}{8} \lambda_{\varphi}^{(J)}+\frac{11 \mathcal{D}\left(j_{\varphi}\right)}{24} \lambda_{\varphi}^{(J)}+5 \sum_{J_{1}}^{\prime} \lambda_{\varphi}^{\left(J_{1}\right)} \frac{\mathcal{D}\left(J_{1}\right)}{\mathcal{D}\left(j_{\varphi}\right)}\right) \\
& +Y_{\varphi}^{2} \lambda_{\varphi}^{(J)}\left(\frac{11}{12}+\frac{50}{9} n_{g}\right)+\frac{5 \lambda_{\varphi H}}{2} Y_{\varphi}^{2}, \\
& B_{\varphi, 22}^{(J)}=Y_{\varphi}^{2} \sum_{J_{1}}^{\prime} \lambda_{\varphi}^{\left(J_{1}\right)} \frac{\mathcal{D}\left(J_{1}\right)}{\mathcal{D}\left(j_{\varphi}\right)}\left[10 \frac{\mathcal{J}\left(J_{1}\right) \mathcal{J}(J)}{\mathcal{J}\left(j_{\varphi}\right)}-20\left(\mathcal{J}\left(J_{1}\right)+\mathcal{J}(J)\right)+40 \mathcal{J}\left(j_{\varphi}\right)\right] \\
& +Y_{\varphi}^{2} \lambda_{\varphi}^{(J)}\left(2 \mathcal{J}(J)+\mathcal{J}\left(j_{\varphi}\right)\right)+\frac{5}{2} Y_{\varphi} \lambda_{\varphi H}^{\prime}\left(\mathcal{J}(J)-2 \mathcal{J}\left(j_{\varphi}\right)\right) \\
& B_{\varphi, 04}^{(J)}=\lambda_{\varphi}^{(J)}\left[\mathcal{J}\left(j_{\varphi}\right)\left(18 \mathcal{J}\left(j_{\varphi}\right)+\frac{22}{9} \mathcal{D}\left(j_{\varphi}\right) \mathcal{J}\left(j_{\varphi}\right)-\frac{275}{3}+\frac{40}{3} n_{g}\right)\right. \\
& \left.+\mathcal{J}(J)\left(\mathcal{J}(J)-4 \mathcal{J}\left(j_{\varphi}\right)+2\right)\right]+10 \lambda_{\varphi H} \mathcal{J}\left(j_{\varphi}\right) \\
& +4 \sum_{J_{1}, J_{2}}^{\prime} \lambda_{\varphi}^{\left(J_{2}\right)} K\left(J_{1}, J_{2}, J\right)\left(\mathcal{J}\left(J_{1}\right)^{2}-4 \mathcal{J}\left(J_{1}\right) \mathcal{J}\left(j_{\varphi}\right)\right) \\
& +\sum_{J_{1}} \sum_{J_{2}}^{\prime} \lambda_{\varphi}^{\left(J_{2}\right)} K\left(J_{1}, J_{2}, J\right)\left(18 \mathcal{J}\left(J_{1}\right)^{2}-72 \mathcal{J}\left(J_{1}\right) \mathcal{J}\left(j_{\varphi}\right)\right) \\
& +\sum_{J_{1}}^{\prime} \frac{\lambda_{\varphi}^{\left(J_{1}\right)}}{\mathcal{D}\left(j_{\varphi}\right)}\left[80 \mathcal{D}\left(J_{1}\right) \mathcal{J}\left(j_{\varphi}\right)^{2}+40 \mathcal{D}\left(J_{1}\right) \mathcal{J}\left(j_{\varphi}\right)\right. \\
& \left.-20 \mathcal{D}\left(J_{1}\right) \mathcal{J}\left(J_{1}\right)-20 \mathcal{D}\left(J_{1}\right) \mathcal{J}(J)+10 \frac{\mathcal{J}(J) \mathcal{J}\left(J_{1}\right) \mathcal{D}\left(J_{1}\right)}{\mathcal{J}\left(j_{\varphi}\right)}\right],
\end{aligned}
$$




$$
\begin{aligned}
B_{\varphi, 00}^{(J)}= & \lambda_{\varphi}^{(J)} \sum_{J_{1}}^{\prime}\left(\lambda_{\varphi}^{\left(J_{1}\right)}\right)^{2} \frac{\mathcal{D}\left(J_{1}\right)}{\mathcal{D}\left(j_{\varphi}\right)}-4 \sum_{J_{1}, J_{2}}^{\prime} K\left(J_{1}, J_{2}, J\right) \lambda_{\varphi}^{\left(J_{1}\right)} \lambda_{\varphi}^{\left(J_{2}\right)}\left(\lambda_{\varphi}^{\left(J_{1}\right)}+\lambda_{\varphi}^{(J)}\right) \\
& -8 \sum_{J_{1}, J_{2}, J_{3}} \sum_{J_{4}}(-1)^{J_{4}-2 j} K\left(J_{1}, J_{2}, J_{4}\right) K\left(J_{4}, J_{3}, J\right) \lambda_{\varphi}^{\left(J_{1}\right)} \lambda_{\varphi}^{\left(J_{2}\right)} \lambda_{\varphi}^{\left(J_{3}\right)} \\
& -\frac{\lambda_{\varphi}^{(J)}\left(\lambda_{\varphi H}^{\prime}\right)^{2}}{16}\left(2 \mathcal{J}(J)-3 \mathcal{J}\left(j_{\varphi}\right)\right)+\frac{\lambda_{\varphi H}\left(\lambda_{\varphi H}^{\prime}\right)^{2}}{8}\left(\mathcal{J}\left(j_{\varphi}\right)-\mathcal{J}(J)\right) \\
& -\frac{\left(\lambda_{\varphi H}^{\prime}\right)^{2}}{8}\left(3 y_{t}^{2}+3 y_{t}^{2}+3 y_{t}^{2}+y_{\tau}^{2}\right)\left(\mathcal{J}(J)-2 \mathcal{J}\left(j_{\varphi}\right)\right) \\
& -\lambda_{\varphi H}^{2}\left(3 y_{t}^{2}+3 y_{b}^{2}+3 y_{c}^{2}+y_{\tau}^{2}+\frac{5}{4} \lambda_{\varphi}^{(J)}\right)-\frac{\lambda_{\varphi H}^{3}}{2}
\end{aligned}
$$

For the Higgs-portal couplings we find

$$
\begin{aligned}
& B_{\varphi H, 60}=-Y_{\varphi}^{4}\left(\frac{7}{6} \mathcal{D}\left(j_{\varphi}\right)+\frac{15}{4}\right)-Y_{\varphi}^{2}\left(\frac{73}{12}+\frac{160}{9} n_{g}\right), \\
& B_{\varphi H, 42}=-Y_{\varphi}^{2}\left(15 \mathcal{J}\left(j_{\varphi}\right)+\frac{45}{4}\right) \text {, } \\
& B_{\varphi H, 24}=-15 \mathcal{J}\left(j_{\varphi}\right)\left(1+Y_{\varphi}^{2}\right) \text {, } \\
& B_{\varphi H, 06}=\mathcal{J}\left(j_{\varphi}\right)\left(\frac{1129}{3}-\frac{128}{3} n_{g}-60 \mathcal{J}\left(j_{\varphi}\right)-\frac{56}{9} \mathcal{J}\left(j_{\varphi}\right) \mathcal{D}\left(j_{\varphi}\right)\right), \\
& B_{\varphi H, 40}=Y_{\varphi}^{2}\left(\frac{15}{2} \lambda_{H}-\left(19 y_{t}^{2}-5 y_{b}^{2}+19 y_{c}^{2}+25 y_{\tau}^{2}\right)+5 \sum_{J_{1}}^{\prime} \lambda_{\varphi}^{\left(J_{1}\right)} \frac{\mathcal{D}\left(J_{1}\right)}{\mathcal{D}\left(j_{\varphi}\right)}\right) \\
& +Y_{\varphi}^{2} \lambda_{\varphi H}\left(\frac{23}{24}+\frac{11}{48} \mathcal{D}\left(j_{\varphi}\right)\right)+Y_{\varphi}^{4} \lambda_{\varphi H}\left(\frac{5}{16}+\frac{71}{48} \mathcal{D}\left(j_{\varphi}\right)\right) \\
& +\lambda_{\varphi H}\left[\frac{157}{48}+\frac{25}{9} n_{g}\left(1+Y_{\varphi}^{2}\right)\right] \\
& B_{\varphi H, 22}=Y_{\varphi} \mathcal{J}\left(j_{\varphi}\right) \lambda_{\varphi H}^{\prime}+\lambda_{\varphi H}\left(\frac{15}{8}+\frac{5}{2} \mathcal{J}\left(j_{\varphi}\right) Y_{\varphi}^{2}\right) \text {, } \\
& B_{\varphi H, 04}=\mathcal{J}\left(j_{\varphi}\right)\left(30 \lambda_{H}-4\left(3 y_{t}^{2}+3 y_{b}^{2}+3 y_{c}^{2}+y_{\tau}^{2}\right)+20 \sum_{J_{1}}^{\prime} \lambda_{\varphi}^{\left(J_{1}\right)} \frac{\mathcal{D}\left(J_{1}\right)}{\mathcal{D}\left(j_{\varphi}\right)}\right) \\
& -\lambda_{\varphi H}\left[\frac{385}{16}-5 n_{g}+\mathcal{J}\left(j_{\varphi}\right)\left(\frac{263}{6}-\frac{20}{3} n_{g}-\frac{11}{12} \mathcal{D}\left(j_{\varphi}\right)\right)-\mathcal{J}\left(j_{\varphi}\right)^{2}\left(5+\frac{71}{9} \mathcal{D}\left(j_{\varphi}\right)\right)\right], \\
& B_{\varphi H, 20}=\frac{\left(\lambda_{\varphi H}^{\prime}\right)^{2}}{16} \mathcal{J}\left(j_{\varphi}\right)\left(1+Y_{\varphi}^{2}\right)+\frac{\lambda_{\varphi H}^{2}}{4}\left(1+Y_{\varphi}^{2}\right)+\frac{\lambda_{\varphi H}}{12}\left(85 y_{t}^{2}+25 y_{b}^{2}+85 y_{c}^{2}+75 y_{\tau}^{2}\right) \\
& +6 \lambda_{\varphi H} \lambda_{H}+4 Y_{\varphi}^{2} \lambda_{\varphi H} \sum_{J_{1}}^{\prime} \lambda_{\varphi}^{\left(J_{1}\right)} \frac{\mathcal{D}\left(J_{1}\right)}{\mathcal{D}\left(j_{\varphi}\right)} \\
& B_{\varphi H, 02}=\frac{\left(\lambda_{\varphi H}^{\prime}\right)^{2}}{16} \mathcal{J}\left(j_{\varphi}\right)\left(15+4 \mathcal{J}\left(j_{\varphi}\right)\right)+\frac{\lambda_{\varphi H}^{2}}{4}\left(3+4 \mathcal{J}\left(j_{\varphi}\right)\right) \\
& +\frac{15 \lambda_{\varphi H}}{4}\left(3 y_{t}^{2}+3 y_{b}^{2}+3 y_{c}^{2}+y_{\tau}^{2}\right) \\
& +18 \lambda_{\varphi H} \lambda_{H}+16 \lambda_{\varphi H} \sum_{J_{1}}^{\prime} \lambda_{\varphi}^{\left(J_{1}\right)} \frac{\mathcal{D}\left(J_{1}\right) \mathcal{J}\left(j_{\varphi}\right)}{\mathcal{D}\left(j_{\varphi}\right)},
\end{aligned}
$$




$$
\begin{aligned}
B_{\varphi H, 00}= & -\left(\frac{\left(\lambda_{\varphi H}^{\prime}\right)^{2}}{4} \mathcal{J}\left(j_{\varphi}\right)+6 \lambda_{\varphi H} \lambda_{H}+\lambda_{\varphi H}^{2}\right)\left(3 y_{t}^{2}+3 y_{b}^{2}+3 y_{c}^{2}+y_{\tau}^{2}\right) \\
& +40 \lambda_{\varphi H} g_{s}^{2}\left(y_{t}^{2}+y_{b}^{2}+y_{c}^{2}\right)-\frac{\lambda_{\varphi H}}{2}\left(27 y_{t}^{4}+27 y_{b}^{4}+27 y_{c}^{4}+9 y_{\tau}^{4}+42 y_{t}^{2} y_{b}^{2}\right) \\
& -\frac{5 \mathcal{J}\left(j_{\varphi}\right)}{8}\left(\lambda_{\varphi H}^{\prime}\right)^{2} \lambda_{H}-\frac{15}{4} \lambda_{\varphi H} \lambda_{H}^{2}-\lambda_{\varphi H}\left(\lambda_{\varphi H}^{\prime}\right)^{2}\left(\frac{13}{32}+\frac{\mathcal{D}\left(j_{\varphi}\right)}{64}\right) \mathcal{J}\left(j_{\varphi}\right) \\
& -\frac{9}{2} \lambda_{\varphi H}^{2} \lambda_{H}-\lambda_{\varphi H}^{3}\left(\frac{5}{8}+\frac{\mathcal{D}\left(j_{\varphi}\right)}{16}\right) \\
& -\frac{5 \lambda_{\varphi H}}{2} \sum_{J_{1}}^{\prime}\left(\lambda_{\varphi}^{\left(J_{1}\right)}\right)^{2} \frac{\mathcal{D}\left(J_{1}\right)}{\mathcal{D}\left(j_{\varphi}\right)}-\frac{\left(\lambda_{\varphi H}^{\prime}\right)^{2}}{4} \sum_{J_{1}}^{\prime} \lambda_{\varphi}^{\left(J_{1}\right)} \frac{\mathcal{D}\left(J_{1}\right)\left(\mathcal{J}\left(J_{1}\right)-\mathcal{J}\left(j_{\varphi}\right)\right)}{\mathcal{D}\left(j_{\varphi}\right)} \\
& -3 \lambda_{\varphi H}^{2} \sum_{J_{1}}^{\prime} \lambda_{\varphi}^{\left(J_{1}\right)} \frac{\mathcal{D}\left(J_{1}\right)}{\mathcal{D}\left(j_{\varphi}\right)}
\end{aligned}
$$




$$
\begin{aligned}
B_{\varphi H, 00}^{\prime}= & -\left(\lambda_{\varphi H}^{\prime} \lambda_{H}+\lambda_{\varphi H} \lambda_{\varphi H}^{\prime}\right)\left(6 y_{t}^{2}+6 y_{b}^{2}+6 y_{c}^{2}+2 y_{\tau}^{2}\right) \\
& +40 \lambda_{\varphi H}^{\prime} g_{s}^{2}\left(y_{t}^{2}+y_{b}^{2}+y_{c}^{2}\right)-\frac{\lambda_{\varphi H}^{\prime}}{2}\left(27 y_{t}^{4}+27 y_{b}^{4}+27 y_{c}^{4}+9 y_{\tau}^{4}-54 y_{t}^{2} y_{b}^{2}\right) \\
& -5 \lambda_{\varphi H} \lambda_{\varphi H}^{\prime} \lambda_{H}-\frac{7}{4} \lambda_{\varphi H}^{\prime} \lambda_{H}^{2}-\lambda_{\varphi H}^{\prime} \lambda_{\varphi H}^{2}\left(\frac{13}{8}+\frac{\mathcal{D}\left(j_{\varphi}\right)}{16}\right) \\
& +\left(\lambda_{\varphi H}^{\prime}\right)^{3}\left(\frac{5 \mathcal{J}\left(j_{\varphi}\right) \mathcal{D}\left(j_{\varphi}\right)}{192}-\frac{5 \mathcal{J}\left(j_{\varphi}\right)}{32}+\frac{3}{16}\right) \\
& -\frac{\lambda_{\varphi H}^{\prime}}{2} \sum_{J_{1}}^{\prime}\left(\lambda_{\varphi}^{\left(J_{1}\right)}\right)^{2}\left(2 \frac{\mathcal{J}\left(J_{1}\right) \mathcal{D}\left(J_{1}\right)}{\mathcal{J}\left(j_{\varphi}\right) \mathcal{D}\left(j_{\varphi}\right)}-3 \frac{\mathcal{D}\left(J_{1}\right)}{\mathcal{D}\left(j_{\varphi}\right)}\right) \\
& -2 \lambda_{\varphi H} \lambda_{\varphi H}^{\prime} \sum_{J_{1}}^{\prime} \lambda_{\varphi}^{\left(J_{1}\right)}\left(\frac{\mathcal{J}\left(J_{1}\right) \mathcal{D}\left(J_{1}\right)}{\mathcal{J}\left(j_{\varphi}\right) \mathcal{D}\left(j_{\varphi}\right)}-\frac{\mathcal{D}\left(J_{1}\right)}{\mathcal{D}\left(j_{\varphi}\right)}\right) .
\end{aligned}
$$

Our results for the quartic Higgs self coupling are

$$
\begin{aligned}
& B_{H, 60}=-\frac{7}{12} Y_{\varphi}^{2} \mathcal{D}\left(j_{\varphi}\right)-\frac{59}{12}-\frac{80}{9} n_{g}, \\
& B_{H, 42}=-\frac{7}{12} Y_{\varphi}^{2} \mathcal{D}\left(j_{\varphi}\right)-\frac{239}{12}-\frac{80}{9} n_{g}, \\
& B_{H, 24}=-\frac{7}{9} \mathcal{J}\left(j_{\varphi}\right) \mathcal{D}\left(j_{\varphi}\right)-\frac{97}{12}-\frac{16}{3} n_{g}, \\
& B_{H, 06}=-\frac{7}{3} \mathcal{J}\left(j_{\varphi}\right) \mathcal{D}\left(j_{\varphi}\right)+\frac{497}{4}-16 n_{g}, \\
& B_{H, 40}=\frac{1}{24} \lambda_{H}\left(11 Y_{\varphi}^{2} \mathcal{D}\left(j_{\varphi}\right)+229\right)+\frac{5}{4} \lambda_{\varphi H} Y_{\varphi}^{2} \mathcal{D}\left(j_{\varphi}\right) \\
& +\frac{50}{9} n_{g} \lambda_{H}-\left(19 y_{t}^{2}-5 y_{b}^{2}+19 y_{c}^{2}+25 y_{\tau}^{2}\right) \text {, } \\
& B_{H, 22}=\frac{39}{4} \lambda_{H}+\frac{5}{6} \lambda_{\varphi H}^{\prime} Y_{\varphi} \mathcal{J}\left(j_{\varphi}\right) \mathcal{D}\left(j_{\varphi}\right)+2\left(21 y_{t}^{2}+9 y_{b}^{2}+21 y_{c}^{2}+11 y_{\tau}^{2}\right) \text {, } \\
& B_{H, 04}=5 \lambda_{\varphi H} \mathcal{J}\left(j_{\varphi}\right) \mathcal{D}\left(j_{\varphi}\right)-3\left(3 y_{t}^{2}+3 y_{b}^{2}+3 y_{c}^{2}+y_{\tau}^{2}\right) \\
& +\lambda_{H}\left(10 n_{g}-\frac{313}{8}+\frac{11}{6} \mathcal{J}\left(j_{\varphi}\right) \mathcal{D}\left(j_{\varphi}\right)\right) \text {, } \\
& B_{H, 20}=9 \lambda_{H}^{2}+\frac{1}{2} \lambda_{\varphi H}^{2} Y_{\varphi}^{2} \mathcal{D}\left(j_{\varphi}\right)+\frac{1}{24}\left(\lambda_{\varphi H}^{\prime}\right)^{2} Y_{\varphi}^{2} \mathcal{J}\left(j_{\varphi}\right) \mathcal{D}\left(j_{\varphi}\right) \\
& +\frac{\lambda_{H}}{6}\left(85 y_{t}^{2}+25 y_{b}^{2}+85 y_{c}^{2}+75 y_{\tau}^{2}\right)-\frac{4}{3}\left(8 y_{t}^{4}-4 y_{b}^{4}+8 y_{c}^{4}+12 y_{\tau}^{4}\right) \text {, } \\
& B_{H, 02}=27 \lambda_{H}^{2}+2 \lambda_{\varphi H}^{2} \mathcal{J}\left(j_{\varphi}\right) \mathcal{D}\left(j_{\varphi}\right)+\left(\lambda_{\varphi H}^{\prime}\right)^{2} \mathcal{J}\left(j_{\varphi}\right) \mathcal{D}\left(j_{\varphi}\right)\left(\frac{\mathcal{J}\left(j_{\varphi}\right)}{6}-\frac{1}{8}\right) \\
& +\frac{15}{2} \lambda_{H}\left(3 y_{t}^{2}+3 y_{b}^{2}+3 y_{c}^{2}+y_{\tau}^{2}\right) \\
& B_{H, 00}=-\frac{39}{2} \lambda_{H}^{3}-\frac{\mathcal{D}\left(j_{\varphi}\right)}{4} \lambda_{\varphi H}^{3}-\frac{5}{8} \lambda_{\varphi H}^{2} \lambda_{H} \mathcal{D}\left(j_{\varphi}\right)-\frac{7}{96}\left(\lambda_{\varphi H}^{\prime}\right)^{2} \lambda_{H} \mathcal{J}\left(j_{\varphi}\right) \mathcal{D}\left(j_{\varphi}\right) \\
& -\frac{5}{48}\left(\lambda_{\varphi H}^{\prime}\right)^{2} \lambda_{\varphi H} \mathcal{J}\left(j_{\varphi}\right) \mathcal{D}\left(j_{\varphi}\right) \\
& -12 \lambda_{H}^{2}\left(3 y_{t}^{2}+3 y_{b}^{2}+3 y_{c}^{2}+y_{\tau}^{2}\right)+80 \lambda_{H} g_{s}^{2}\left(y_{t}^{2}+y_{b}^{2}+y_{c}^{2}\right) \\
& -\lambda_{H}\left(3 y_{t}^{4}+3 y_{b}^{4}+3 y_{c}^{4}+y_{\tau}^{4}+42 y_{t}^{2} y_{b}^{2}\right) \\
& +120\left(y_{t}^{6}+y_{b}^{6}+y_{c}^{6}\right)+40 y_{\tau}^{6}-24\left(y_{t}^{2} y_{b}^{4}+y_{t}^{4} y_{b}^{2}\right)-128 g_{s}^{2}\left(y_{t}^{4}+y_{b}^{4}+y_{c}^{4}\right) \text {; }
\end{aligned}
$$



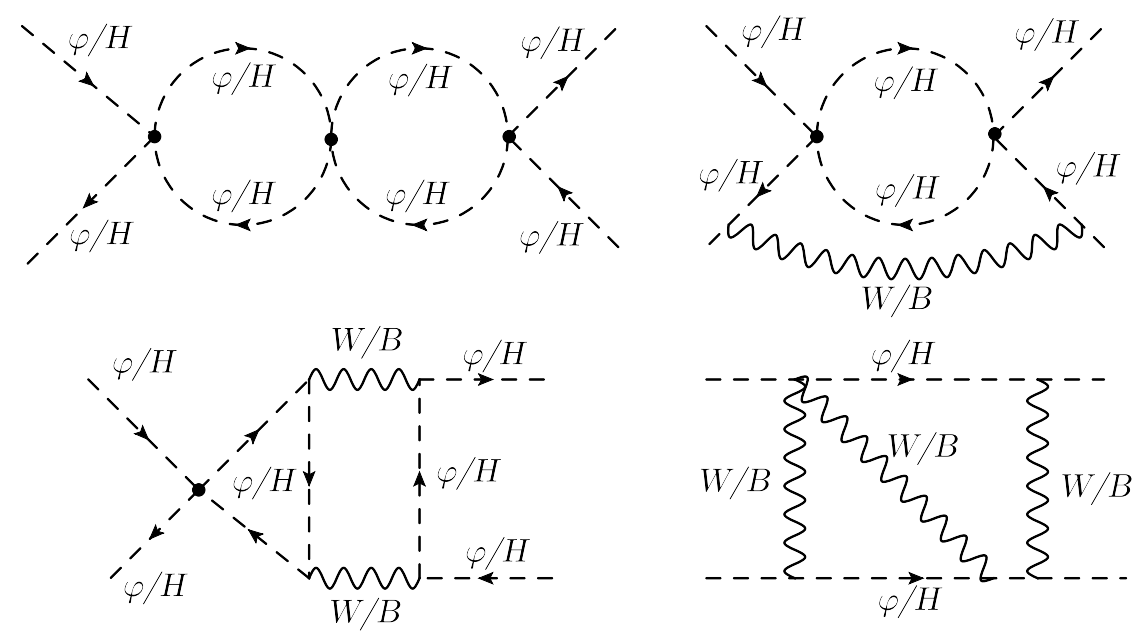

Figure 4. Sample Feynman diagrams which give contributions to quartic scalar couplings $\lambda_{H}$, $\lambda_{\varphi H}, \lambda_{\varphi H}^{\prime}$, and $\lambda_{\varphi}^{(J)}$ from $\varphi$. These diagrams divide into four classes: zero (top left), one (top right), two (bottom left), and three (bottom right) gauge boson insertions.

Our pure SM results agree with those in refs. [9, 11], apart from three terms which are consistent with the corrections made in ref. [13]. All other analytic results are presented here in closed form for the first time. ${ }^{2}$

For the two-loop calculation, we use the same strategy to express all SU(2) generators in terms of Sigma matrices and to simplify the expressions using the relations given in section 4. It is again possible to express all results in terms of the operators in the scalar potential (2.14), as required by gauge invariance.

At two-loops, all beta functions receive contributions from scalar fields. In the Yukawa beta functions, the only additional terms from scalar fields arise from the external Higgs field counterterm (figure 3b). The Feynman diagrams required to extract the quartic scalar coupling beta functions again split into different classes: those including zero, one, two, or three internal gauge bosons. In figure 4 , we give sample diagrams from each class which give contributions to the quartic scalar coupling beta functions.

\section{Group theory relations}

To express all results in terms of matrix elements of our basis operators, and to check the gauge-parameter independence and locality of our two-loop counterterms explicitly, we had to use a number of algebraic relations. These relations arise from the gauge invariance of the underlying theory as well as the properties of the Clebsch-Gordan coefficients, and are collected and proven below. For clarity, the summation convention is suspended in this section. All summations are indicated explicitly.

\footnotetext{
${ }^{2}$ As a cross check, we compared all our one- and two-loop beta functions with the results obtained using the code PyR@TE3 [26] for the two cases $j_{\varphi}=1, Y_{\varphi}=0$ and $j_{\varphi}=1, Y_{\varphi}=1$, and find complete agreement.
} 
To begin, we collect some orthogonality properties of the Sigma matrices that follow directly from the corresponding standard properties of the Clebsch-Gordan coefficients:

$$
\begin{aligned}
\sum_{k m} \Sigma_{k m}^{(J), M} \Sigma_{k m}^{\left(J^{\prime}\right), M^{\prime}} & =\delta^{J J^{\prime}} \delta^{M M^{\prime}}, \\
\sum_{M m} \Sigma_{k m}^{(J), M} \Sigma_{k^{\prime} m}^{(J), M} & =\frac{2 J+1}{2 j_{\varphi}+1} \delta_{k k^{\prime}}, \\
\sum_{J M} \Sigma_{k m}^{(J), M} \Sigma_{k^{\prime} m^{\prime}}^{(J), M} & =\delta_{k k^{\prime}} \delta_{m m^{\prime}}
\end{aligned}
$$

The exchange of $W$ gauge bosons introduces explicit $\mathrm{SU}(2)$ generators that need to be rewritten in terms of Sigma matrices. Since the Clebsch-Gordan coefficients describe a transformation between two complete sets of orthonormal state vectors, they are used to rewrite the product of two $\mathrm{SU}(2)$ generators:

$$
\sum_{a} \tilde{\tau}_{i r}^{a} \tilde{\tau}_{k l}^{a}=\sum_{J M} C(J) \Sigma_{i k}^{(J), M} \Sigma_{r l}^{(J), M}
$$

where the coefficient $C(J)$ is a function of $J$. The relation (4.4) is convenient since it can be applied recursively. Consider, for instance, the product of four generators:

$$
\sum_{a b} \sum_{l k} \tilde{\tau}_{i l}^{a} \tilde{\tau}_{l r}^{b} \tilde{\tau}_{m k}^{a} \tilde{\tau}_{k n}^{b}=\sum_{J M} \sum_{J^{\prime} M^{\prime}} \sum_{l k} C(J) C\left(J^{\prime}\right) \Sigma_{i m}^{(J), M} \Sigma_{l k}^{(J), M} \Sigma_{l k}^{\left(J^{\prime}\right), M^{\prime}} \Sigma_{r n}^{\left(J^{\prime}\right), M^{\prime}} .
$$

After applying the orthogonality relations, this becomes a linear combination of the basis operators,

$$
\sum_{a b} \sum_{l k} \tilde{\tau}_{i l}^{a} \tilde{\tau}_{l r}^{b} \tilde{\tau}_{m k}^{a} \tilde{\tau}_{k n}^{b}=\sum_{J M} C(J)^{2} \Sigma_{i m}^{(J), M} \Sigma_{r n}^{(J), M} .
$$

In fact, for a product of $2 n$ generators eq. (4.4) implies

$$
\sum_{a_{1} a_{2} \ldots a_{n}}\left(\tilde{\tau}^{a_{1}} \tilde{\tau}^{a_{2}} \ldots \tilde{\tau}^{a_{n}}\right)_{i r}\left(\tilde{\tau}^{a_{1}} \tilde{\tau}^{a_{2}} \ldots \tilde{\tau}^{a_{n}}\right)_{m q}=\sum_{J M} C(J)^{n} \Sigma_{i m}^{(J), M} \Sigma_{r q}^{(J), M}
$$

Diagrams with multiple scalar couplings likewise need to be expressed in terms of the basis operators. This is facilitated by the following "sum rule" for Sigma matrices:

$$
\sum_{M_{1} M_{2}} \sum_{m n} \Sigma_{i m}^{\left(J_{1}\right), M_{1}} \Sigma_{r n}^{\left(J_{1}\right), M_{1}} \Sigma_{k n}^{\left(J_{2}\right), M_{2}} \Sigma_{l m}^{\left(J_{2}\right), M_{2}}=\sum_{J_{3}, M_{3}} K\left(J_{1}, J_{2}, J_{3}\right) \Sigma_{i k}^{\left(J_{3}\right), M_{3}} \Sigma_{r l}^{\left(J_{3}\right), M_{3}} .
$$

In the following, we give explicit expressions for $C(J)$ and $K\left(J_{1}, J_{2}, J_{3}\right)$. We then derive further relations between these quantities that can be used to simplify the results of our calculation. Our general strategy is to express all results in terms of our operator basis and the group theory invariants $\mathcal{J}\left(j_{\varphi}\right) \equiv j_{\varphi}\left(j_{\varphi}+1\right)$, the eigenvalue of the $\mathrm{SU}(2)$ Casimir operator,

$$
\sum_{l} \tilde{\tau}_{i l}^{a} \tilde{\tau}_{l k}^{a}=\mathcal{J}\left(j_{\varphi}\right) \delta_{i k}
$$

and $\mathcal{D}\left(j_{\varphi}\right) \equiv 2 j_{\varphi}+1$, the dimension of the $\mathrm{SU}(2)$ multiplet representation with isospin $j_{\varphi}$. 
We begin by showing

$$
K\left(J_{1}, J_{2}, J_{3}\right)=\mathcal{D}\left(J_{1}\right) \mathcal{D}\left(J_{2}\right)\left\{\begin{array}{lll}
J_{1} & j_{\varphi} & j_{\varphi} \\
j_{\varphi} & J_{2} & j_{\varphi} \\
j_{\varphi} & j_{\varphi} & J_{3}
\end{array}\right\}
$$

in terms of the Wigner $9 j$ symbol [24]. Starting with eq. (4.8), we multiply both sides by $\Sigma_{r l}^{(J), M}$ and sum over $r, l$, to obtain

$$
\sum_{M_{1}, M_{2}} \sum_{m n r l} \Sigma_{i m}^{\left(J_{1}\right), M_{1}} \Sigma_{r n}^{\left(J_{1}\right), M_{1}} \Sigma_{k n}^{\left(J_{2}\right), M_{2}} \Sigma_{l m}^{\left(J_{2}\right), M_{2}} \Sigma_{r l}^{(J), M}=K\left(J_{1}, J_{2}, J\right) \Sigma_{i k}^{(J), M}
$$

The Sigma matrices can be written in terms of the Wigner $3 j$ symbols as [24]

$$
\Sigma_{m m^{\prime}}^{(J), M}=(-1)^{M} \sqrt{\mathcal{D}(J)}\left(\begin{array}{ccc}
j_{\varphi} & j_{\varphi} & J \\
m & m^{\prime} & -M
\end{array}\right)
$$

In this way, eq. (4.11) becomes

$$
\begin{aligned}
K\left(J_{1}, J_{2}, J\right)\left(\begin{array}{ccc}
j_{\varphi} & j_{\varphi} & J \\
i & k & -M
\end{array}\right)= & \sum_{M_{1}, M_{2}} \sum_{m n r l}(-1)^{-2 M_{1}-2 M_{2}} \mathcal{D}\left(J_{1}\right) \mathcal{D}\left(J_{2}\right) \\
& \times\left(\begin{array}{ccc}
j_{\varphi} & j_{\varphi} & J_{1} \\
i & m & -M_{1}
\end{array}\right)\left(\begin{array}{ccc}
j_{\varphi} & j_{\varphi} & J_{1} \\
r & n & -M_{1}
\end{array}\right) \\
& \times\left(\begin{array}{ccc}
j_{\varphi} & j_{\varphi} & J_{2} \\
k & n & -M_{2}
\end{array}\right)\left(\begin{array}{ccc}
j_{\varphi} & j_{\varphi} & J_{2} \\
l & m & -M_{2}
\end{array}\right)\left(\begin{array}{ccc}
j_{\varphi} & j_{\varphi} & J \\
r & l & -M
\end{array}\right)
\end{aligned}
$$

Since $M_{1}, M_{2} \in \mathbb{Z}$, the factor of -1 disappears. We can also freely change $-M_{1},-M_{2} \rightarrow$ $M_{1}, M_{2}$ since these indices are summed over. We also take $M \rightarrow-M$ on both sides. Now, we use the symmetry properties of the $3 j$ symbols

$$
\left(\begin{array}{ccc}
j_{1} & j_{2} & j_{3} \\
m_{1} & m_{2} & m_{3}
\end{array}\right)=\left(\begin{array}{ccc}
j_{2} & j_{3} & j_{1} \\
m_{2} & m_{3} & m_{1}
\end{array}\right)=(-1)^{j_{1}+j_{2}+j_{3}}\left(\begin{array}{ccc}
j_{1} & j_{3} & j_{2} \\
m_{1} & m_{3} & m_{2}
\end{array}\right)
$$

to rewrite

$$
\begin{aligned}
K\left(J_{1}, J_{2}, J\right)\left(\begin{array}{ccc}
j_{\varphi} & j_{\varphi} & J \\
i & k & M
\end{array}\right)= & \sum_{M_{1}, M_{2}} \sum_{m n r l}(-1)^{2 J_{1}+2 J_{2}+8 j_{\varphi}} \mathcal{D}\left(J_{1}\right) \mathcal{D}\left(J_{2}\right) \\
& \times\left(\begin{array}{ccc}
J_{1} & j_{\varphi} & j_{\varphi} \\
M_{1} & n & r
\end{array}\right)\left(\begin{array}{ccc}
j_{\varphi} & J_{2} & j_{\varphi} \\
m & M_{2} & l
\end{array}\right) \\
& \times\left(\begin{array}{ccc}
J_{1} & j_{\varphi} & j_{\varphi} \\
M_{1} & m & i
\end{array}\right)\left(\begin{array}{ccc}
j_{\varphi} & J_{2} & j_{\varphi} \\
n & M_{2} & k
\end{array}\right)\left(\begin{array}{ccc}
j_{\varphi} & j_{\varphi} & J \\
r & l & M
\end{array}\right) .
\end{aligned}
$$


The Wigner $9 j$ symbols are written in terms of the $3 j$ symbols as [24]

$$
\begin{aligned}
& \left(\begin{array}{ccc}
J_{13} & J_{24} & J \\
M_{13} & M_{24} & M
\end{array}\right)\left\{\begin{array}{ccc}
j_{1} & j_{2} & J_{12} \\
j_{3} & j_{4} & J_{34} \\
J_{13} & J_{24} & J
\end{array}\right\} \\
= & \sum_{\substack{m_{1} m_{2} m_{3} m_{4} \\
M_{12} M_{34}}}\left(\begin{array}{ccc}
j_{1} & j_{2} & J_{12} \\
m_{1} & m_{2} & M_{12}
\end{array}\right)\left(\begin{array}{ccc}
j_{3} & j_{4} & J_{34} \\
m_{3} & m_{4} & M_{34}
\end{array}\right) \\
& \times\left(\begin{array}{ccc}
j_{1} & j_{3} & J_{13} \\
m_{1} & m_{3} & M_{13}
\end{array}\right)\left(\begin{array}{ccc}
j_{2} & j_{4} & J_{24} \\
m_{2} & m_{4} & M_{24}
\end{array}\right)\left(\begin{array}{ccc}
J_{12} & J_{34} & J \\
M_{12} & M_{34} & M
\end{array}\right) .
\end{aligned}
$$

Comparison of the last two equation yields eq. (4.10). Note that, as expected, $K\left(J_{1}, J_{2}, J_{3}\right)$ is symmetric in its first two indices.

Next, we show

$$
C(J)=\frac{1}{2} \mathcal{J}(J)-\mathcal{J}\left(j_{\varphi}\right)
$$

First, we contract eq. (4.4) with two Sigma matrices and use the orthogonality relation (4.1) to arrive at

$$
\mathcal{D}(J) C(J)=\sum_{a} \sum_{M} \sum_{i r k l} \tilde{\tau}_{i r}^{a} \tilde{\tau}_{k l}^{a} \Sigma_{i k}^{(J), M} \Sigma_{r l}^{(J), M} .
$$

We find $C(J)$ by writing eq. (4.18) explicitly in terms of Clebsch-Gordan coefficients as

$$
\begin{aligned}
\mathcal{D}(J) C(J)=\sum_{a} \sum_{M} \sum_{m n m^{\prime} n^{\prime}} & C_{j_{\varphi} j_{\varphi}}(J M ; m n) C_{j_{\varphi} j_{\varphi}}\left(J M ; m^{\prime} n^{\prime}\right) \\
& \times\left\langle j_{\varphi} m\left|\tilde{\tau}^{\left(j_{\varphi}\right), a}\right| j_{\varphi} m^{\prime}\right\rangle\left\langle j_{\varphi} n\left|\tilde{\tau}^{\left(j_{\varphi}\right), a}\right| j_{\varphi} n^{\prime}\right\rangle .
\end{aligned}
$$

Noting that, by definition, $\tilde{\tau}^{\left(j_{\varphi}\right), a}$ are the $\operatorname{spin}-j_{\varphi}$ generators, we introduce the notation $\tilde{\tau}_{m n}^{a} \equiv\left\langle j_{\varphi} m\left|\tilde{\tau}^{\left(j_{\varphi}\right), a}\right| j_{\varphi} n\right\rangle$, i.e. we label the generators with $j_{\varphi}$ as well as $a=1,2,3$, and the states by $j_{\varphi}$ and their "magnetic" quantum numbers $m, n, m,{ }^{\prime} n^{\prime}=-j_{\varphi}, \ldots, j_{\varphi}$. Using the symmetry relation of the Clebsch-Gordan coefficients

$$
C_{j j}(J M ; m n)=(-1)^{j+m} \sqrt{\frac{\mathcal{D}(J)}{\mathcal{D}(j)}} C_{j J}(j,-n ; m,-M),
$$

eq. (4.19) becomes

$$
\begin{aligned}
\mathcal{D}(J) C(J)=\frac{\mathcal{D}(J)}{\mathcal{D}\left(j_{\varphi}\right)} \sum_{a} \sum_{M} \sum_{m m^{\prime} n n^{\prime}} & (-1)^{2 j_{\varphi}-n-n^{\prime}} \\
& \times C_{j_{\varphi} J}\left(j_{\varphi},-n ; m M\right) C_{j_{\varphi} J}\left(j_{\varphi},-n^{\prime} ; m^{\prime} M\right) \\
& \times\left\langle j_{\varphi} m\left|\tilde{\tau}^{\left(j_{\varphi}\right), a}\right| j_{\varphi} m^{\prime}\right\rangle\left\langle j_{\varphi} n\left|\tilde{\tau}^{\left(j_{\varphi}\right), a}\right| j_{\varphi} n^{\prime}\right\rangle
\end{aligned}
$$

where we use $m=M-n$ and $m^{\prime}=M-n^{\prime}$, as well as the fact that $M$ is always integer, to rewrite the phase factor. We also take $-M \rightarrow M$ using the symmetry of the sum over 
$M$. Now, we artificially regard each spin- $j_{\varphi}$ state as belonging to the spin- $j_{\varphi}$ subspace of the Clebsch-Gordan decomposition of the tensor product of a spin- $j_{\varphi}$ and a spin- $J$ state. For instance, $\left\langle j_{\varphi} m\right|=\sum_{n N} C_{j_{\varphi} J}\left(j_{\varphi} m ; n N\right)\left\langle j_{\varphi} n ; J N\right|$ and analogous relations lead to

$$
\begin{aligned}
& \left\langle j_{\varphi} m\left|\tilde{\tau}^{\left(j_{\varphi}\right), a}\right| j_{\varphi} m^{\prime}\right\rangle \\
& =\sum_{n N} \sum_{n^{\prime} N^{\prime}} C_{j_{\varphi} J}\left(j_{\varphi} m ; n N\right) C_{j_{\varphi} J}\left(j_{\varphi} m^{\prime} ; n^{\prime} N^{\prime}\right)\left\langle j_{\varphi} n, J N\left|\left(\tilde{\tau}^{\left(j_{\varphi}\right), a} \otimes \mathbb{1}_{J}+\mathbb{1}_{j_{\varphi}} \otimes \tilde{\tau}^{(J), a}\right)\right| j_{\varphi} n^{\prime}, J N^{\prime}\right\rangle .
\end{aligned}
$$

Hence, we find the explicit tensor decomposition for the generators

$$
\begin{aligned}
\tilde{\tau}_{m n}^{\left(j_{\varphi}\right), a}= & \sum_{\tilde{M}} \sum_{\tilde{m} \tilde{n}} C_{j_{\varphi} J}\left(j_{\varphi} m ; \tilde{m} \tilde{M}\right) C_{j_{\varphi} J}\left(j_{\varphi} n ; \tilde{n} \tilde{M}\right) \tilde{\tau}_{\tilde{m} \tilde{n}}^{\left(j_{\varphi}\right), a} \\
& +\sum_{\tilde{M} \tilde{N}} \sum_{\tilde{m}} C_{j_{\varphi} J}\left(j_{\varphi} m ; \tilde{m} \tilde{M}\right) C_{j_{\varphi} J}\left(j_{\varphi} n ; \tilde{m} \tilde{N}\right) \tilde{\tau}_{\tilde{M} \tilde{N}}^{(J), a} .
\end{aligned}
$$

Inserting this identity into eq. (4.21) gives

$$
\begin{aligned}
\mathcal{D} & (J) C(J) \\
= & \frac{\mathcal{D}(J)}{\mathcal{D}\left(j_{\varphi}\right)} \sum_{a} \sum_{n n^{\prime}}(-1)^{2 j_{\varphi}-n-n^{\prime}} \tilde{\tau}_{-n,-n^{\prime}}^{\left(j_{\varphi}\right), a} \tilde{\tau}_{n n^{\prime}}^{\left(j_{\varphi}\right), a} \\
& -\frac{\mathcal{D}(J)}{\mathcal{D}\left(j_{\varphi}\right)} \sum_{a} \sum_{\tilde{m}} \sum_{M M^{\prime} n n^{\prime}}(-1)^{2 j_{\varphi}-n-n^{\prime}} C_{j_{\varphi} J}\left(j_{\varphi},-n ; \tilde{m} M\right) C_{j_{\varphi} J}\left(j_{\varphi},-n^{\prime} ; \tilde{m} M^{\prime}\right) \tilde{\tau}_{M M^{\prime}}^{(J), a} \tilde{\tau}_{n n^{\prime}}^{\left(j_{\varphi}\right), a} \\
= & \frac{\mathcal{D}(J)}{\mathcal{D}\left(j_{\varphi}\right)} \sum_{a} \sum_{n n^{\prime}}(-1)^{2 j_{\varphi}+n+n^{\prime}} \tilde{\tau}_{-n,-n^{\prime}}^{\left(j_{\varphi}\right), a} \tilde{\tau}_{n n^{\prime}}^{\left(j_{\varphi}\right), a} \\
& -\sum_{a} \sum_{\tilde{m}} \sum_{M M^{\prime} n n^{\prime}}(-1)^{2 J+M+M^{\prime} C_{j_{\varphi} j_{\varphi}}(J,-M ; \tilde{m} n) C_{j_{\varphi} j_{\varphi}}\left(J,-M^{\prime} ; \tilde{m} n^{\prime}\right) \tilde{\tau}_{M M}^{(J), a} \tilde{\tau}_{n n^{\prime}}^{\left(j_{\varphi}\right), a}}
\end{aligned}
$$

where we again use eq. (4.20) and rewrite the phase in the second term noting that $\tilde{m}=-n-M=-n^{\prime}-M^{\prime}$ and $(-1)^{2 J}=1$. We then use an identity analogous to (4.23), namely,

$$
\begin{aligned}
\tilde{\tau}_{M N}^{(J), a}= & \sum_{\tilde{m}} \sum_{m^{\prime} n^{\prime}} C_{j_{\varphi} j_{\varphi}}\left(J M ; m^{\prime} \tilde{m}\right) C_{j_{\varphi} j_{\varphi}}\left(J N ; n^{\prime} \tilde{m}\right) \tilde{\tau}_{m^{\prime} n^{\prime}}^{\left(j_{\varphi}\right), a} \\
& +\sum_{\tilde{m}} \sum_{m^{\prime} n^{\prime}} C_{j_{\varphi} j_{\varphi}}\left(J M ; \tilde{m} m^{\prime}\right) C_{j_{\varphi} j_{\varphi}}\left(J N ; \tilde{m} n^{\prime}\right) \tilde{\tau}_{m^{\prime} n^{\prime}}^{\left(j_{\varphi}\right), a},
\end{aligned}
$$

to obtain

$$
\sum_{\tilde{m}} \sum_{m^{\prime} n^{\prime}} C_{j_{\varphi} j_{\varphi}}\left(J M ; \tilde{m} m^{\prime}\right) C_{j_{\varphi} j_{\varphi}}\left(J N ; \tilde{m} n^{\prime}\right) \tilde{\tau}_{m^{\prime} n^{\prime}}^{\left(j_{\varphi}\right), a}=\frac{1}{2} \tilde{\tau}_{M N}^{(J), a}
$$

and so

$$
\begin{aligned}
\mathcal{D}(J) C(J)= & \frac{\mathcal{D}(J)}{\mathcal{D}\left(j_{\varphi}\right)} \sum_{a} \sum_{n n^{\prime}}(-1)^{2 j_{\varphi}+n+n^{\prime}} \tilde{\tau}_{-n,-n^{\prime}}^{\left(j_{\varphi}\right), a} \tilde{\tau}_{n n^{\prime}}^{\left(j_{\varphi}\right), a} \\
& -\frac{1}{2} \sum_{a} \sum_{M M^{\prime}}(-1)^{2 J+M+M^{\prime}} \tilde{\tau}_{-M,-M^{\prime}}^{(J), a} \tilde{\tau}_{M M^{\prime}}^{(J), a}
\end{aligned}
$$


A straightforward calculation using the explicit expressions for the spin- $j_{\varphi}$ generators, eq. (2.4), gives

$$
\begin{aligned}
(-1)^{2 j_{\varphi}+n+n^{\prime}} \tilde{\tau}_{-n,-n^{\prime}}^{\left(j_{\varphi}\right), 1} \tilde{\tau}_{n n^{\prime}}^{\left(j_{\varphi}\right), 1} & =(-1)^{2 j_{\varphi}+n+n^{\prime}} \tilde{\tau}_{-n,-n^{\prime}}^{\left(j_{\varphi}\right), 2} \tilde{\tau}_{n n^{\prime}}^{\left(j_{\varphi}\right), 2} \\
& =-\frac{1}{4}\left[\delta_{n, n^{\prime}+1}^{2}\left(j_{\varphi}+n^{\prime}+1\right)\left(j_{\varphi}-n^{\prime}\right)+\delta_{n, n^{\prime}-1}^{2}\left(j_{\varphi}-n^{\prime}+1\right)\left(j_{\varphi}+n^{\prime}\right)\right], \\
(-1)^{2 j_{\varphi}+n+n^{\prime}} \tilde{\tau}_{-n,-n^{\prime}}^{\left(j_{\varphi}\right), 3} \tilde{\tau}_{n n^{\prime}}^{\left(j_{\varphi}\right), 3} & =-n^{2} \delta_{n n^{\prime}}^{2} .
\end{aligned}
$$

Comparing these to the analogous expressions

$$
\begin{aligned}
\tilde{\tau}_{n^{\prime} n}^{\left(j_{\varphi}\right), 1} \tilde{\tau}_{n n^{\prime}}^{\left(j_{\varphi}\right), 1} & =\tilde{\tau}_{n^{\prime} n}^{\left(j_{\varphi}\right), 2} \tilde{\tau}_{n n^{\prime}}^{\left(j_{\varphi}\right), 2} \\
& =\frac{1}{4}\left[\delta_{n, n^{\prime}+1}^{2}\left(j_{\varphi}+n^{\prime}+1\right)\left(j_{\varphi}-n^{\prime}\right)+\delta_{n, n^{\prime}-1}^{2}\left(j_{\varphi}-n^{\prime}+1\right)\left(j_{\varphi}+n^{\prime}\right)\right] \\
\tilde{\tau}_{n^{\prime} n}^{\left(j_{\varphi}\right), 3} \tilde{\tau}_{n n^{\prime}}^{\left(j_{\varphi}\right), 3} & =n^{2} \delta_{n n^{\prime}}^{2}
\end{aligned}
$$

we see that

$$
\sum_{a} \sum_{n n^{\prime}}(-1)^{2 j_{\varphi}+k+l} \tilde{\tau}_{-n,-n^{\prime}}^{\left(j_{\varphi}\right), a} \tilde{\tau}_{n n^{\prime}}^{\left(j_{\varphi}\right), a}=-\sum_{a} \sum_{n n^{\prime}} \tilde{\tau}_{n^{\prime} n}^{\left(j_{\varphi}\right), a} \tilde{\tau}_{n n^{\prime}}^{\left(j_{\varphi}\right), a}=-j_{\varphi}\left(j_{\varphi}+1\right)\left(2 j_{\varphi}+1\right) .
$$

This yields the desired result (4.17).

In the following, we collect several sum rules involving the coefficient $C(J)$. The first is

$$
\sum_{J} C(J) \mathcal{D}(J)=0
$$

It is derived by summing eq. (4.18) over $J$ and using the orthogonality relations, to obtain

$$
\sum_{J} C(J) \mathcal{D}(J)=\sum_{a} \sum_{i r k l} \tilde{\tau}_{i r}^{a} \tilde{\tau}_{k l}^{a} \delta_{i r} \delta_{k l}=0
$$

where we use the fact that the generators are traceless. In practice, the interchange of indices in the Sigma matrices gives rise to additional phase factors, hence we also need the relation

$$
\sum_{J}(-1)^{J-2 j_{\varphi}} C(J) \mathcal{D}(J)=\mathcal{D}\left(j_{\varphi}\right) \mathcal{J}\left(j_{\varphi}\right)
$$

To prove this relation, we again sum eq. (4.18) over $J$, now taking into account the symmetry properties of the Clebsch-Gordan coefficients

$$
\sum_{J}(-1)^{J-2 j_{\varphi}} C(J) \mathcal{D}(J)=\sum_{a} \sum_{i r k l} \tilde{\tau}_{i r}^{a} \tilde{\tau}_{k l}^{a} \delta_{i l} \delta_{k r}=\sum_{a} \sum_{i r} \tilde{\tau}_{i r}^{a} \tilde{\tau}_{r i}^{a}=\mathcal{D}\left(j_{\varphi}\right) \mathcal{J}\left(j_{\varphi}\right) .
$$

We note in passing that this relation can be used to calculate the "restricted" sum over $J$, as

$$
\sum_{J}^{\prime} C(J) \mathcal{D}(J) \equiv \sum_{J} \frac{1+(-1)^{J-2 j_{\varphi}}}{2} C(J) \mathcal{D}(J)=\frac{1}{2} \mathcal{D}\left(j_{\varphi}\right) \mathcal{J}\left(j_{\varphi}\right) .
$$


A similar sum rule, quadratic in $C(J)$, reads

$$
\sum_{J} \mathcal{D}(J) C(J)^{2}=\frac{\mathcal{J}\left(j_{\varphi}\right)^{2} \mathcal{D}\left(j_{\varphi}\right)^{2}}{3}
$$

To see this, we rewrite the left-hand side as

$$
\sum_{J} \mathcal{D}(J) C(J)^{2}=\sum_{a b} \sum_{J M} \sum_{i r k l m n} \tilde{\tau}_{i m}^{a} \tilde{\tau}_{m r}^{b} \tilde{\tau}_{k n}^{a} \tilde{\tau}_{n l}^{b} \Sigma_{i k}^{(J), M} \Sigma_{r l}^{(J), M}=\sum_{a b} \sum_{i m k n} \tilde{\tau}_{i m}^{a} \tilde{\tau}_{m i}^{b} \tilde{\tau}_{k n}^{a} \tilde{\tau}_{n k}^{b} .
$$

Now, we use the $\mathrm{SU}(2)$ relation

$$
\sum_{i m} \tilde{\tau}_{i m}^{a} \tilde{\tau}_{m i}^{b}=\frac{\mathcal{J}\left(j_{\varphi}\right) \mathcal{D}\left(j_{\varphi}\right)}{3} \delta^{a b}
$$

to find eq. (4.36). The analogous sum rule with phase factor reads

$$
\sum_{J}(-1)^{J-2 j_{\varphi}} \mathcal{D}(J) C(J)^{2}=\mathcal{J}\left(j_{\varphi}\right) \mathcal{D}\left(j_{\varphi}\right)\left(\mathcal{J}\left(j_{\varphi}\right)-1\right)
$$

The proof proceeds similar to the above, except we must use the (anti-)symmetry of the Clebsch-Gordan coefficients:

$$
\sum_{J}(-1)^{J-2 j_{\varphi}} \mathcal{D}(J) C(J)^{2}=\sum_{a b} \sum_{i m k n} \tilde{\tau}_{i m}^{a} \tilde{\tau}_{m k}^{b} \tilde{\tau}_{k n}^{a} \tilde{\tau}_{n i}^{b} .
$$

We then use the $\mathrm{SU}(2)$ algebra to re-write

$$
\begin{aligned}
\sum_{a b} \sum_{i m k n} \tilde{\tau}_{i m}^{a} \tilde{\tau}_{m k}^{b} \tilde{\tau}_{k n}^{a} \tilde{\tau}_{n i}^{b} & =\sum_{a b} \sum_{i m k n} \tilde{\tau}_{i m}^{a} \tilde{\tau}_{m k}^{a} \tilde{\tau}_{k n}^{b} \tilde{\tau}_{n i}^{b}+\sum_{a b c} \sum_{i m n} i \epsilon^{b a c} \tilde{\tau}_{i m}^{a} \tilde{\tau}_{m n}^{c} \tilde{\tau}_{n i}^{b} \\
& =\mathcal{J}\left(j_{\varphi}\right)^{2} \mathcal{D}\left(j_{\varphi}\right)-\mathcal{J}\left(j_{\varphi}\right) \mathcal{D}\left(j_{\varphi}\right),
\end{aligned}
$$

which gives the relation (4.39). Again, we use this to calculate the restricted sum over $J$, as

$$
\begin{aligned}
\sum_{J}^{\prime} \mathcal{D}(J) C(J)^{2} & =\sum_{J} \frac{1+(-1)^{J-2 j_{\varphi}}}{2} \mathcal{D}(J) C(J)^{2} \\
& =\frac{1}{6} \mathcal{J}\left(j_{\varphi}\right) \mathcal{D}\left(j_{\varphi}\right)\left(\mathcal{J}\left(j_{\varphi}\right) \mathcal{D}\left(j_{\varphi}\right)+3 \mathcal{J}\left(j_{\varphi}\right)-3\right)
\end{aligned}
$$

Another pair of rules cubic in $C(J)$ is necessary to reduce the algebra in diagrams involving the SM Higgs. The first is given by

$$
\sum_{J} \mathcal{D}(J) C(J)^{3}=-\frac{\mathcal{J}\left(j_{\varphi}\right)^{2} \mathcal{D}\left(j_{\varphi}\right)^{2}}{6}
$$

Rewriting the left-hand side and using the appopriate orthogonality relations for the Clebsch-Gordan coefficients gives

$$
\sum_{J} \mathcal{D}(J) C(J)^{3}=\sum_{a b c} \operatorname{Tr}\left[\tilde{\tau}^{a} \tilde{\tau}^{b} \tilde{\tau}^{c}\right] \operatorname{Tr}\left[\tilde{\tau}^{a} \tilde{\tau}^{b} \tilde{\tau}^{c}\right] .
$$


The trace of three generators is expressed as

$$
\operatorname{Tr}\left[\tilde{\tau}^{a} \tilde{\tau}^{b} \tilde{\tau}^{c}\right]=\frac{1}{2}\left(\operatorname{Tr}\left[\left\{\tilde{\tau}^{a}, \tilde{\tau}^{b}\right\} \tilde{\tau}^{c}\right]+\operatorname{Tr}\left[\left[\tilde{\tau}^{a}, \tilde{\tau}^{b}\right] \tilde{\tau}^{c}\right]\right)=\frac{1}{2} \operatorname{Tr}\left[\left[\tilde{\tau}^{a}, \tilde{\tau}^{b}\right] \tilde{\tau}^{c}\right],
$$

where we make use of the definition of the totally symmetric tensor,

$$
d^{a b c} \propto \operatorname{Tr}\left[\left\{\tilde{\tau}^{a}, \tilde{\tau}^{b}\right\} \tilde{\tau}^{c}\right],
$$

which vanishes for $\mathrm{SU}(2)$. Next, we simply use the group algebra to find

$$
\operatorname{Tr}\left[\tilde{\tau}^{a} \tilde{\tau}^{b} \tilde{\tau}^{c}\right]=\frac{i \mathcal{J}\left(j_{\varphi}\right) \mathcal{D}\left(j_{\varphi}\right)}{6} \epsilon^{a b c} .
$$

Squaring this expression and using

$$
\sum_{a b c} \epsilon^{a b c} \epsilon^{a b c}=6
$$

gives the result in eq. (4.43). The second relation cubic in $C(J)$ is

$$
\sum_{J}(-1)^{J-2 j_{\varphi}} \mathcal{D}(J) C(J)^{3}=\mathcal{J}\left(j_{\varphi}\right) \mathcal{D}\left(j_{\varphi}\right)\left(\mathcal{J}\left(j_{\varphi}\right)-1\right)\left(\mathcal{J}\left(j_{\varphi}\right)-2\right)
$$

As before, we re-write the left-hand side of this expression and use orthogonality relations

$$
\sum_{J}(-1)^{J-2 j_{\varphi}} \mathcal{D}(J) C(J)^{3}=\sum_{a b c} \operatorname{Tr}\left[\tilde{\tau}^{a} \tilde{\tau}^{b} \tilde{\tau}^{c} \tilde{\tau}^{a} \tilde{\tau}^{b} \tilde{\tau}^{c}\right] .
$$

The product in the trace simplifies using the $\mathrm{SU}(2)$ algebra

$$
\sum_{a} \tilde{\tau}^{a} \tilde{\tau}^{b} \tilde{\tau}^{c} \tilde{\tau}^{a}=\sum_{a}\left(\tilde{\tau}^{a} \tilde{\tau}^{b} \tilde{\tau}^{a} \tilde{\tau}^{c}+i \epsilon^{c a d} \tilde{\tau}^{a} \tilde{\tau}^{b} \tilde{\tau}^{d}\right)
$$

Then, using

$$
\sum_{a} \tilde{\tau}^{a} \tilde{\tau}^{b} \tilde{\tau}^{a}=\left(\mathcal{J}\left(j_{\varphi}\right)-1\right) \tilde{\tau}^{b}
$$

the trace is reduced to

$$
\sum_{a b c} \operatorname{Tr}\left[\tilde{\tau}^{a} \tilde{\tau}^{b} \tilde{\tau}^{c} \tilde{\tau}^{a} \tilde{\tau}^{b} \tilde{\tau}^{c}\right]=\operatorname{Tr}\left[\mathcal{J}\left(j_{\varphi}\right)\left(\mathcal{J}\left(j_{\varphi}\right)-1\right)^{2} \mathbb{1}-\mathcal{J}\left(j_{\varphi}\right)\left(\mathcal{J}\left(j_{\varphi}\right)-1\right) \mathbb{1}\right]
$$

which, when the trace is performed over the identity, gives eq. (4.49).

In order to derive the necessary algebraic relations involving the factor $K$, we first prove the following useful relation:

$$
\sum_{M} \sum_{k l} \Sigma_{i k}^{(J), M} \Sigma_{r l}^{(J), M} \tilde{\tau}_{l k}^{a}=\frac{\mathcal{D}(J) C(J)}{\mathcal{J}\left(j_{\varphi}\right) \mathcal{D}\left(j_{\varphi}\right)} \tilde{\tau}_{i r}^{a}
$$

To derive this, we note that the only object in our basis with a single adjoint representation index and two isospin- $j_{\varphi}$ representation indices is the generator $\tilde{\tau}_{i r}^{a}$ (any other objects with only these free indices can be reduced to this generator). Therefore, we make the ansatz

$$
\sum_{M} \sum_{k l} \Sigma_{i k}^{(J), M} \Sigma_{r l}^{(J), M} \tilde{\tau}_{l k}^{a}=G(J) \tilde{\tau}_{i r}^{a} .
$$


Multiplying both sides by $\tilde{\tau}_{r i}^{a}$, summing over $a, i, r$, and using eq. (4.18) gives the relation (4.54). Using this result, we now prove

$$
\sum_{J_{1}} C\left(J_{1}\right) K\left(J_{1}, J_{2}, J_{3}\right)=\frac{\mathcal{D}\left(J_{2}\right) C\left(J_{2}\right) C\left(J_{3}\right)}{\mathcal{J}\left(j_{\varphi}\right) \mathcal{D}\left(j_{\varphi}\right)}
$$

To this end, we consider the product

$$
\begin{aligned}
\sum_{a, m n} \sum_{M} \tilde{\tau}_{i r}^{a} \tilde{\tau}_{m n}^{a} \Sigma_{k n}^{(J), M} \Sigma_{l m}^{(J), M} & =\frac{\mathcal{D}(J) C(J)}{\mathcal{J}\left(j_{\varphi}\right) \mathcal{D}\left(j_{\varphi}\right)} \sum_{a} \tilde{\tau}_{i r}^{a} \tilde{\tau}_{k l}^{a} \\
& =\frac{\mathcal{D}(J) C(J)}{\mathcal{J}\left(j_{\varphi}\right) \mathcal{D}\left(j_{\varphi}\right)} \sum_{J_{1} M_{1}} C\left(J_{1}\right) \Sigma_{i k}^{\left(J_{1}\right), M_{1}} \Sigma_{r l}^{\left(J_{1}\right), M_{1}}
\end{aligned}
$$

making use of eqs. (4.54) and (4.4). However, this is alternatively written as

$$
\begin{aligned}
\sum_{a, m n} \sum_{M} \tilde{\tau}_{i r}^{a} \tilde{\tau}_{m n}^{a} \Sigma_{k n}^{(J), M} \Sigma_{l m}^{(J), M} & =\sum_{m n} \sum_{J_{1}, M_{1}, M} C\left(J_{1}\right) \Sigma_{k n}^{(J), M} \Sigma_{l m}^{(J), M} \Sigma_{i m}^{\left(J_{1}\right), M_{1}} \Sigma_{r n}^{\left(J_{1}\right), M_{1}} \\
& =\sum_{J_{1}, J_{2}, M_{2}} C\left(J_{1}\right) K\left(J_{1}, J, J_{2}\right) \Sigma_{i k}^{\left(J_{2}\right), M_{2}} \Sigma_{r l}^{\left(J_{2}\right), M_{2}} .
\end{aligned}
$$

Equating these expressions and using the orthogonality relations, the result (4.56) follows.

A further important relation incorporates the condition of gauge invariance:

$$
\sum_{J_{1}}(-1)^{J_{1}-2 j_{\varphi}}\left(1+(-1)^{J-2 j_{\varphi}}(-1)^{J_{2}-2 j_{\varphi}}\right) C\left(J_{1}\right) K\left(J, J_{1}, J_{2}\right)=(\mathcal{J}(j)+C(J)) \delta^{J, J_{2}}
$$

All interaction vertices must be gauge-invariant. A scalar $\mathrm{SU}(2)$ spin- $j_{\varphi}$ field multiplet transforms as $\varphi_{i} \rightarrow \varphi_{i}^{\prime}=\varphi_{i}+\delta \varphi_{i}$ under an infinitesimal gauge transformation, where

$$
\delta \varphi_{i}=\sum_{a, k} i \epsilon^{a} \tilde{\tau}_{i k}^{a} \varphi_{k}-\frac{1}{2} \sum_{a b, l k} \epsilon^{a} \epsilon^{b} \tilde{\tau}_{i l}^{a} \tilde{\tau}_{l k}^{b} \varphi_{k}+\mathcal{O}\left(\epsilon^{3}\right)
$$

while

$$
\delta \varphi_{i}^{*}=-\sum_{a, k} i \epsilon^{a} \tilde{\tau}_{k i}^{a} \varphi_{k}^{*}-\frac{1}{2} \sum_{a b, l k} \epsilon^{a} \epsilon^{b} \tilde{\tau}_{k l}^{a} \tilde{\tau}_{l i}^{b} \varphi_{k}^{*}+\mathcal{O}\left(\epsilon^{3}\right) .
$$

Hence, we have the relation

$$
\begin{aligned}
0=\sum_{m M}\left(\tilde{\tau}_{m i}^{a} \Sigma_{m k}^{(J), M} \Sigma_{r l}^{(J), M}+\tilde{\tau}_{m k}^{a} \Sigma_{i m}^{(J), M} \Sigma_{r l}^{(J), M}\right. \\
\left.\quad-\tilde{\tau}_{r m}^{a} \Sigma_{i k}^{(J), M} \Sigma_{m l}^{(J), M}-\tilde{\tau}_{l m}^{a} \Sigma_{i k}^{(J), M} \Sigma_{r m}^{(J), M}\right),
\end{aligned}
$$

which leads, upon contraction with a $\mathrm{SU}(2)$ generator, to

$$
\begin{aligned}
\mathcal{J}\left(j_{\varphi}\right) & \sum_{M} \Sigma_{i k}^{(J), M} \Sigma_{r n}^{(J), M} \\
& =\sum_{l m, a, M}\left(\tilde{\tau}_{n l}^{a} \tilde{\tau}_{m i}^{a} \Sigma_{m k}^{(J), M} \Sigma_{r l}^{(J), M}+\tilde{\tau}_{n l}^{a} \tilde{\tau}_{m k}^{a} \Sigma_{i m}^{(J), M} \Sigma_{r l}^{(J), M}-\tilde{\tau}_{n l}^{a} \tilde{\tau}_{r m}^{a} \Sigma_{i k}^{(J), M} \Sigma_{m l}^{(J), M}\right) .
\end{aligned}
$$


We use eqs. (4.4) and (4.8) to rewrite this condition as

$$
\begin{aligned}
& \mathcal{J}\left(j_{\varphi}\right) \sum_{M} \Sigma_{i k}^{(J), M} \Sigma_{r n}^{(J), M}=\sum_{J_{1}} \sum_{M, M_{1}} \sum_{m l} C\left(J_{1}\right)\left(\Sigma_{m k}^{(J), M} \Sigma_{r l}^{(J), M} \Sigma_{n m}^{\left(J_{1}\right), M_{1}} \Sigma_{l i}^{\left(J_{1}\right), M_{1}}\right. \\
& +\Sigma_{i m}^{(J), M} \Sigma_{r l}^{(J), M} \Sigma_{n m}^{\left(J_{1}\right), M_{1}} \Sigma_{l k}^{\left(J_{1}\right), M_{1}} \\
& \left.\quad-\Sigma_{i k}^{(J), M} \Sigma_{m l}^{(J), M} \Sigma_{n r}^{\left(J_{1}\right), M_{1}} \Sigma_{l m}^{\left(J_{1}\right), M_{1}}\right) \\
& =\sum_{J_{1}, J_{2}, M_{2}}(-1)^{J_{1}-2 j_{\varphi}} C\left(J_{1}\right) K\left(J, J_{1}, J_{2}\right)\left[(-1)^{J-2 j \varphi} \Sigma_{i k}^{\left(J_{2}\right), M_{2}} \Sigma_{n r}^{\left(J_{2}\right), M_{2}}+\Sigma_{i k}^{\left(J_{2}\right), M_{2}} \Sigma_{r n}^{\left(J_{2}\right), M_{2}}\right] \\
& \quad-C(J) \sum_{M} \Sigma_{i k}^{(J), M} \Sigma_{r n}^{(J), M}
\end{aligned}
$$

Multiplying both sides by $\sum_{M_{3}} \Sigma_{i k}^{\left(J_{3}\right), M_{3}} \Sigma_{r n}^{\left(J_{3}\right), M_{3}}$ and summing over $i, k, r, n$ yields eq. (4.59). We now derive a few relations involving $K$ and two powers of $C$. The first is

$$
\sum_{J_{1}, J_{2}}(-1)^{J_{1}-2 j_{\varphi}}(-1)^{J_{2}-2 j_{\varphi}} C\left(J_{1}\right) C\left(J_{2}\right) K\left(J_{1}, J_{2}, J^{\prime}\right)=(-1)^{J^{\prime}-2 j_{\varphi}} C\left(J^{\prime}\right)\left(C\left(J^{\prime}\right)+1\right)
$$

For a proof, consider the product of generators

$$
\left\{\tilde{\tau}^{a}, \tilde{\tau}^{b}\right\}_{i r}\left\{\tilde{\tau}^{a}, \tilde{\tau}^{b}\right\}_{k l}=2 \sum_{m n}\left(\tilde{\tau}_{i m}^{a} \tilde{\tau}_{m r}^{b} \tilde{\tau}_{k n}^{a} \tilde{\tau}_{n l}^{b}+\tilde{\tau}_{i m}^{a} \tilde{\tau}_{m r}^{b} \tilde{\tau}_{k n}^{b} \tilde{\tau}_{n l}^{a}\right)
$$

Using $\mathrm{SU}(2)$ commutation relations and eq. (4.4), it is easy to see that this becomes

$$
\sum_{a b}\left\{\tilde{\tau}^{a}, \tilde{\tau}^{b}\right\}_{i r}\left\{\tilde{\tau}^{a}, \tilde{\tau}^{b}\right\}_{k l}=2 \sum_{J M} C(J)(2 C(J)+1) \Sigma_{i k}^{(J), M} \Sigma_{r l}^{(J), M},
$$

but it is also expressed as

$$
\begin{aligned}
2 & \sum_{J_{1}, J_{2}} \sum_{M_{1}, M_{2}} \sum_{m, n} C\left(J_{1}\right) C\left(J_{2}\right) \\
& \times\left(\Sigma_{i k}^{\left(J_{1}\right), M_{1}} \Sigma_{m n}^{\left(J_{1}\right), M_{1}} \Sigma_{m n}^{\left(J_{2}\right), M_{2}} \Sigma_{r l}^{\left(J_{2}\right), M_{2}}+\Sigma_{i n}^{\left(J_{1}\right), M_{1}} \Sigma_{m l}^{\left(J_{1}\right), M_{1}} \Sigma_{m k}^{\left(J_{2}\right), M_{2}} \Sigma_{r n}^{\left(J_{2}\right), M_{2}}\right) \\
= & 2 \sum_{J_{1}, M_{1}} C\left(J_{1}\right)^{2} \Sigma_{i k}^{\left(J_{1}\right), M_{1}} \Sigma_{r l}^{\left(J_{1}\right), M_{1}} \\
& +2 \sum_{J_{1}, J_{2}, J_{3}} \sum_{M_{3}}(-1)^{J_{1}+J_{2}+J_{3}-6 j_{\varphi}} C\left(J_{1}\right) C\left(J_{2}\right) K\left(J_{1}, J_{2}, J_{3}\right) \Sigma_{i k}^{\left(J_{3}\right), M_{3}} \Sigma_{r l}^{\left(J_{3}\right), M_{3}}
\end{aligned}
$$

Equating these two expressions, multiplying both sides by $\sum_{M^{\prime}} \Sigma_{i k}^{\left(J^{\prime}\right), M^{\prime}} \Sigma_{r l}^{\left(J^{\prime}\right), M^{\prime}}$ and summing over $i, k, r, n$ yields eq. (4.65). A variant of this relation involving only one phase factor reads

$$
\sum_{J_{1}, J_{2}}(-1)^{J_{1}-2 j_{\varphi}} C\left(J_{1}\right) C\left(J_{2}\right) K\left(J_{1}, J_{2}, J_{3}\right)=C\left(J_{3}\right)\left(\mathcal{J}\left(j_{\varphi}\right)-1\right)
$$


To prove it, we perform the sum over $J_{2}$ using eq. (4.56) and the fact that $K$ is symmetric in its first two indices to find

$$
\begin{aligned}
& \sum_{J_{1}, J_{2}}(-1)^{J_{1}-2 j_{\varphi}} C\left(J_{1}\right) C\left(J_{2}\right) K\left(J_{1}, J_{2}, J_{3}\right) \\
& =\sum_{J_{1}}(-1)^{J_{1}-2 j_{\varphi}} \frac{\mathcal{D}\left(J_{1}\right) C\left(J_{1}\right)^{2} C\left(J_{3}\right)}{\mathcal{J}\left(j_{\varphi}\right) \mathcal{D}\left(j_{\varphi}\right)}=C\left(J_{3}\right)\left(\mathcal{J}\left(j_{\varphi}\right)-1\right),
\end{aligned}
$$

where in the last equality we used eq. (4.39). The relation without phase factors

$$
\sum_{J_{1}, J_{2}} C\left(J_{1}\right) C\left(J_{2}\right) K\left(J_{1}, J_{2}, J_{3}\right)=\frac{1}{3} \mathcal{J}\left(j_{\varphi}\right) \mathcal{D}\left(j_{\varphi}\right) C\left(J_{3}\right)
$$

is shown similar to the above, performing the sum over $J_{2}$ and using eq. (4.36).

Finally, we prove the following symmetry relation for a contraction of two $K$ factors:

$$
\sum_{J_{4}} K\left(J_{1}, J_{2}, J_{4}\right) K\left(J_{4}, J_{3}, J_{5}\right)=\sum_{J_{4}} K\left(J_{1}, J_{3}, J_{4}\right) K\left(J_{4}, J_{2}, J_{5}\right)
$$

First, consider the sum

$$
\sum_{i m s l} \sum_{L M N} \Sigma_{i m}^{\left(J_{1}\right), L} \Sigma_{s l}^{\left(J_{1}\right), L} \Sigma_{q s}^{\left(J_{2}\right), M} \Sigma_{r i}^{\left(J_{2}\right), M} \Sigma_{n m}^{\left(J_{3}\right), N} \Sigma_{k l}^{\left(J_{3}\right), N}
$$

This simplifies to

$$
\begin{aligned}
\sum_{J_{4}} \sum_{l m} \sum_{M N} K\left(J_{1}, J_{2}, J_{4}\right) \Sigma_{n m}^{\left(J_{3}\right), N} \Sigma_{k l}^{\left(J_{3}\right), N} \Sigma_{m q}^{\left(J_{4}\right), M} \Sigma_{l r}^{\left(J_{4}\right), M} \\
=\sum_{J_{4}, J_{5}} \sum_{M} K\left(J_{1}, J_{2}, J_{4}\right) K\left(J_{4}, J_{3}, J_{5}\right) \Sigma_{n r}^{\left(J_{5}\right), M} \Sigma_{k q}^{\left(J_{5}\right), M} .
\end{aligned}
$$

However, eq. (4.73) also reduces in a different way

$$
\begin{aligned}
\sum_{J_{4}} \sum_{i s} \sum_{M N} K\left(J_{1}, J_{3}, J_{4}\right) \Sigma_{q s}^{\left(J_{2}\right), N} \Sigma_{r i}^{\left(J_{2}\right), N} \Sigma_{i k}^{\left(J_{4}\right), M} \Sigma_{s n}^{\left(J_{4}\right), M} \\
=\sum_{J_{4}, J_{5}} \sum_{M} K\left(J_{1}, J_{3}, J_{4}\right) K\left(J_{4}, J_{2}, J_{5}\right) \Sigma_{n r}^{\left(J_{5}\right), M} \Sigma_{k q}^{\left(J_{5}\right), M} .
\end{aligned}
$$

Equating these two expressions gives the final result (4.72).

\section{$5 \quad$ Numerics}

In this section, we present numerical results for the running of the scalar and gauge couplings. All the numerical inputs are taken from ref. [27], see table 1. We employ the expressions given in ref. [28] to determine the initial conditions for the strong coupling $g_{s}\left(M_{Z}\right)=1.1626$, the top Yukawa coupling $y_{t}\left(M_{Z}\right)=0.9320$, and the quartic Higgs coupling $\lambda_{H}\left(M_{Z}\right)=0.5040$. We determine $g_{1}\left(M_{Z}\right)$ and $g_{2}\left(M_{Z}\right)$ directly via the relation

$$
\sin ^{2} \theta_{w}(\mu) \equiv \frac{g_{1}^{2}(\mu)}{g_{1}^{2}(\mu)+g_{2}^{2}(\mu)}
$$



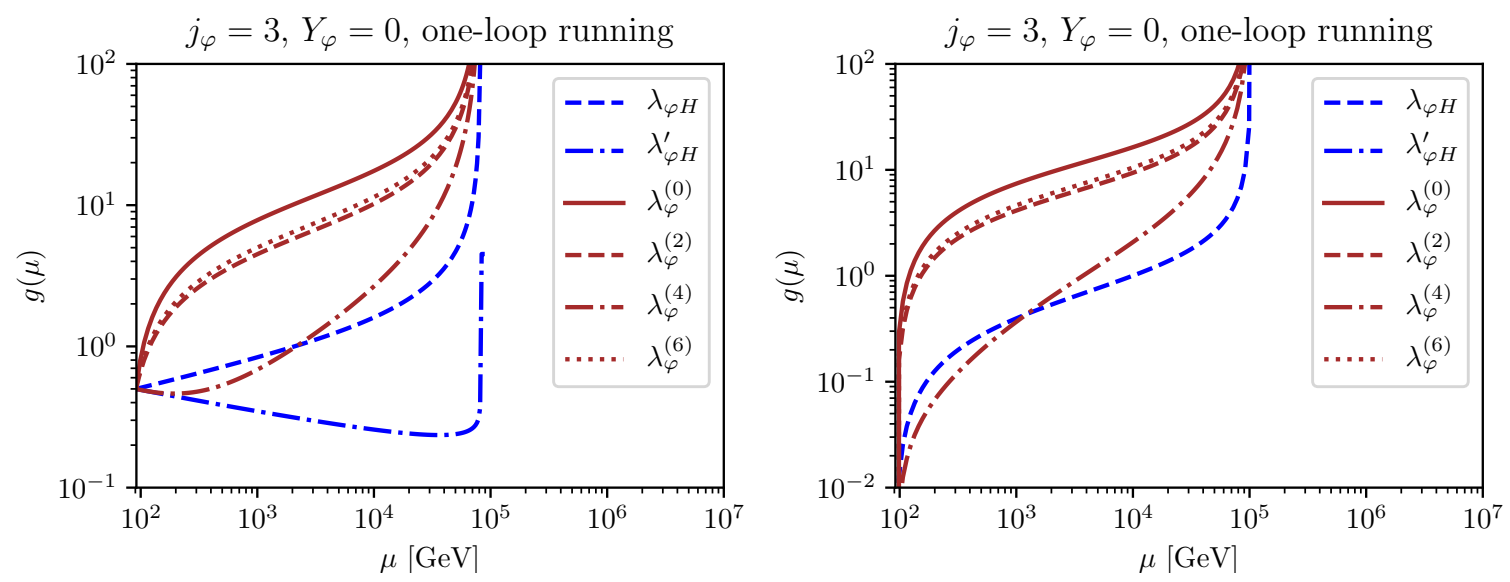

Figure 5. One-loop running of scalar quartic couplings for $j_{\varphi}=3$ with $Y_{\varphi}=0$ ("Minimal Scalar Dark Matter"). The dashed and dash-dotted lines denote the Higgs-portal couplings $\lambda_{\varphi H}$ and $\lambda_{\varphi H}^{\prime}$, respectively, while the solid lines denote the four scalar couplings $\lambda_{\varphi}^{(J)}$, for $J=0,2,4,6$. The running at one-loop exhibits a Landau pole around $\mu=10^{5} \mathrm{GeV}$. Left panel: all initial conditions are set to 0.5 at $\mu=M_{Z}$. Right panel: vanishing initial conditions at $\mu=M_{Z}$.

\begin{tabular}{|l|l|l|}
\hline$m_{t}($ pole $)=172.4(7) \mathrm{GeV}$ & $m_{b}\left(m_{b}\right)=4.18_{-0.02}^{+0.03} \mathrm{GeV}$ & $m_{c}\left(m_{c}\right)=1.27(2) \mathrm{GeV}$ \\
\hline$m_{\tau}=1.77686(12) \mathrm{GeV}$ & $M_{h}=125.10(14) \mathrm{GeV}$ & $M_{Z}=91.1876(21) \mathrm{GeV}$ \\
\hline$\alpha^{(5)}\left(M_{Z}\right)^{-1}=127.952(9)$ & $\sin ^{2} \theta\left(M_{Z}\right)=0.23121(4)$ & $\alpha_{s}\left(M_{Z}\right)=0.1179(10)$ \\
\hline$G_{F}=1.11663787(6) \times 10^{-5} \mathrm{GeV}^{-2}$ & & \\
\hline
\end{tabular}

Table 1. Numerical input used to determine the initial conditions of the coupling constants. All values are taken from ref. [27].

to find $g_{1}\left(M_{Z}\right)=0.3574, g_{2}\left(M_{Z}\right)=0.6517$. To determine $y_{\tau}\left(M_{Z}\right)=0.0102$ we used $m_{\tau}=1.77686(12) \mathrm{GeV}$, and the relations

$$
y_{\tau}=\frac{\sqrt{2} m_{\tau}}{v_{\mathrm{EW}}}, \quad G_{F}=\frac{1}{\sqrt{2} v_{\mathrm{EW}}^{2}} .
$$

Note that $G_{F}$ is RG invariant, and we neglect the QED running of $m_{\tau}$. We obtain $y_{c}\left(M_{Z}\right)=0.0036$ and $y_{b}\left(M_{Z}\right)=0.0164$ in the six-flavor theory by four-loop QCD running and decoupling of the corresponding quark masses and subsequent conversion using an expression analogous to eq. (5.2). As we are only interested in the qualitative behaviour of our results, we neglect uncertainties throughout. We solve the coupled system of RG equations numerically, using the python package pywigxjpf [29] and the Mathematica code found in ref. [30] for the numerical evaluation of the Wigner $9 j$ symbols.

In figure 5 we show the one-loop running of all scalar couplings for $j_{\varphi}=3$, with scalar hypercharge $Y_{\varphi}=0$. This case corresponds to the "minimal scalar dark matter" (MSDM) scenario in ref. [31], amended by the two Higgs-portal couplings $\lambda_{\varphi H}$ and $\lambda_{\varphi H}^{\prime}$. In the left panel, we assumed an initial condition of $\lambda_{i}\left(M_{Z}\right)=0.5$ for all four scalar couplings and the two Higgs-portal couplings. The high-energy behaviour is largely independent of 

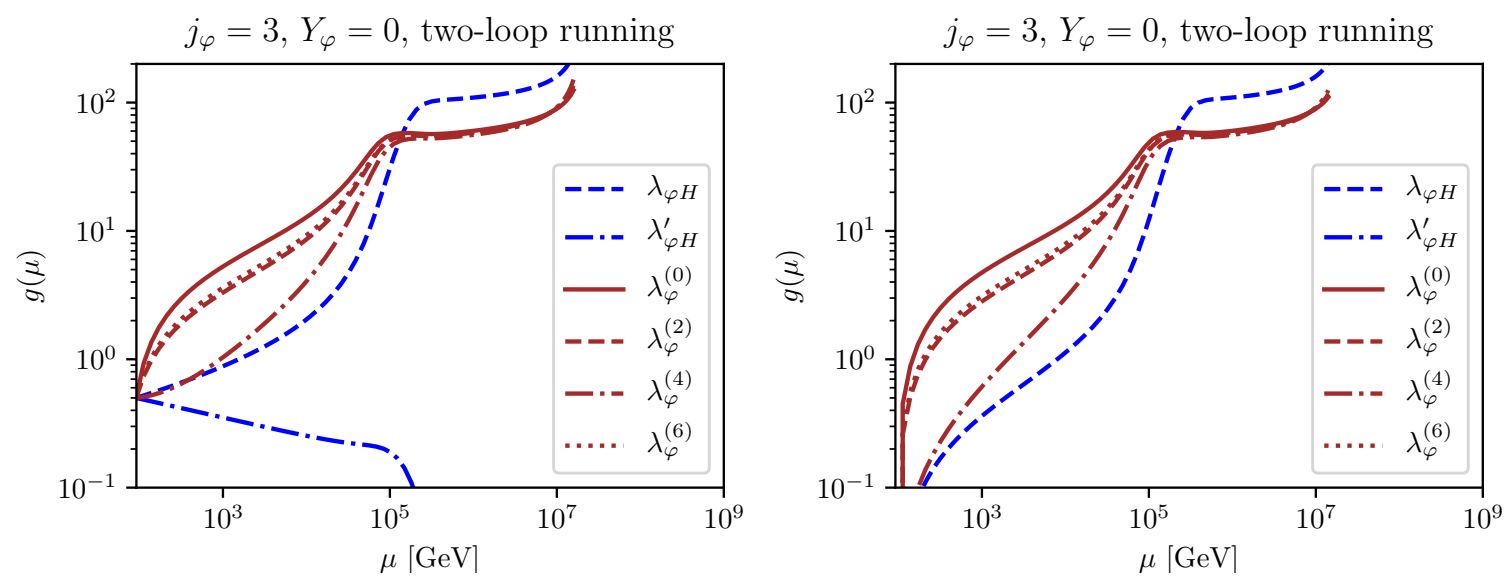

Figure 6. Two-loop running of scalar quartic couplings for $j_{\varphi}=3$ with $Y_{\varphi}=0$. The notation is the same as in figure 5 .
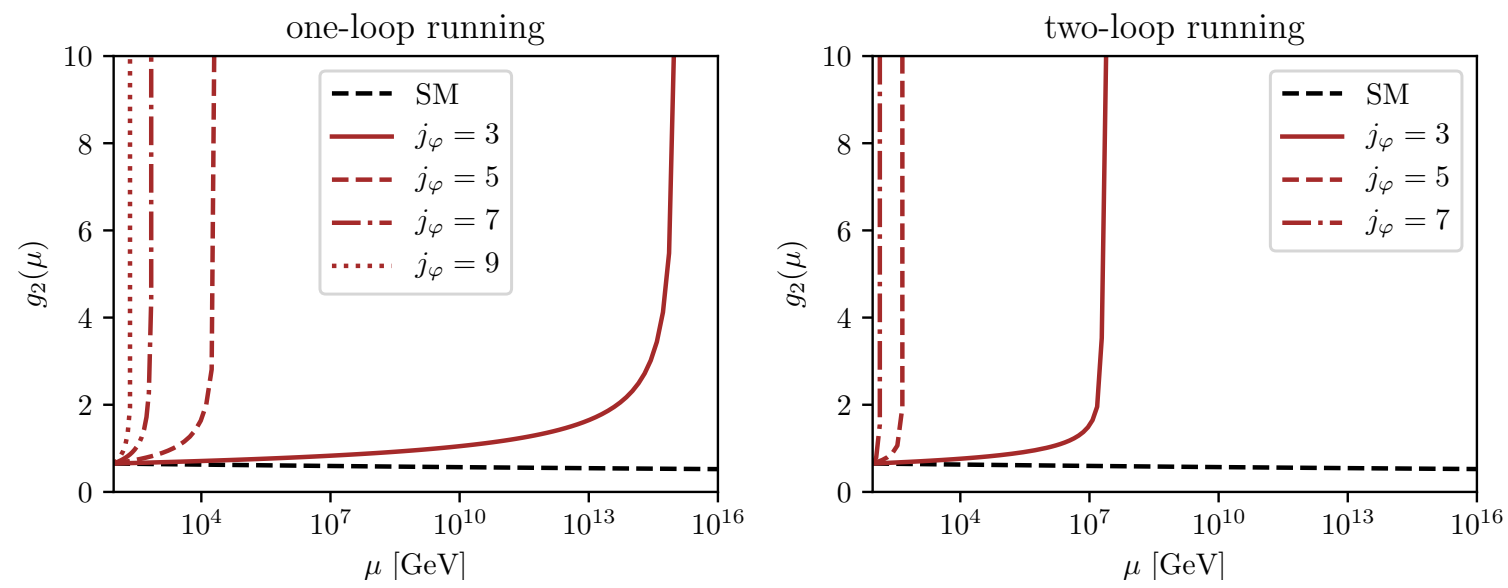

Figure 7. Running of the SU(2) gauge coupling $g_{2}$ at one-loop (left panel) and two-loop (right panel), for $Y_{\varphi}=0$. The black dashed line shows the SM result. The brown lines correspond to different representations of the complex scalar. Here, we assumed vanishing initial conditions for all non-SM scalar couplings at $\mu=M_{Z}$.

these assumptions; in fact, even if the couplings are all zero at the weak scale, large values get generated via weak gauge-boson exchange (with the exception of $\lambda_{\varphi H}^{\prime}$ ). The couplings quickly enter a non-perturbative regime and run into a Landau pole around $10^{5} \mathrm{GeV}$.

Next, we study the impact of the two-loop corrections to the RG evolution of the scalar couplings in the same scenario, see figure 6. Again, we display the results for the two sets of initial conditions. Note that the Landau pole around $\mu=10^{5} \mathrm{GeV}$ is shifted to the higher scale $\mu=10^{7} \mathrm{GeV}$, with a plateau-like behaviour in between. However, these features appear at non-perturbative values for the coupling constants and should therefore not be taken too literally. The only significant change is that the "triplet" Higgs-portal coupling $\lambda_{\varphi H}^{\prime}$ turns out to be asymptotically free.

Finally, we examine the impact of the new scalar degrees of freedom on the running of the SM couplings. We keep assuming vanishing hypercharge for the new scalars, $Y_{\varphi}=0$, 

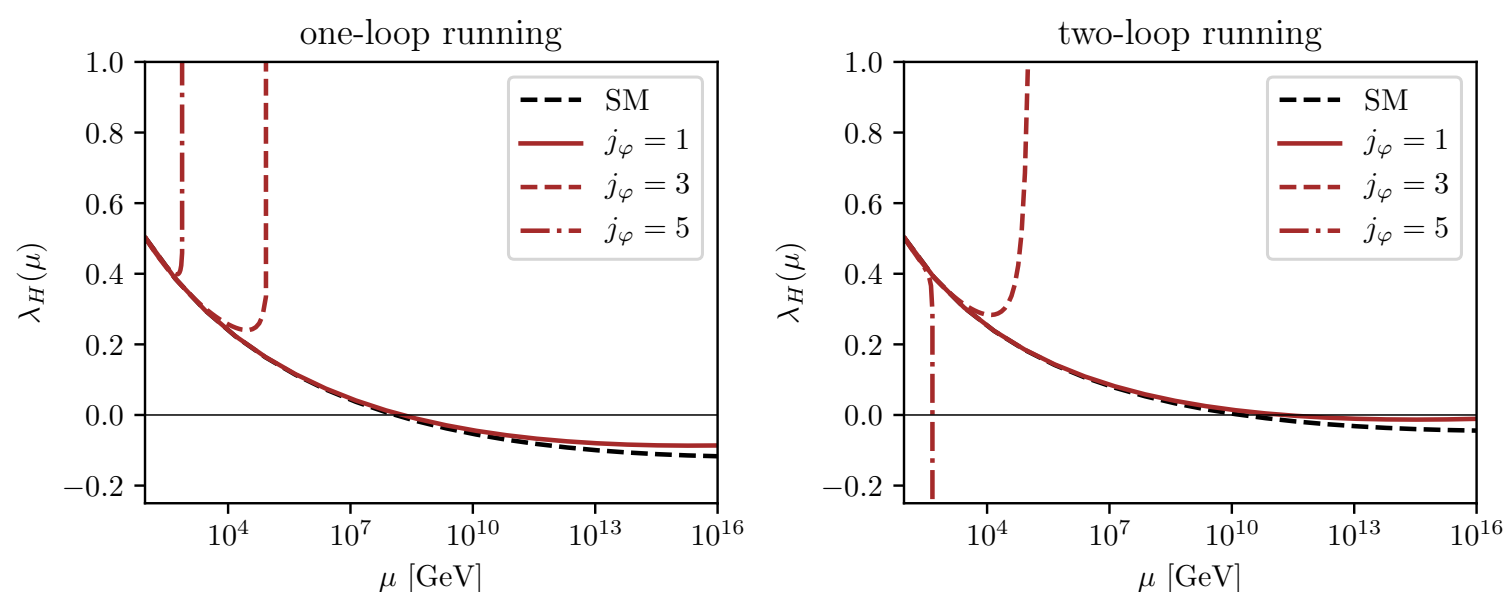

Figure 8. Running of the quartic Higgs coupling $\lambda_{H}$ at one-loop (left panel) and two-loop (right panel), for $Y_{\varphi}=0$. The black dashed line shows the SM result. The brown lines correspond to different representations of the complex scalar. Here, we assumed vanishing initial conditions for all non-SM scalar couplings at $\mu=M_{Z}$.

and focus on the evolution on the gauge coupling $g_{2}$ first. The running of $g_{2}$ is displayed in figure 7. In the left panel, we show the one-loop evolution. We see that, at one-loop, the $\mathrm{SU}(2)$ gauge coupling exhibits a Landau pole at around $10^{15} \mathrm{GeV}$ for $j_{\varphi}=3$ (MSDM), while for higher representations the Landau pole appears close to or below the TeV scale. This behaviour has been qualitatively described in, for instance, ref. [8]. Looking at the two-loop results in the right panel in figure 7 , we see that the Landau pole for $j_{\varphi}=3$ is significantly shifted down to $10^{7} \mathrm{GeV}$, while all other poles lie below the $\mathrm{TeV}$ scale. Apparently, the SM extended by MSDM cannot be perturbative up to the Planck scale.

As our last example, we show the evolution of the quartic Higgs coupling in figure 8. Again we display the one-loop results in the left panel, and the two-loop results in the right panel. While the SM evolution of $\lambda_{H}$ (black dashed line) is only marginally affected by the presence of an additional scalar multiplet with $j_{\varphi}=1$, higher representations lead to a drastic departure from this picture. For $j_{\varphi}=3$ (MSDM), the Higgs quartic runs into a Landau pole around $10^{5} \mathrm{GeV}$, while the pole lies at the $\mathrm{TeV}$ scale for $j_{\varphi}=5$. Interestingly, the two-loop results show that this pole is in fact negative.

We relegate a more detailed discussion of the phenomenological implications of these results to future work.

\section{Conclusions}

In this work, we constructed the form of the potential involving four-point interaction of a complex scalar field furnishing a general irreducible representation of the electroweak gauge group $\mathrm{SU}(2) \times \mathrm{U}(1)$, in terms of Clebsch-Gordan coefficients. We presented the beta functions determining the RG evolution of the scalar as well as the SM couplings explicitly in terms of $\mathrm{SU}(2)$ group invariants, up to the two-loop level. As an important ingredient of our calculation we proved a set of algebraic relations that we used to express the results for 
the one- and two-loop Green's functions in terms of our basis operators. For convenience, auxiliary files containing the analytic results of the beta functions in the form of a python module, as well as a mathematica package, are available at

https://gitlab.com/complex-beta-function .

Our results are completely general and might have applications in many fields. As one example, we studied the RG flow of the self interactions of scalar dark matter in minimal dark matter models [1], and the impact of the scalar fields on the RG evolution of the SM couplings. Moreover, the beta functions will be a necessary ingredient in the RG analysis of scalar dark matter interacting via higher dimension operators [3, 4].

A generalization of our results in this direction would be to consider the self interactions of fermionic dark matter. This case is more complicated since the interactions start at mass dimension six, and additional Fierz relations associated with the Dirac-matrix structure restrict the form of all possible operators. This investigation is relegated to future work.

\section{Acknowledgments}

We thank Emmanuel Stamou and Jure Zupan for suggestions and comments on the manuscript, and Jared Evans and Florian Hanisch for discussions. JB acknowledges support in part by DOE grant DE-SC0020047.

\section{A Analytic checks of our calculation}

As a check of our results we used a generalized $R_{\xi}$ gauge for the $W, B$ and $G$ fields and verified that all beta functions are gauge-parameter independent. For completeness, we provide here the gauge-fixing and ghost terms in our Lagrangian:

$$
\begin{aligned}
\mathcal{L}_{\text {gf }}+\mathcal{L}_{\text {ghost }}= & -\frac{1}{2 \xi_{W}}\left(\partial_{\mu} W^{a \mu}\right)^{2}-\frac{1}{2 \xi_{B}}\left(\partial_{\mu} B^{\mu}\right)^{2}-\frac{1}{2 \xi_{G}}\left(\partial_{\mu} G^{A \mu}\right)^{2} \\
& +\partial_{\mu} \bar{u}_{W}^{a} \partial^{\mu} u_{W}^{a}+g_{2} \epsilon^{a b c}\left(\partial^{\mu} \bar{u}_{W}^{a}\right) W_{\mu}^{b} u_{W}^{c} \\
& +\partial_{\mu} \bar{u}_{G}^{A} \partial^{\mu} u_{G}^{A}-g_{s} f^{A B C}\left(\partial^{\mu} \bar{u}_{G}^{A}\right) G_{\mu}^{B} u_{G}^{C} .
\end{aligned}
$$

As a second consistency check of our calculation, we verified that all two-loop counterterms are local, i.e. they do not contain any explicit logarithms of the renormalization scale $\mu$. As a third check of our calculation, we derive the explicit expressions of the beta function in terms of the coupling counterterms (see below). The finiteness of the beta function as $\epsilon \rightarrow 0$ yields consistency relations that allow to calculate the quadratic pole of the two-loop coupling renormalization constants in terms of the one-loop results. These quadratic poles are in full agreement with the results of our calculcation. For completeness, we provide the expressions for the quadratic poles in appendix B.

In the remainder of this section, we derive the relation between the beta function and the residua of the coupling renormalization constants, as well as the relation between the linear one-loop poles and the quadratic two-loop poles. As above, we denote the all couplings generically by a coupling vector $g_{i}$. The bare couplings $g_{i, 0}$ are expressed in terms 
of the renormalized couplings $g_{i}$ as $g_{i, 0}=\mu^{a_{i} \epsilon} Z_{g_{i}} g_{i}$, where $a_{i}=1$ if $g_{i}$ is a gauge or Yukawa coupling, and $a_{i}=2$ if $g_{i}$ is a scalar coupling (the coefficients $a_{i}$ are chosen such that all couplings remain dimensionless in $d$ space-time dimensions). Here, $\mu$ is the renormalization scale, and the $Z_{g_{i}}$ are the coupling renormalization constants. We expand the $Z_{g_{i}}$ by order of pole as

$$
Z_{g_{i}}=1+\sum_{l=1}^{\infty} \frac{1}{\epsilon^{l}} Z_{g_{i}, l}
$$

and use standard methods [32] to express the beta function in terms of the derivatives of the linear poles of the coupling counterterms:

$$
\beta_{i}\left(g_{j}, \epsilon\right)=-a_{i} g_{i} \epsilon+g_{i} \sum_{k} a_{k} g_{k} \frac{\partial Z_{g_{i}, 1}}{\partial g_{k}} .
$$

The fact that the $1 / \epsilon$ contributions to the beta function have to cancel leads to the following consistency condition on the counterterms:

$$
\sum_{k} a_{k} g_{k} \frac{\partial Z_{g_{i}, 2}}{\partial g_{k}}=\sum_{k} a_{k} g_{k} Z_{g_{i}, 1} \frac{\partial Z_{g_{i}, 1}}{\partial g_{k}}+\sum_{k m} a_{k} g_{k} g_{m} \frac{\partial Z_{g_{m}, 1}}{\partial g_{k}} \frac{\partial Z_{g_{i}, 1}}{\partial g_{m}}
$$

Further conditions can be derived by requiring the cancelation of the higher poles; however, they do not lead to additional constraints on the two-loop counterterms. The relation (A.4) is made more explicit by expanding the counterterms by loop-order,

$$
Z_{g_{i}}=1+\sum_{n} \delta Z_{g_{i}}^{(n)}=1+\sum_{n} \sum_{l} \frac{1}{\epsilon^{l}} \delta Z_{g_{i}, l}^{(n)} .
$$

Keeping only terms at two-loop order, and using the fact that the counterterms are polynomials in the couplings, we arrive at

$$
\sum_{k} a_{k} g_{k} \frac{\partial\left(\delta Z_{g_{i}, 2}^{(2)}\right)}{\partial g_{k}}=4 \delta Z_{g_{i}, 2}^{(2)}, \quad \sum_{k} a_{k} g_{k} \frac{\partial\left(\delta Z_{g_{i}, 2}^{(1)}\right)}{\partial g_{k}}=2 \delta Z_{g_{i}, 2}^{(1)} .
$$

We then rewrite eq. (A.4) as

$$
\delta Z_{g_{i}, 2}^{(2)}=\frac{1}{2}\left(\delta Z_{g_{i}, 1}^{(1)}\right)^{2}+\frac{1}{2} \sum_{k} g_{k} \delta Z_{g_{k}, 1}^{(1)} \frac{\partial\left(\delta Z_{g_{i}, 1}^{(1)}\right)}{\partial g_{k}} .
$$

We checked explicitly that this relation is satisfied for all our coupling counterterms.

\section{B Renormalization constants}

In this appendix we collect all renormalization constants that were needed in intermediate steps of the calculation, namely, all field and artificial-mass counterterms. The $1 / \epsilon$ pole parts of the coupling counterterms give rise to the beta functions, as explained in appendix A, and are not repeated here. For completeness, however, we show the $1 / \epsilon^{2}$ pole 
parts. The $\overline{\mathrm{MS}}$ scheme is used throughout. For the one-loop field renormalization constants we find

$$
\begin{aligned}
\delta Z_{\varphi}^{(1)} & =\frac{g_{1}^{2}}{16 \pi^{2} \epsilon} \frac{Y_{\varphi}^{2}}{4}\left(3-\xi_{B}\right)+\frac{g_{2}^{2}}{16 \pi^{2} \epsilon} \mathcal{J}\left(j_{\varphi}\right)\left(3-\xi_{W}\right) \\
\delta Z_{H}^{(1)} & =\frac{g_{1}^{2}}{16 \pi^{2} \epsilon} \frac{1}{4}\left(3-\xi_{B}\right)+\frac{g_{2}^{2}}{16 \pi^{2} \epsilon} \frac{3}{4}\left(3-\xi_{W}\right)-\frac{1}{16 \pi^{2} \epsilon}\left[3\left(y_{t}^{2}+y_{b}^{2}+y_{c}^{2}\right)+y_{\tau}^{2}\right] \\
\delta Z_{B}^{(1)} & =-\frac{g_{1}^{2}}{16 \pi^{2} \epsilon}\left(\frac{Y_{\varphi}^{2}}{12} \mathcal{D}\left(j_{\varphi}\right)+\frac{20}{9} n_{g}+\frac{1}{6}\right) \\
\delta Z_{W}^{(1)} & =\frac{g_{2}^{2}}{16 \pi^{2} \epsilon}\left(\frac{13}{3}-\frac{1}{6}-\xi_{W}-\frac{1}{9} \mathcal{J}\left(j_{\varphi}\right) \mathcal{D}\left(j_{\varphi}\right)-\frac{4}{3} n_{g}\right) \\
\delta Z_{G}^{(1)} & =\frac{g_{s}^{2}}{16 \pi^{2} \epsilon}\left(\frac{13}{2}-\frac{3}{2} \xi_{G}-n_{g}\right) \\
\delta Z_{u_{W}}^{(1)} & =\frac{g_{2}^{2}}{16 \pi^{2} \epsilon} \frac{1}{2}\left(3-\xi_{W}\right) \\
\delta Z_{Q_{L, i}}^{(1)} & =-\frac{g_{1}^{2}}{16 \pi^{2} \epsilon} \frac{\xi_{B}}{36}-\frac{g_{2}^{2}}{16 \pi^{2} \epsilon} \frac{3 \xi_{W}}{4}-\frac{g_{s}^{2}}{16 \pi^{2} \epsilon} \frac{4 \xi_{G}}{3}-\frac{1}{16 \pi^{2} \epsilon}\left(\frac{y_{u_{i}}^{2}}{2}+\frac{y_{d_{i}}^{2}}{2}\right) \\
\delta Z_{u_{R, i}}^{(1)} & =-\frac{g_{1}^{2}}{16 \pi^{2} \epsilon} \frac{4 \xi_{B}}{9}-\frac{g_{s}^{2}}{16 \pi^{2} \epsilon} \frac{4 \xi_{G}}{3}-\frac{y_{u_{i}}^{2}}{16 \pi^{2} \epsilon} \\
\delta Z_{d_{R, i}}^{(1)} & =-\frac{g_{1}^{2}}{16 \pi^{2} \epsilon} \frac{\xi_{B}}{9}-\frac{g_{s}^{2}}{16 \pi^{2} \epsilon} \frac{4 \xi_{G}}{3}-\frac{y_{d_{i}}^{2}}{16 \pi^{2} \epsilon} \\
\delta Z_{L_{L, i}}^{(1)} & =-\frac{g_{1}^{2}}{16 \pi^{2} \epsilon} \frac{\xi_{B}}{4}-\frac{g_{2}^{2}}{16 \pi^{2} \epsilon} \frac{3 \xi_{W}}{4}-\frac{1}{16 \pi^{2} \epsilon} \frac{y_{\ell_{i}}^{2}}{2} \\
\delta Z_{\ell_{R, i}}^{(1)} & =-\frac{g_{1}^{2}}{16 \pi^{2} \epsilon} \xi_{B}-\frac{y_{\ell_{i}}^{2}}{16 \pi^{2} \epsilon} \cdot
\end{aligned}
$$

At one-loop, the artificial-mass counterterms are

$$
\begin{aligned}
\delta Z_{M_{\mathrm{IRA}}^{2}, \varphi}^{(1)} & =\frac{1}{16 \pi^{2} \epsilon} \sum_{J}^{\prime} \lambda^{(J)} \frac{\mathcal{D}(J)}{\mathcal{D}\left(j_{\varphi}\right)}+\frac{1}{16 \pi^{2} \epsilon} \frac{\lambda_{\varphi H}}{2} \\
\delta Z_{M_{\mathrm{IRA}}^{2}, H}^{(1)} & =\frac{\lambda_{\varphi H}}{16 \pi^{2} \epsilon} \frac{\mathcal{D}\left(j_{\varphi}\right)}{4}+\frac{\lambda_{H}}{16 \pi^{2} \epsilon} \frac{3}{2}-\frac{3}{16 \pi^{2} \epsilon}\left[3\left(y_{t}^{2}+y_{b}^{2}+y_{c}^{2}\right)+y_{\tau}^{2}\right] \\
\delta Z_{M_{\mathrm{IRA}}^{2}, B}^{(1)} & =\frac{g_{1}^{2}}{16 \pi^{2} \epsilon}\left(\frac{Y_{\varphi}^{2}}{12} \mathcal{D}\left(j_{\varphi}\right)-\frac{40}{9} n_{g}+\frac{1}{6}\right) \\
\delta Z_{M_{\mathrm{IRA}}^{2}, W}^{(1)} & =\frac{g_{2}^{2}}{16 \pi^{2} \epsilon}\left(\frac{1}{9} \mathcal{J}\left(j_{\varphi}\right) \mathcal{D}\left(j_{\varphi}\right)-\frac{1}{2} \xi_{W}-\frac{29}{6}-\frac{8}{3} n_{g}+\frac{1}{6}\right), \\
\delta Z_{M_{\mathrm{IRA}}^{2}, G}^{(1)} & =\frac{g_{s}^{2}}{16 \pi^{2} \epsilon}\left(-\frac{3}{4}-\frac{9}{4} \xi_{G}-3 n_{g}\right) \\
\delta Z_{M_{\mathrm{IRA}}^{2}, u_{W}}^{(1)} & =\frac{g_{2}^{2}}{16 \pi^{2} \epsilon} \frac{1}{2}\left(\xi_{W}-3\right) .
\end{aligned}
$$


We find the following quadratic poles for the two-loop contributions to the scalar and gauge coupling counterterms:

$$
\begin{aligned}
\delta Z_{g_{1}}^{(2)} & =\frac{g_{1}^{4}}{\left(16 \pi^{2}\right)^{2} \epsilon^{2}}\left(\frac{Y_{\varphi}^{4}}{384} \mathcal{D}\left(j_{\varphi}\right)^{2}+\frac{Y_{\varphi}^{2}}{96} \mathcal{D}\left(j_{\varphi}\right)+\frac{5}{36} Y_{\varphi}^{2} \mathcal{D}\left(j_{\varphi}\right) n_{g}\right) \\
\delta Z_{g_{2}}^{(2)} & =\frac{g_{2}^{4}}{\left(16 \pi^{2}\right)^{2} \epsilon^{2}}\left(\frac{1}{216} \mathcal{D}\left(j_{\varphi}\right)^{2} \mathcal{J}\left(j_{\varphi}\right)^{2}+\frac{1}{72} \mathcal{D}\left(j_{\varphi}\right) \mathcal{J}\left(j_{\varphi}\right)\left(8 n_{g}-43\right)\right) \\
\delta Z_{y_{t}}^{(2)} & =-\frac{17}{576} \frac{g_{1}^{4}}{\left(16 \pi^{2}\right)^{2} \epsilon^{2}} \mathcal{D}\left(j_{\varphi}\right) Y_{\varphi}^{2}-\frac{1}{16} \frac{g_{2}^{4}}{\left(16 \pi^{2}\right)^{2} \epsilon^{2}} \mathcal{J}\left(j_{\varphi}\right) \mathcal{D}\left(j_{\varphi}\right), \\
\delta Z_{y_{b}}^{(2)} & =-\frac{5}{576} \frac{g_{1}^{4}}{\left(16 \pi^{2}\right)^{2} \epsilon^{2}} \mathcal{D}\left(j_{\varphi}\right) Y_{\varphi}^{2}-\frac{1}{16} \frac{g_{2}^{4}}{\left(16 \pi^{2}\right)^{2} \epsilon^{2}} \mathcal{J}\left(j_{\varphi}\right) \mathcal{D}\left(j_{\varphi}\right), \\
\delta Z_{y_{c}}^{(2)} & =-\frac{17}{576} \frac{g_{1}^{4}}{\left(16 \pi^{2}\right)^{2} \epsilon^{2}} \mathcal{D}\left(j_{\varphi}\right) Y_{\varphi}^{2}-\frac{1}{16} \frac{g_{2}^{4}}{\left(16 \pi^{2}\right)^{2} \epsilon^{2}} \mathcal{J}\left(j_{\varphi}\right) \mathcal{D}\left(j_{\varphi}\right), \\
\delta Z_{y_{\tau}}^{(2)} & =-\frac{5}{64} \frac{g_{1}^{4}}{\left(16 \pi^{2}\right)^{2} \epsilon^{2}} \mathcal{D}\left(j_{\varphi}\right) Y_{\varphi}^{2}-\frac{1}{16} \frac{g_{2}^{4}}{\left(16 \pi^{2}\right)^{2} \epsilon^{2}} \mathcal{J}\left(j_{\varphi}\right) \mathcal{D}\left(j_{\varphi}\right) \\
\lambda_{i}^{(J)} \delta Z_{\lambda_{i}^{(J)}}^{(2)} & =\frac{1}{\left(16 \pi^{2}\right)^{2} \epsilon^{2}}\left(+g_{1}^{6} \Lambda_{i, 60}^{(J)(2)}+g_{1}^{4} g_{2}^{2} \Lambda_{i, 42}^{(J)(2)}+g_{2}^{2} g_{2}^{4} \Lambda_{i, 24}^{(J)(2)}+g_{2}^{6} \Lambda_{i, 06}^{(J)(2)}\right. \\
& +g_{1}^{4} \Lambda_{i, 40}^{(J)(2)}+g_{1}^{2} g_{2}^{2} \Lambda_{i, 22}^{(J)(2)}+g_{2}^{4} \Lambda_{i, 04}^{(J)(2)} \\
& \left.+g_{1}^{2} \Lambda_{i, 20}^{(J)(2)}+g_{2}^{2} \Lambda_{i, 02}^{(J)(2)}+\Lambda_{i, 00}^{(J)(2)}\right)
\end{aligned}
$$

with $\lambda_{i}=\lambda_{\varphi}^{(J)}, \lambda_{\varphi H}, \lambda_{\varphi H}^{\prime}, \lambda_{H}$, and coefficients

$$
\begin{aligned}
\Lambda_{\varphi, 60}^{(J)(2)=} & Y_{\varphi}^{6}\left(\frac{\mathcal{D}\left(j_{\varphi}\right)}{16}-\frac{9}{16}\right)+Y_{\varphi}^{4}\left(\frac{1}{8}+\frac{5}{3} n_{g}\right) \\
\Lambda_{\varphi, 42}^{(J)(2)}= & Y_{\varphi}^{4}\left[\mathcal{J}\left(j_{\varphi}\right)\left(\frac{9}{4}-\frac{1}{4} \mathcal{D}\left(j_{\varphi}\right)\right)-\mathcal{J}(J)\left(\frac{9}{4}-\frac{1}{8} \mathcal{D}\left(j_{\varphi}\right)\right)\right] \\
& +Y_{\varphi}^{2}\left(\frac{1}{4}+\frac{10}{3} n_{g}\right)\left(\mathcal{J}(J)-2 \mathcal{J}\left(j_{\varphi}\right)\right) \\
\Lambda_{\varphi, 24}^{(J)(2)=} & Y_{\varphi}^{2}\left[\mathcal{J}\left(j_{\varphi}\right)\left(9 \mathcal{J}\left(j_{\varphi}\right)-\frac{1}{3} \mathcal{D}\left(j_{\varphi}\right) \mathcal{J}\left(j_{\varphi}\right)+26-4 n_{g}\right)\right. \\
& \left.-\mathcal{J}(J)\left(\frac{9}{4} \mathcal{J}(J)-\frac{1}{6} \mathcal{D}\left(j_{\varphi}\right) \mathcal{J}\left(j_{\varphi}\right)+13-2 n_{g}\right)\right] \\
\Lambda_{\varphi, 06}^{(J)(2)}= & \left(\frac{\mathcal{J}(J)}{2}-\mathcal{J}\left(j_{\varphi}\right)\right)\left(\frac{\mathcal{J}(J)}{2}-\mathcal{J}\left(j_{\varphi}\right)+\frac{1}{2}\right) \\
& \left.Y_{\varphi}^{4}\left(j_{\varphi}\right) \mathcal{D}\left(j_{\varphi}\right)-36 \mathcal{J}\left(j_{\varphi}\right)-86+16 n_{g}\right) \\
& \left.4 \lambda_{\varphi}^{(J)}-\frac{\mathcal{D}\left(j_{\varphi}\right)}{4} \lambda_{\varphi}^{(J)}+3 \sum_{J_{1}}^{\prime} \lambda_{\varphi}^{\left(J_{1}\right)} \frac{\mathcal{D}\left(J_{1}\right)}{\mathcal{D}\left(j_{\varphi}\right)}\right)-Y_{\varphi}^{2}\left(\frac{1}{8}+\frac{5}{3} n_{g}\right)+\frac{3 \lambda_{\varphi} H}{8} Y_{\varphi}^{2}, \\
\Lambda_{\varphi, 40}^{(J)(2)}= & \\
\Lambda_{\varphi, 22}^{(J)(2)}= & Y_{\varphi}^{2} \sum_{J_{1}}^{\prime} \lambda_{\varphi}^{\left(J_{1}\right)} \frac{\mathcal{D}\left(J_{1}\right)}{\mathcal{D}\left(j_{\varphi}\right)}\left[\frac{3}{2} \frac{\mathcal{J}\left(J_{1}\right) \mathcal{J}(J)}{\mathcal{J}\left(j_{\varphi}\right)}-3\left(\mathcal{J}\left(J_{1}\right)+\mathcal{J}(J)\right)+6 \mathcal{J}\left(j_{\varphi}\right)\right] \\
& +Y_{\varphi}^{2} \lambda_{\varphi}^{(J)}\left(6 \mathcal{J}\left(j_{\varphi}\right)+3 \mathcal{J}(J)\right)+\frac{3}{8} Y_{\varphi} \lambda_{\varphi H}^{\prime}\left(\mathcal{J}(J)-2 \mathcal{J}\left(j_{\varphi}\right)\right),
\end{aligned}
$$




$$
\begin{aligned}
& \Lambda_{\varphi, 04}^{(J)(2)}=\lambda_{\varphi}^{(J)}\left[\mathcal{J}\left(j_{\varphi}\right)\left(\frac{37}{2}+36 \mathcal{J}\left(j_{\varphi}\right)-4 n_{g}-\frac{1}{3} \mathcal{D}\left(j_{\varphi}\right) \mathcal{J}\left(j_{\varphi}\right)\right)+\mathcal{J}(J)\left(3+\frac{3}{2} \mathcal{J}(J)-6 \mathcal{J}\left(j_{\varphi}\right)\right)\right] \\
& +\sum_{J_{1}, J_{2}}^{\prime} K\left(J_{1}, J_{2}, J\right) \lambda_{\varphi}^{\left(J_{2}\right)}\left[6 \mathcal{J}\left(J_{1}\right)^{2}-24 \mathcal{J}\left(j_{\varphi}\right) \mathcal{J}\left(J_{1}\right)\right]+\frac{3 \lambda_{\varphi} H}{2} \mathcal{J}\left(j_{\varphi}\right) \\
& +\sum_{J_{1}}^{\prime} \lambda_{\varphi}^{\left(J_{1}\right)} \frac{\mathcal{D}\left(J_{1}\right)}{\mathcal{D}\left(j_{\varphi}\right)}\left[6 \mathcal{J}\left(j_{\varphi}\right)+12 \mathcal{J}\left(j_{\varphi}\right)^{2}-3 \mathcal{J}(J)-3 \mathcal{J}\left(J_{1}\right)+\frac{3}{2} \frac{\mathcal{J}\left(J_{1}\right) \mathcal{J}(J)}{\mathcal{J}\left(j_{\varphi}\right)}\right], \\
& \Lambda_{\varphi, 20}^{(J)(2)}=-\frac{9 Y_{\varphi}^{2}}{8}\left(4 \sum_{J_{1}, J_{2}}^{\prime} K\left(J_{1}, J_{2}, J\right) \lambda_{\varphi}^{\left(J_{1}\right)} \lambda_{\varphi}^{\left(J_{2}\right)}+\left(\lambda_{\varphi}^{(J)}\right)^{2}\right) \\
& -\frac{3}{128}\left(\lambda_{\varphi H}^{\prime}\right)^{2}\left(\mathcal{J}(J)-2 \mathcal{J}\left(j_{\varphi}\right)\right)\left(1+2 Y_{\varphi}^{2}\right)-\frac{3 \lambda_{\varphi H}^{2}}{16}\left(1+2 Y_{\varphi}^{2}\right), \\
& \Lambda_{\varphi, 02}^{(J)(2)}=-\mathcal{J}\left(j_{\varphi}\right)\left[18 \sum_{J_{1}, J_{2}}^{\prime} K\left(J_{1}, J_{2}, J\right) \lambda_{\varphi}^{\left(J_{1}\right)} \lambda_{\varphi}^{\left(J_{2}\right)}+\frac{9}{2}\left(\lambda_{\varphi}^{(J)}\right)^{2}\right] \\
& -\frac{3}{16}\left(\lambda_{\varphi H}^{\prime}\right)^{2}\left(\mathcal{J}\left(j_{\varphi}\right)+\frac{3}{8}\right)\left(\mathcal{J}(J)-2 \mathcal{J}\left(j_{\varphi}\right)\right)-\frac{3 \lambda_{\varphi H}^{2}}{2}\left(\mathcal{J}\left(j_{\varphi}\right)+\frac{3}{8}\right), \\
& \Lambda_{\varphi, 00}^{(J)(2)}=\frac{\left(\lambda_{\varphi}^{(J)}\right)^{3}}{4}+\sum_{J_{1}, J_{2}}^{\prime} K\left(J_{1}, J_{2}, J\right) \lambda_{\varphi}^{\left(J_{1}\right)} \lambda_{\varphi}^{\left(J_{2}\right)}\left(\lambda_{\varphi}^{\left(J_{1}\right)}+\lambda_{\varphi}^{(J)}\right) \\
& +4 \sum_{J_{1}, J_{2}, J_{3}, J_{4}}{ }^{\prime} K\left(J_{1}, J_{2}, J_{3}\right) K\left(J_{3}, J_{4}, J\right) \lambda_{\varphi}^{\left(J_{1}\right)} \lambda_{\varphi}^{\left(J_{2}\right)} \lambda_{\varphi}^{\left(J_{4}\right)} \\
& +\frac{\lambda_{\varphi}^{(J)}\left(\lambda_{\varphi H}^{\prime}\right)^{2}}{32}\left(\mathcal{J}(J)-\mathcal{J}\left(j_{\varphi}\right)\right) \\
& +\frac{\left(\lambda_{\varphi H}^{\prime}\right)^{2}}{32 \mathcal{D}\left(j_{\varphi}\right)} \sum_{J_{1}}^{\prime} \lambda_{\varphi}^{\left(J_{1}\right)}\left(\frac{\mathcal{J}(J) \mathcal{J}\left(J_{1}\right) \mathcal{D}\left(J_{1}\right)}{\mathcal{J}\left(j_{\varphi}\right)}\right. \\
& \left.-2\left(\mathcal{J}\left(J_{1}\right)+\mathcal{J}(J)\right) \mathcal{D}\left(J_{1}\right)+4 \mathcal{D}\left(J_{1}\right) \mathcal{J}\left(j_{\varphi}\right)\right) \\
& +\frac{\left(\lambda_{\varphi H}^{\prime}\right)^{2}}{64}\left(\lambda_{H}+6 y_{t}^{2}+6 y_{t}^{2}+6 y_{t}^{2}+2 y_{\tau}^{2}\right)\left(\mathcal{J}(J)-2 \mathcal{J}\left(j_{\varphi}\right)\right) \\
& +\frac{\lambda_{\varphi H}\left(\lambda_{\varphi H}^{\prime}\right)^{2}}{32}\left(\mathcal{J}(J)-\mathcal{J}\left(j_{\varphi}\right)\right)+\frac{3}{8} \lambda_{\varphi H} \lambda_{H}+\frac{\lambda_{\varphi H}^{3}}{8} \\
& +\frac{\lambda_{\varphi H}^{2}}{4}\left(\frac{3 \lambda_{\varphi}^{(J)}}{2}+3 y_{t}^{2}+3 y_{b}^{2}+3 y_{c}^{2}+y_{\tau}^{2}\right)+\frac{\lambda_{\varphi H}^{2}}{2} \sum_{J_{1}}^{\prime} \lambda_{\varphi}^{(J)} \frac{\mathcal{D}(J)}{\mathcal{D}\left(j_{\varphi}\right)} .
\end{aligned}
$$

For the Higgs-portal couplings we find

$$
\begin{aligned}
& \Lambda_{\varphi H, 60}^{(2)}=Y_{\varphi}^{4}\left(\frac{1}{8} \mathcal{D}\left(j_{\varphi}\right)-\frac{9}{16}\right)-Y_{\varphi}^{2}\left(\frac{5}{16}-\frac{10}{3} n_{g}\right), \\
& \Lambda_{\varphi H, 42}^{(2)}=-Y_{\varphi}^{2}\left(\frac{9}{4} \mathcal{J}\left(j_{\varphi}\right)+\frac{27}{16}\right), \\
& \Lambda_{\varphi H, 24}^{(2)}=-\frac{9}{4} \mathcal{J}\left(j_{\varphi}\right)\left(1+Y_{\varphi}^{2}\right), \\
& \Lambda_{\varphi H, 06}^{(2)}=-\mathcal{J}\left(j_{\varphi}\right)\left(\frac{199}{4}-8 n_{g}+9 \mathcal{J}\left(j_{\varphi}\right)-\frac{2}{3} \mathcal{J}\left(j_{\varphi}\right) \mathcal{D}\left(j_{\varphi}\right)\right),
\end{aligned}
$$




$$
\begin{aligned}
& \Lambda_{\varphi H, 40}^{(2)}=Y_{\varphi}^{2}\left(\frac{9}{8} \lambda_{H}+\frac{1}{4}\left(9 y_{t}^{2}+9 y_{b}^{2}+9 y_{c}^{2}+3 y_{\tau}^{2}\right)+\frac{3}{4} \sum_{J_{1}}^{\prime} \lambda_{\varphi}^{\left(J_{1}\right)} \frac{\mathcal{D}\left(J_{1}\right)}{\mathcal{D}\left(j_{\varphi}\right)}\right) \\
& +Y_{\varphi}^{2} \lambda_{\varphi H}\left(\frac{5}{4}-\frac{\mathcal{D}\left(j_{\varphi}\right)}{32}\right)+\frac{Y_{\varphi}^{4}}{32} \lambda_{\varphi H}\left(15+5 \mathcal{D}\left(j_{\varphi}\right)\right)+\lambda_{\varphi H}\left[\frac{25}{32}-\frac{5}{6} n_{g}\left(1+Y_{\varphi}^{2}\right)\right] \text {, } \\
& \Lambda_{\varphi H, 22}^{(2)}=\frac{3 \mathcal{J}\left(j_{\varphi}\right)}{2} \lambda_{\varphi H}^{\prime} Y_{\varphi}+\lambda_{\varphi H}\left(\frac{45}{16}+\frac{27}{16} Y_{\varphi}^{2}+\frac{9}{4} \mathcal{J}\left(j_{\varphi}\right)+\frac{15}{4} \mathcal{J}\left(j_{\varphi}\right) Y_{\varphi}^{2}\right), \\
& \Lambda_{\varphi H, 04}^{(2)}=\mathcal{J}\left(j_{\varphi}\right)\left(\frac{9}{2} \lambda_{H}+3\left(3 y_{t}^{2}+3 y_{b}^{2}+3 y_{t}^{2}+y_{\tau}^{2}\right)+3 \sum_{J_{1}}^{\prime} \lambda_{\varphi}^{\left(J_{1}\right)} \frac{\mathcal{D}\left(J_{1}\right)}{\mathcal{D}\left(j_{\varphi}\right)}\right) \\
& +\lambda_{\varphi H}\left[\frac{393}{32}-\frac{3}{2} n_{g}+\mathcal{J}\left(j_{\varphi}\right)\left(19-2 n_{g}-\frac{1}{8} \mathcal{D}\left(j_{\varphi}\right)\right)\right. \\
& \left.+\frac{15}{2} \mathcal{J}\left(j_{\varphi}\right)^{2}+\frac{5}{6} \mathcal{J}\left(j_{\varphi}\right)^{2} \mathcal{D}\left(j_{\varphi}\right)\right] \\
& \Lambda_{\varphi H, 20}^{(2)}=-\frac{9\left(\lambda_{\varphi H}^{\prime}\right)^{2}}{64} \mathcal{J}\left(j_{\varphi}\right)\left(1+Y_{\varphi}^{2}\right)-\frac{9 \lambda_{\varphi H}^{2}}{16}\left(1+Y_{\varphi}^{2}\right) \\
& -\frac{\lambda_{\varphi H}}{8}\left(35 y_{t}^{2}+23 y_{b}^{2}+35 y_{c}^{2}+21 y_{\tau}^{2}\right)-\frac{3 \lambda_{\varphi H}}{4} Y_{\varphi}^{2}\left(3 y_{t}^{2}+3 y_{b}^{2}+3 y_{c}^{2}+y_{\tau}^{2}\right) \\
& -\frac{9}{8} \lambda_{\varphi H} \lambda_{H}\left(2+Y_{\varphi}^{2}\right)-\frac{3}{4}\left(1+2 Y_{\varphi}^{2}\right) \lambda_{\varphi H} \sum_{J_{1}}^{\prime} \lambda_{\varphi}^{\left(J_{1}\right)} \frac{\mathcal{D}\left(J_{1}\right)}{\mathcal{D}\left(j_{\varphi}\right)}, \\
& \Lambda_{\varphi H, 02}^{(2)}=-\frac{\left(\lambda_{\varphi H}^{\prime}\right)^{2}}{64} \mathcal{J}\left(j_{\varphi}\right)\left(27+36 \mathcal{J}\left(j_{\varphi}\right)\right)-\frac{\lambda_{\varphi H}^{2}}{16}\left(27+36 \mathcal{J}\left(j_{\varphi}\right)\right) \\
& -\lambda_{\varphi H}\left(9 y_{t}^{2}+9 y_{b}^{2}+9 y_{c}^{2}+3 y_{\tau}^{2}\right)\left(\frac{9}{8}+\mathcal{J}\left(j_{\varphi}\right)\right) \\
& -\frac{1}{4} \lambda_{\varphi H} \lambda_{H}\left(27+18 \mathcal{J}\left(j_{\varphi}\right)\right)-\left(\frac{9}{4}+6 \mathcal{J}\left(j_{\varphi}\right)\right) \lambda_{\varphi H} \sum_{J_{1}}^{\prime} \lambda_{\varphi}^{\left(J_{1}\right)} \frac{\mathcal{D}\left(J_{1}\right)}{\mathcal{D}\left(j_{\varphi}\right)}, \\
& \Lambda_{\varphi H, 00}^{(2)}=\left(\frac{3\left(\lambda_{\varphi H}^{\prime}\right)^{2}}{16} \mathcal{J}\left(j_{\varphi}\right)+3 \lambda_{\varphi H} \lambda_{H}+\frac{3 \lambda_{\varphi H}^{2}}{4}+\lambda_{\varphi H} \sum_{J_{1}}^{\prime} \lambda_{\varphi}^{\left(J_{1}\right)} \frac{\mathcal{D}\left(J_{1}\right)}{\mathcal{D}\left(j_{\varphi}\right)}\right)\left(3 y_{t}^{2}+3 y_{b}^{2}+3 y_{c}^{2}+y_{\tau}^{2}\right) \\
& -12 \lambda_{\varphi H} g_{s}^{2}\left(y_{t}^{2}+y_{b}^{2}+y_{c}^{2}\right)+\frac{\lambda_{\varphi H}}{4}\left(9 y_{t}^{4}+9 y_{b}^{4}+9 y_{c}^{4}-5 y_{\tau}^{4}\right) \\
& +6 \lambda_{\varphi H} y_{\tau}^{2}\left(y_{t}^{2}+y_{b}^{2}+y_{c}^{2}\right)+\frac{\lambda_{\varphi H}}{2}\left(27 y_{t}^{2} y_{b}^{2}+36 y_{t}^{2} y_{c}^{2}+36 y_{b}^{2} y_{c}^{2}\right) \\
& +\frac{5 \mathcal{J}\left(j_{\varphi}\right)}{32}\left(\lambda_{\varphi H}^{\prime}\right)^{2} \lambda_{H}+\frac{27}{8} \lambda_{\varphi H} \lambda_{H}^{2}+\lambda_{\varphi H}\left(\lambda_{\varphi H}^{\prime}\right)^{2}\left(\frac{13}{64}+\frac{\mathcal{D}\left(j_{\varphi}\right)}{128}\right) \mathcal{J}\left(j_{\varphi}\right) \\
& +\frac{9}{8} \lambda_{\varphi H}^{2} \lambda_{H}+5 \lambda_{\varphi H}^{3}\left(\frac{1}{16}+\frac{\mathcal{D}\left(j_{\varphi}\right)}{32}\right) \\
& +\lambda_{\varphi H}\left(\frac{3}{4} \sum_{J_{1}}^{\prime}\left(\lambda_{\varphi}^{\left(J_{1}\right)}\right)^{2} \frac{\mathcal{D}\left(J_{1}\right)}{\mathcal{D}\left(j_{\varphi}\right)}+\sum_{J_{1}, J_{2}}^{\prime} \lambda_{\varphi}^{\left(J_{1}\right)} \lambda_{\varphi}^{\left(J_{2}\right)} \frac{\mathcal{D}\left(J_{1}\right) \mathcal{D}\left(J_{2}\right)}{\mathcal{D}\left(j_{\varphi}\right)^{2}}\right) \\
& +\frac{\left(\lambda_{\varphi H}^{\prime}\right)^{2}}{16} \sum_{J_{1}}^{\prime} \lambda_{\varphi}^{\left(J_{1}\right)} \frac{\left(\mathcal{J}\left(J_{1}\right)-\mathcal{J}\left(j_{\varphi}\right)\right) \mathcal{D}\left(J_{1}\right)}{\mathcal{D}\left(j_{\varphi}\right)} \\
& +\left(\frac{3}{2} \lambda_{\varphi H} \lambda_{H}+\frac{3}{4} \lambda_{\varphi H}^{2}\right) \sum_{J_{1}}^{\prime} \lambda_{\varphi}^{\left(J_{1}\right)} \frac{\mathcal{D}\left(J_{1}\right)}{\mathcal{D}\left(j_{\varphi}\right)}
\end{aligned}
$$


$\Lambda_{\varphi H, 60}^{\prime(2)}=0$,

$\Lambda_{\varphi H, 42}^{\prime(2)}=Y_{\varphi}^{3}\left(\frac{1}{2} \mathcal{D}\left(j_{\varphi}\right)-\frac{9}{2}\right)-Y_{\varphi}\left(\frac{7}{2}-\frac{40}{3} n_{g}\right)$,

$\Lambda_{\varphi H, 24}^{\prime(2)}=-Y_{\varphi}\left(\frac{113}{2}-8 n_{g}+18 \mathcal{J}\left(j_{\varphi}\right)-\frac{2}{3} \mathcal{D}\left(j_{\varphi}\right) \mathcal{J}\left(j_{\varphi}\right)\right)$,

$\Lambda_{\varphi H, 60}^{\prime(2)}=0$,

$\Lambda_{\varphi H, 40}^{\prime(2)}=-\frac{1}{32} Y_{\varphi}^{2}\left(1+Y_{\varphi}^{2}\right) \lambda_{\varphi H}^{\prime} \mathcal{D}\left(j_{\varphi}\right)+\frac{15}{32} Y_{\varphi}^{4}+\lambda_{\varphi H}^{\prime}\left[\frac{13}{32}+\frac{5}{4} Y_{\varphi}^{2}-\frac{5}{6} n_{g}\left(1+Y_{\varphi}^{2}\right)\right]$,

$\Lambda_{\varphi H, 22}^{\prime(2)}=\lambda_{\varphi H}^{\prime}\left[\frac{33}{16}+\frac{9}{4} \mathcal{J}\left(j_{\varphi}\right)+Y_{\varphi}^{2}\left(\frac{1}{2} \mathcal{J}\left(j_{\varphi}\right) \mathcal{D}\left(j_{\varphi}\right)+\frac{15}{4} \mathcal{J}\left(j_{\varphi}\right)+\frac{3}{16}\right)\right]+3 Y_{\varphi}\left(\lambda_{H}+2 \lambda_{\varphi H}\right)$

$+Y_{\varphi}\left(18 y_{t}^{2}+18 y_{b}^{2}+18 y_{c}^{2}+6 y_{\tau}^{2}\right)+3 Y_{\varphi} \sum_{J_{1}}^{\prime} \lambda_{\varphi}^{\left(J_{1}\right)} \frac{\left(\mathcal{J}\left(J_{1}\right)-2 \mathcal{J}\left(j_{\varphi}\right)\right) \mathcal{D}\left(J_{1}\right)}{\mathcal{J}\left(j_{\varphi}\right) \mathcal{D}\left(j_{\varphi}\right)}$

$\Lambda_{\varphi H, 04}^{\prime(2)}=\lambda_{\varphi H}^{\prime}\left[\frac{501}{32}-\frac{3}{2} n_{g}\right.$

$$
\left.+\mathcal{J}\left(j_{\varphi}\right)\left(13-\frac{1}{8} \mathcal{D}\left(j_{\varphi}\right)-2 n_{g}+\mathcal{J}\left(j_{\varphi}\right)\left(\frac{15}{2}-\frac{1}{6} \mathcal{D}\left(j_{\varphi}\right)\right)\right)\right]
$$

$\Lambda_{\varphi H, 20}^{\prime(2)}=-\frac{\lambda_{\varphi H}^{\prime}}{8}\left(35 y_{t}^{2}+23 y_{b}^{2}+35 y_{c}^{2}+21 y_{\tau}^{2}\right)-\frac{3 \lambda_{\varphi H}^{\prime}}{4} Y_{\varphi}^{2}\left(3 y_{t}^{2}+3 y_{b}^{2}+3 y_{c}^{2}+y_{\tau}^{2}\right)$

$$
\begin{aligned}
& -\frac{3}{8} \lambda_{\varphi H}^{\prime} \lambda_{H}\left(2+Y_{\varphi}^{2}\right)-\frac{9}{8} \lambda_{\varphi H}^{\prime} \lambda_{\varphi H}\left(1+Y_{\varphi}^{2}\right) \\
& -\frac{3}{8}\left(1+2 Y_{\varphi}^{2}\right) \lambda_{\varphi H}^{\prime} \sum_{J_{1}}^{\prime} \lambda_{\varphi}^{\left(J_{1}\right)} \frac{\left(\mathcal{J}\left(J_{1}\right)-2 \mathcal{J}\left(j_{\varphi}\right)\right) \mathcal{D}\left(J_{1}\right)}{\mathcal{J}\left(j_{\varphi}\right) \mathcal{D}\left(j_{\varphi}\right)},
\end{aligned}
$$

$\Lambda_{\varphi H, 02}^{\prime(2)}=-\frac{\lambda_{\varphi H}^{\prime} \lambda_{\varphi H}}{8} \mathcal{J}\left(j_{\varphi}\right)\left(27+36 \mathcal{J}\left(j_{\varphi}\right)\right)-\frac{\lambda_{\varphi H}^{\prime} \lambda_{H}}{4}\left(9+6 \mathcal{J}\left(j_{\varphi}\right)\right)$

$$
\begin{aligned}
& -\lambda_{\varphi H}^{\prime}\left(9 y_{t}^{2}+9 y_{b}^{2}+9 y_{c}^{2}+3 y_{\tau}^{2}\right)\left(\frac{9}{8}+\mathcal{J}\left(j_{\varphi}\right)\right) \\
& -\lambda_{\varphi H}^{\prime}\left(\frac{9}{8}+3 \mathcal{J}\left(j_{\varphi}\right)\right) \sum_{J_{1}}^{\prime} \lambda_{\varphi}^{\left(J_{1}\right)} \frac{\left(\mathcal{J}\left(J_{1}\right)-2 \mathcal{J}\left(j_{\varphi}\right)\right) \mathcal{D}\left(J_{1}\right)}{\mathcal{J}\left(j_{\varphi}\right) \mathcal{D}\left(j_{\varphi}\right)}
\end{aligned}
$$

$\Lambda_{\varphi H, 00}^{\prime(2)}=\left[\lambda_{\varphi H}^{\prime} \lambda_{H}+\frac{3 \lambda_{\varphi H} \lambda_{\varphi H}^{\prime}}{2}+\frac{1}{2} \sum_{J_{1}}^{\prime} \lambda_{\varphi}^{\left(J_{1}\right)}\left(\frac{\mathcal{J}\left(J_{1}\right) \mathcal{D}\left(J_{1}\right)}{\mathcal{J}\left(j_{\varphi}\right) \mathcal{D}\left(j_{\varphi}\right)}-2 \frac{\mathcal{D}\left(J_{1}\right)}{\mathcal{D}\left(j_{\varphi}\right)}\right)\right]$

$$
\times\left(3 y_{t}^{2}+3 y_{b}^{2}+3 y_{c}^{2}+y_{\tau}^{2}\right)
$$$$
-12 \lambda_{\varphi H}^{\prime} g_{s}^{2}\left(y_{t}^{2}+y_{b}^{2}+y_{c}^{2}\right)+\frac{3 \lambda_{\varphi H}^{\prime}}{4}\left(11 y_{t}^{4}+11 y_{b}^{4}+11 y_{c}^{4}+y_{\tau}^{4}\right)
$$$$
+6 \lambda_{\varphi H}^{\prime} y_{\tau}^{2}\left(y_{t}^{2}+y_{b}^{2}+y_{c}^{2}\right)+\frac{\lambda_{\varphi H}^{\prime}}{2}\left(27 y_{t}^{2} y_{b}^{2}+36 y_{t}^{2} y_{c}^{2}+36 y_{b}^{2} y_{c}^{2}\right)
$$$$
+\frac{5}{4} \lambda_{\varphi H} \lambda_{\varphi H}^{\prime} \lambda_{H}+\frac{7}{8} \lambda_{\varphi H}^{\prime} \lambda_{H}^{2}+\lambda_{\varphi H}^{\prime} \lambda_{\varphi H}^{2}\left(\frac{13}{16}+\frac{\mathcal{D}\left(j_{\varphi}\right)}{32}\right)
$$

$+\left(\lambda_{\varphi H}\right)^{3}\left(\frac{\mathcal{J}\left(j_{\varphi}\right) \mathcal{D}\left(j_{\varphi}\right)}{128}+\frac{5 \mathcal{J}\left(j_{\varphi}\right)}{64}-\frac{1}{64}\right)$ 


$$
\begin{aligned}
& +\frac{\lambda_{\varphi H}^{\prime}}{4} \sum_{J_{1}}^{\prime}\left(\lambda_{\varphi}^{\left(J_{1}\right)}\right)^{2}\left(\frac{\mathcal{J}\left(J_{1}\right) \mathcal{D}\left(J_{1}\right)}{\mathcal{J}\left(j_{\varphi}\right) \mathcal{D}\left(j_{\varphi}\right)}-\frac{\mathcal{D}\left(J_{1}\right)}{\mathcal{D}\left(j_{\varphi}\right)}\right) \\
& +\frac{\lambda_{\varphi H}^{\prime}}{4 \mathcal{D}\left(j_{\varphi}\right)^{2}} \sum_{J_{1}, J_{2}}^{\prime} \lambda_{\varphi}^{\left(J_{1}\right)} \lambda_{\varphi}^{\left(J_{2}\right)}\left(\frac{\mathcal{J}\left(J_{1}\right) \mathcal{D}\left(J_{1}\right) \mathcal{J}\left(J_{2}\right) \mathcal{D}\left(J_{2}\right)}{\mathcal{J}\left(j_{\varphi}\right)^{2}}\right. \\
& \left.\quad-2 \frac{\left(\mathcal{J}\left(J_{1}\right)+\mathcal{J}\left(J_{2}\right)\right) \mathcal{D}\left(J_{1}\right) \mathcal{D}\left(J_{2}\right)}{\mathcal{J}\left(j_{\varphi}\right)}+4 \mathcal{D}\left(J_{1}\right) \mathcal{D}\left(J_{2}\right)\right) \\
& +\frac{\lambda_{\varphi H}^{\prime} \lambda_{H}}{4} \sum_{J_{1}}^{\prime} \lambda_{\varphi}^{\left(J_{1}\right)}\left(\frac{\mathcal{J}\left(J_{1}\right) \mathcal{D}\left(J_{1}\right)}{\mathcal{J}\left(j_{\varphi}\right) \mathcal{D}\left(j_{\varphi}\right)}-2 \frac{\mathcal{D}\left(J_{1}\right)}{\mathcal{D}\left(j_{\varphi}\right)}\right) \\
& +\frac{\lambda_{\varphi H} \lambda_{\varphi H}^{\prime}}{2} \sum_{J_{1}}^{\prime} \lambda_{\varphi}^{\left(J_{1}\right)}\left(\frac{\mathcal{J}\left(J_{1}\right) \mathcal{D}\left(J_{1}\right)}{\mathcal{J}\left(j_{\varphi}\right) \mathcal{D}\left(j_{\varphi}\right)}-\frac{\mathcal{D}\left(J_{1}\right)}{\mathcal{D}\left(j_{\varphi}\right)}\right) .
\end{aligned}
$$

Our results for the quartic Higgs self coupling are

$$
\begin{aligned}
& \Lambda_{H, 60}^{(2)}=\frac{1}{16} Y_{\varphi}^{2} \mathcal{D}\left(j_{\varphi}\right)-\frac{7}{16}+\frac{5}{3} n_{g}, \\
& \Lambda_{H, 42}^{(2)}=\frac{1}{16} Y_{\varphi}^{2} \mathcal{D}\left(j_{\varphi}\right)-\frac{43}{16}+\frac{5}{3} n_{g}, \\
& \Lambda_{H, 24}^{(2)}=\frac{1}{12} \mathcal{J}\left(j_{\varphi}\right) \mathcal{D}\left(j_{\varphi}\right)-\frac{167}{16}+n_{g}, \\
& \Lambda_{H, 06}^{(2)}=\frac{1}{4} \mathcal{J}\left(j_{\varphi}\right) \mathcal{D}\left(j_{\varphi}\right)-\frac{339}{16}+3 n_{g}, \\
& \Lambda_{H, 40}^{(2)}=\frac{3}{16} \lambda_{\varphi H} Y_{\varphi}^{2} \mathcal{D}\left(j_{\varphi}\right)+\frac{13}{4} \lambda_{H}-\frac{1}{16} \lambda_{H} Y_{\varphi}^{2} \mathcal{D}\left(j_{\varphi}\right)-\lambda_{H} \frac{5}{3} n_{g} \\
& +\frac{3}{4}\left(3 y_{t}^{2}+3 y_{b}^{2}+3 y_{c}^{2}+y_{\tau}^{2}\right) \text {, } \\
& \Lambda_{H, 22}^{(2)}=\frac{45}{4} \lambda_{H}+\frac{\lambda_{\varphi H}^{\prime}}{8} Y_{\varphi} \mathcal{J}\left(j_{\varphi}\right) \mathcal{D}\left(j_{\varphi}\right)+\frac{3}{2}\left(3 y_{t}^{2}+3 y_{b}^{2}+3 y_{c}^{2}+y_{\tau}^{2}\right) \text {, } \\
& \Lambda_{H, 04}^{(2)}=\frac{3}{4} \lambda_{\varphi H} \mathcal{J}\left(j_{\varphi}\right) \mathcal{D}\left(j_{\varphi}\right)+\frac{9}{4}\left(3 y_{t}^{2}+3 y_{b}^{2}+3 y_{c}^{2}+y_{\tau}^{2}\right) \\
& -\lambda_{H}\left(3 n_{g}-33+\frac{1}{4} \mathcal{J}\left(j_{\varphi}\right) \mathcal{D}\left(j_{\varphi}\right)\right) \text {, } \\
& \Lambda_{H, 20}^{(2)}=-\frac{27}{4} \lambda_{H}^{2}-\frac{3}{32} \lambda_{\varphi H}^{2}\left(2+Y_{\varphi}^{2}\right) \mathcal{D}\left(j_{\varphi}\right)-\frac{1}{128}\left(\lambda_{\varphi H}^{\prime}\right)^{2}\left(2+Y_{\varphi}^{2}\right) \mathcal{J}\left(j_{\varphi}\right) \mathcal{D}\left(j_{\varphi}\right) \\
& -\frac{\lambda_{H}}{4}\left(53 y_{t}^{2}+41 y_{b}^{2}+53 y_{c}^{2}+27 y_{\tau}^{2}\right)+26 y_{t}^{4}+14 y_{b}^{4}+26 y_{c}^{4}+18 y_{\tau}^{4} \text {, } \\
& \Lambda_{H, 02}^{(2)}=-\frac{81}{4} \lambda_{H}^{2}-\frac{1}{16} \lambda_{\varphi H}^{2}\left(9+6 \mathcal{J}\left(j_{\varphi}\right)\right) \mathcal{D}\left(j_{\varphi}\right)-\frac{1}{64}\left(\lambda_{\varphi H}^{\prime}\right)^{2}\left(3+2 \mathcal{J}\left(j_{\varphi}\right)\right) \mathcal{J}\left(j_{\varphi}\right) \mathcal{D}\left(j_{\varphi}\right) \\
& -\frac{45}{4} \lambda_{H}\left(3 y_{t}^{2}+3 y_{b}^{2}+3 y_{c}^{2}+y_{\tau}^{2}\right)+54 y_{t}^{4}+54 y_{b}^{4}+54 y_{c}^{4}+18 y_{\tau}^{4} \text {, } \\
& \Lambda_{H, 00}^{(2)}=9 \lambda_{H}^{3}+\frac{\mathcal{D}\left(j_{\varphi}\right)}{16} \lambda_{\varphi H}^{3}+\frac{9}{16} \lambda_{\varphi H}^{2} \lambda_{H} \mathcal{D}\left(j_{\varphi}\right)+\frac{7}{192}\left(\lambda_{\varphi H}^{\prime}\right)^{2} \lambda_{H} \mathcal{J}\left(j_{\varphi}\right) \mathcal{D}\left(j_{\varphi}\right) \\
& +\frac{5}{192}\left(\lambda_{\varphi H}^{\prime}\right)^{2} \lambda_{\varphi H} \mathcal{J}\left(j_{\varphi}\right) \mathcal{D}\left(j_{\varphi}\right)+\frac{\lambda_{\varphi H}^{2}}{8} \sum_{J_{1}}^{\prime} \lambda_{\varphi}^{\left(J_{1}\right)} \mathcal{D}\left(J_{1}\right) \\
& +\frac{\left(\lambda_{\varphi H}^{\prime}\right)^{2}}{192} \sum_{J_{1}}^{\prime} \lambda_{\varphi}^{\left(J_{1}\right)} \mathcal{D}\left(J_{1}\right)\left(\mathcal{J}\left(J_{1}\right)-2 \mathcal{J}\left(j_{\varphi}\right)\right)
\end{aligned}
$$




$$
\begin{aligned}
& +\left(9 \lambda_{H}^{2}+\frac{\mathcal{D}\left(j_{\varphi}\right)}{4} \lambda_{\varphi H}^{2}+\frac{\mathcal{J}\left(j_{\varphi}\right) \mathcal{D}\left(j_{\varphi}\right)}{48}\left(\lambda_{\varphi H}^{\prime}\right)^{2}\right)\left(3 y_{t}^{2}+3 y_{b}^{2}+3 y_{c}^{2}+y_{\tau}^{2}\right) \\
& -\frac{\lambda_{H}}{2}\left(9 y_{t}^{4}+9 y_{b}^{4}+9 y_{c}^{4}+15 y_{\tau}^{4}-90 y_{t}^{2} y_{b}^{2}-108 y_{t}^{2} y_{c}^{2}-108 y_{b}^{2} y_{c}^{2}\right) \\
& +18 \lambda_{H}\left(y_{t}^{2}+y_{b}^{2}+y_{c}^{2}\right) y_{\tau}^{2}-24 \lambda_{H} g_{s}^{2}\left(y_{t}^{2}+y_{b}^{2}+y_{c}^{2}\right) \\
& -90\left(y_{t}^{6}+y_{b}^{6}+y_{c}^{6}\right)-14 y_{\tau}^{6}-24\left(y_{t}^{2}+y_{b}^{2}+y_{c}^{2}\right) y_{\tau}^{4}-24\left(y_{t}^{4}+y_{b}^{4}+y_{c}^{4}\right) y_{\tau}^{2} \\
& +96 g_{s}^{2}\left(y_{t}^{4}+y_{b}^{4}+y_{c}^{4}\right)-54\left(y_{t}^{4} y_{b}^{2}+y_{t}^{2} y_{b}^{4}\right)-72\left(y_{t}^{4} y_{c}^{2}+y_{t}^{2} y_{c}^{4}\right) \\
& -72\left(y_{b}^{4} y_{c}^{2}+y_{b}^{2} y_{c}^{4}\right) .
\end{aligned}
$$

The full two-loop contributions to the fermion, scalar, and Higgs field renormalization constants are

$$
\begin{aligned}
& \delta Z_{t_{R}}^{(2)}=\frac{g_{1}^{4}}{\left(16 \pi^{2}\right)^{2} \epsilon}\left(\frac{11}{54}+\frac{20}{27} n_{g}+\frac{1}{36} \mathcal{D}\left(j_{\varphi}\right) Y_{\varphi}^{2}\right)+\frac{8}{81} \frac{g_{1}^{4}}{\left(16 \pi^{2}\right)^{2} \epsilon^{2}} \xi_{B}^{2} \\
& -\frac{g_{s}^{4}}{\left(16 \pi^{2}\right)^{2} \epsilon}\left(\frac{67}{6}+4 \xi_{G}+\frac{1}{2} \xi_{G}^{2}-\frac{4}{3} n_{g}\right)+\frac{g_{s}^{4}}{\left(16 \pi^{2}\right)^{2} \epsilon^{2}}\left(3 \xi_{G}+\frac{17}{9} \xi_{G}^{2}\right) \\
& +\frac{8}{9} \frac{g_{1}^{2} g_{s}^{2}}{\left(16 \pi^{2}\right)^{2} \epsilon}+\frac{16}{27} \frac{g_{1}^{2} g_{s}^{2}}{\left(16 \pi^{2}\right)^{2} \epsilon^{2}} \xi_{B} \xi_{G}-\frac{y_{t}^{2}}{\left(16 \pi^{2}\right)^{2} \epsilon}\left(\frac{49}{144} g_{1}^{2}+\frac{51}{16} g_{2}^{2}-\frac{8}{3} g_{s}^{2}\right) \\
& +\frac{y_{t}^{2}}{\left(16 \pi^{2}\right)^{2} \epsilon^{2}}\left[\left(\frac{17}{24}+\frac{4}{9} \xi_{B}\right) g_{1}^{2}+\frac{9}{8} g_{2}^{2}+\left(4+\frac{4}{3} \xi_{G}\right) g_{s}^{2}\right] \\
& +\frac{y_{t}^{2}}{\left(16 \pi^{2}\right)^{2} \epsilon}\left(\frac{19}{8} y_{t}^{2}+\frac{19}{8} y_{b}^{2}+\frac{9}{4} y_{c}^{2}+\frac{3}{4} y_{\tau}^{2}\right) \\
& -\frac{y_{t}^{2}}{\left(16 \pi^{2}\right)^{2} \epsilon^{2}}\left(\frac{7}{4} y_{t}^{2}+\frac{3}{4} y_{b}^{2}+\frac{3}{2} y_{c}^{2}+\frac{1}{2} y_{\tau}^{2}\right) \\
& \delta Z_{b_{R}}^{(2)}=\frac{g_{1}^{4}}{\left(16 \pi^{2}\right)^{2} \epsilon}\left(\frac{5}{216}+\frac{5}{27} n_{g}+\frac{1}{144} \mathcal{D}\left(j_{\varphi}\right) Y_{\varphi}^{2}\right)+\frac{1}{162} \frac{g_{1}^{4}}{\left(16 \pi^{2}\right)^{2} \epsilon^{2}} \xi_{B}^{2} \\
& -\frac{g_{s}^{4}}{\left(16 \pi^{2}\right)^{2} \epsilon}\left(\frac{67}{6}+4 \xi_{G}+\frac{1}{2} \xi_{G}^{2}-\frac{4}{3} n_{g}\right)+\frac{g_{s}^{4}}{\left(16 \pi^{2}\right)^{2} \epsilon^{2}}\left(3 \xi_{G}+\frac{17}{9} \xi_{G}^{2}\right) \\
& +\frac{2}{9} \frac{g_{1}^{2} g_{s}^{2}}{\left(16 \pi^{2}\right)^{2} \epsilon}+\frac{4}{27} \frac{g_{1}^{2} g_{s}^{2}}{\left(16 \pi^{2}\right)^{2} \epsilon^{2}} \xi_{B} \xi_{G}-\frac{y_{b}^{2}}{\left(16 \pi^{2}\right)^{2} \epsilon}\left(\frac{133}{144} g_{1}^{2}+\frac{51}{16} g_{2}^{2}-\frac{8}{3} g_{s}^{2}\right) \\
& +\frac{y_{b}^{2}}{\left(16 \pi^{2}\right)^{2} \epsilon^{2}}\left[\left(\frac{5}{24}+\frac{1}{9} \xi_{B}\right) g_{1}^{2}+\frac{9}{8} g_{2}^{2}+\left(4+\frac{4}{3} \xi_{G}\right) g_{s}^{2}\right] \\
& +\frac{y_{b}^{2}}{\left(16 \pi^{2}\right)^{2} \epsilon}\left(\frac{19}{8} y_{t}^{2}+\frac{19}{8} y_{b}^{2}+\frac{9}{4} y_{c}^{2}+\frac{3}{4} y_{\tau}^{2}\right) \\
& -\frac{y_{t}^{2}}{\left(16 \pi^{2}\right)^{2} \epsilon^{2}}\left(\frac{3}{4} y_{t}^{2}+\frac{7}{4} y_{b}^{2}+\frac{3}{2} y_{c}^{2}+\frac{1}{2} y_{\tau}^{2}\right) \\
& \delta Z_{c_{R}}^{(2)}=\frac{g_{1}^{4}}{\left(16 \pi^{2}\right)^{2} \epsilon}\left(\frac{11}{54}+\frac{20}{27} n_{g}+\frac{1}{36} \mathcal{D}\left(j_{\varphi}\right) Y_{\varphi}^{2}\right)+\frac{8}{81} \frac{g_{1}^{4}}{\left(16 \pi^{2}\right)^{2} \epsilon^{2}} \xi_{B}^{2} \\
& -\frac{g_{s}^{4}}{\left(16 \pi^{2}\right)^{2} \epsilon}\left(\frac{67}{6}+4 \xi_{G}+\frac{1}{2} \xi_{G}^{2}-\frac{4}{3} n_{g}\right)+\frac{g_{s}^{4}}{\left(16 \pi^{2}\right)^{2} \epsilon^{2}}\left(3 \xi_{G}+\frac{17}{9} \xi_{G}^{2}\right) \\
& +\frac{8}{9} \frac{g_{1}^{2} g_{s}^{2}}{\left(16 \pi^{2}\right)^{2} \epsilon}+\frac{16}{27} \frac{g_{1}^{2} g_{s}^{2}}{\left(16 \pi^{2}\right)^{2} \epsilon^{2}} \xi_{B} \xi_{G}-\frac{y_{c}^{2}}{\left(16 \pi^{2}\right)^{2} \epsilon}\left(\frac{49}{144} g_{1}^{2}+\frac{51}{16} g_{2}^{2}-\frac{8}{3} g_{s}^{2}\right)
\end{aligned}
$$




$$
\begin{aligned}
& +\frac{y_{c}^{2}}{\left(16 \pi^{2}\right)^{2} \epsilon^{2}}\left[\left(\frac{17}{24}+\frac{4}{9} \xi_{B}\right) g_{1}^{2}+\frac{9}{8} g_{2}^{2}+\left(4+\frac{4}{3} \xi_{G}\right) g_{s}^{2}\right] \\
& +\frac{y_{c}^{2}}{\left(16 \pi^{2}\right)^{2} \epsilon}\left(\frac{9}{4} y_{t}^{2}+\frac{9}{4} y_{b}^{2}+\frac{19}{8} y_{c}^{2}+\frac{3}{4} y_{\tau}^{2}\right) \\
& -\frac{y_{c}^{2}}{\left(16 \pi^{2}\right)^{2} \epsilon^{2}}\left(\frac{3}{2} y_{t}^{2}+\frac{3}{2} y_{b}^{2}+\frac{7}{4} y_{c}^{2}+\frac{1}{2} y_{\tau}^{2}\right) \\
& \delta Z_{Q_{t, L}}^{(2)}=\frac{g_{1}^{4}}{\left(16 \pi^{2}\right)^{2} \epsilon}\left(\frac{7}{1728}+\frac{5}{108} n_{g}+\frac{1}{576} \mathcal{D}\left(j_{\varphi}\right) Y_{\varphi}^{2}\right)+\frac{1}{2592} \frac{g_{1}^{4}}{\left(16 \pi^{2}\right)^{2} \epsilon^{2}} \xi_{B}^{2} \\
& -\frac{g_{2}^{4}}{\left(16 \pi^{2}\right)^{2} \epsilon}\left(\frac{267}{64}+\frac{3}{2} \xi_{W}+\frac{3}{16} \xi_{W}^{2}-\frac{3}{4} n_{g}-\frac{1}{16} \mathcal{J}\left(j_{\varphi}\right) \mathcal{D}\left(j_{\varphi}\right)\right) \\
& +\frac{g_{2}^{4}}{\left(16 \pi^{2}\right)^{2} \epsilon^{2}}\left(\frac{9}{8} \xi_{W}+\frac{21}{32} \xi_{W}^{2}\right)-\frac{g_{s}^{4}}{\left(16 \pi^{2}\right)^{2} \epsilon}\left(\frac{67}{6}+4 \xi_{G}+\frac{1}{2} \xi_{G}^{2}-\frac{4}{3} n_{g}\right) \\
& +\frac{g_{s}^{4}}{\left(16 \pi^{2}\right)^{2} \epsilon^{2}}\left(3 \xi_{G}+\frac{17}{9} \xi_{G}^{2}\right)+\frac{1}{32} \frac{g_{1}^{2} g_{2}^{2}}{\left(16 \pi^{2}\right)^{2} \epsilon}+\frac{1}{48} \frac{g_{1}^{2} g_{2}^{2}}{\left(16 \pi^{2}\right)^{2} \epsilon^{2}} \xi_{B} \xi_{W} \\
& +\frac{1}{18} \frac{g_{1}^{2} g_{s}^{2}}{\left(16 \pi^{2}\right)^{2} \epsilon}+\frac{1}{27} \frac{g_{1}^{2} g_{s}^{2}}{\left(16 \pi^{2}\right)^{2} \epsilon^{2}} \xi_{B} \xi_{G}+\frac{3}{2} \frac{g_{2}^{2} g_{s}^{2}}{\left(16 \pi^{2}\right)^{2} \epsilon}+\frac{g_{2}^{2} g_{s}^{2}}{\left(16 \pi^{2}\right)^{2} \epsilon^{2}} \xi_{W} \xi_{G} \\
& -\frac{1}{\left(16 \pi^{2}\right)^{2} \epsilon}\left[\left(\frac{139}{288} y_{t}^{2}+\frac{151}{288} y_{b}^{2}\right) g_{1}^{2}+\frac{33}{32}\left(y_{t}^{2}+y_{b}^{2}\right) g_{2}^{2}-\frac{4}{3}\left(y_{t}^{2}+y_{b}^{2}\right) g_{s}^{2}\right] \\
& +\frac{1}{\left(16 \pi^{2}\right)^{2} \epsilon^{2}}\left[\left(\frac{17}{48} y_{t}^{2}+\frac{5}{48} y_{b}^{2}+\frac{1}{72}\left(y_{t}^{2}+y_{b}^{2}\right) \xi_{B}\right) g_{1}^{2}\right. \\
& \left.+\left(\frac{9}{16}+\frac{3}{8} \xi_{W}\right)\left(y_{t}^{2}+y_{b}^{2}\right) g_{2}^{2}+\left(2+\frac{2}{3} \xi_{G}\right)\left(y_{t}^{2}+y_{b}^{2}\right) g_{s}^{2}\right] \\
& +\frac{y_{t}^{2}}{\left(16 \pi^{2}\right)^{2} \epsilon}\left(\frac{5}{4} y_{t}^{2}+\frac{9}{8} y_{b}^{2}+\frac{9}{8} y_{c}^{2}+\frac{3}{8} y_{\tau}^{2}\right)-\frac{y_{t}^{2}}{\left(16 \pi^{2}\right)^{2} \epsilon^{2}}\left(y_{t}^{2}+\frac{1}{4} y_{t}^{2}+\frac{3}{4} y_{c}^{2}+\frac{1}{4} y_{\tau}^{2}\right) \\
& +\frac{y_{b}^{2}}{\left(16 \pi^{2}\right)^{2} \epsilon}\left(\frac{9}{8} y_{b}^{2}+\frac{5}{4} y_{b}^{2}+\frac{9}{8} y_{c}^{2}+\frac{3}{8} y_{\tau}^{2}\right)-\frac{y_{b}^{2}}{\left(16 \pi^{2}\right)^{2} \epsilon^{2}}\left(\frac{1}{4} y_{t}^{2}+y_{b}^{2}+\frac{3}{4} y_{c}^{2}+\frac{1}{4} y_{\tau}^{2}\right) \\
& \delta Z_{Q_{c, L}}^{(2)}=\frac{g_{1}^{4}}{\left(16 \pi^{2}\right)^{2} \epsilon}\left(\frac{7}{1728}+\frac{5}{108} n_{g}+\frac{1}{576} \mathcal{D}\left(j_{\varphi}\right) Y_{\varphi}^{2}\right)+\frac{1}{2592} \frac{g_{1}^{4}}{\left(16 \pi^{2}\right)^{2} \epsilon^{2}} \xi_{B}^{2} \\
& -\frac{g_{2}^{4}}{\left(16 \pi^{2}\right)^{2} \epsilon}\left(\frac{267}{64}+\frac{3}{2} \xi_{W}+\frac{3}{16} \xi_{W}^{2}-\frac{3}{4} n_{g}-\frac{1}{16} \mathcal{J}\left(j_{\varphi}\right) \mathcal{D}\left(j_{\varphi}\right)\right) \\
& +\frac{g_{2}^{4}}{\left(16 \pi^{2}\right)^{2} \epsilon^{2}}\left(\frac{9}{8} \xi_{W}+\frac{21}{32} \xi_{W}^{2}\right)-\frac{g_{s}^{4}}{\left(16 \pi^{2}\right)^{2} \epsilon}\left(\frac{67}{6}+4 \xi_{G}+\frac{1}{2} \xi_{G}^{2}-\frac{4}{3} n_{g}\right) \\
& +\frac{g_{s}^{4}}{\left(16 \pi^{2}\right)^{2} \epsilon^{2}}\left(3 \xi_{G}+\frac{17}{9} \xi_{G}^{2}\right)+\frac{1}{32} \frac{g_{1}^{2} g_{2}^{2}}{\left(16 \pi^{2}\right)^{2} \epsilon}+\frac{1}{48} \frac{g_{1}^{2} g_{2}^{2}}{\left(16 \pi^{2}\right)^{2} \epsilon^{2}} \xi_{B} \xi_{W} \\
& +\frac{1}{18} \frac{g_{1}^{2} g_{s}^{2}}{\left(16 \pi^{2}\right)^{2} \epsilon}+\frac{1}{27} \frac{g_{1}^{2} g_{s}^{2}}{\left(16 \pi^{2}\right)^{2} \epsilon^{2}} \xi_{B} \xi_{G}+\frac{3}{2} \frac{g_{2}^{2} g_{s}^{2}}{\left(16 \pi^{2}\right)^{2} \epsilon}+\frac{g_{2}^{2} g_{s}^{2}}{\left(16 \pi^{2}\right)^{2} \epsilon^{2}} \xi_{W} \xi_{G} \\
& -\frac{y_{c}^{2}}{\left(16 \pi^{2}\right)^{2} \epsilon}\left(\frac{139}{288} g_{1}^{2}+\frac{33}{32} g_{2}^{2}-\frac{4}{3} g_{s}^{2}\right) \\
& +\frac{y_{c}^{2}}{\left(16 \pi^{2}\right)^{2} \epsilon^{2}}\left[\left(\frac{17}{48}+\frac{1}{72} \xi_{B}\right) g_{1}^{2}+\left(\frac{9}{16}+\frac{3}{8} \xi_{W}\right) g_{2}^{2}+\left(2+\frac{2}{3} \xi_{G}\right) g_{s}^{2}\right] \\
& +\frac{y_{c}^{2}}{\left(16 \pi^{2}\right)^{2} \epsilon}\left(\frac{9}{8} y_{t}^{2}+\frac{9}{8} y_{b}^{2}+\frac{5}{4} y_{c}^{2}+\frac{3}{8} y_{\tau}^{2}\right)-\frac{y_{c}^{2}}{\left(16 \pi^{2}\right)^{2} \epsilon^{2}}\left(\frac{3}{4} y_{t}^{2}+\frac{3}{4} y_{b}^{2}+y_{c}^{2}+\frac{1}{4} y_{\tau}^{2}\right)
\end{aligned}
$$




$$
\begin{aligned}
& \delta Z_{\tau_{R}}^{(2)}=\frac{g_{1}^{4}}{\left(16 \pi^{2}\right)^{2} \epsilon}\left(\frac{7}{8}+\frac{5}{3} n_{g}+\frac{1}{16} \mathcal{D}\left(j_{\varphi}\right) Y_{\varphi}^{2}\right)+\frac{1}{2} \frac{g_{1}^{4}}{\left(16 \pi^{2}\right)^{2} \epsilon^{2}} \xi_{B}^{2} \\
& +\frac{11}{16} \frac{y_{\tau}^{2} g_{1}^{2}}{\left(16 \pi^{2}\right)^{2} \epsilon}+\frac{y_{\tau}^{2} g_{1}^{2}}{\left(16 \pi^{2}\right)^{2} \epsilon^{2}}\left(\frac{15}{8}+\xi_{B}\right)-\frac{51}{16} \frac{y_{\tau}^{2} g_{2}^{2}}{\left(16 \pi^{2}\right)^{2} \epsilon}+\frac{9}{8} \frac{y_{\tau}^{2} g_{1}^{2}}{\left(16 \pi^{2}\right)^{2} \epsilon^{2}} \\
& +\frac{y_{\tau}^{2}}{\left(16 \pi^{2}\right)^{2} \epsilon}\left(\frac{9}{4} y_{t}^{2}+\frac{9}{4} y_{b}^{2}+\frac{9}{4} y_{c}^{2}+\frac{7}{8} y_{\tau}^{2}\right) \\
& -\frac{y_{\tau}^{2}}{\left(16 \pi^{2}\right)^{2} \epsilon^{2}}\left(\frac{3}{2} y_{t}^{2}+\frac{3}{2} y_{b}^{2}+\frac{3}{2} y_{c}^{2}+\frac{3}{4} y_{\tau}^{2}\right) \\
& \delta Z_{L_{\tau, L}}^{(2)}=\frac{g_{1}^{4}}{\left(16 \pi^{2}\right)^{2} \epsilon}\left(\frac{5}{64}+\frac{5}{12} n_{g}+\frac{1}{64} \mathcal{D}\left(j_{\varphi}\right) Y_{\varphi}^{2}\right)+\frac{1}{32} \frac{g_{1}^{4}}{\left(16 \pi^{2}\right)^{2} \epsilon^{2}} \xi_{B}^{2} \\
& -\frac{g_{2}^{4}}{\left(16 \pi^{2}\right)^{2} \epsilon}\left(\frac{267}{64}+\frac{3}{2} \xi_{W}+\frac{3}{16} \xi_{W}^{2}-\frac{3}{4} n_{g}-\frac{1}{16} \mathcal{J}\left(j_{\varphi}\right) \mathcal{D}\left(j_{\varphi}\right)\right) \\
& +\frac{g_{2}^{4}}{\left(16 \pi^{2}\right)^{2} \epsilon^{2}}\left(\frac{9}{8} \xi_{W}+\frac{21}{32} \xi_{W}^{2}\right)+\frac{9}{32} \frac{g_{1}^{2} g_{2}^{2}}{\left(16 \pi^{2}\right)^{2} \epsilon}+\frac{3}{16} \frac{g_{1}^{2} g_{2}^{2}}{\left(16 \pi^{2}\right)^{2} \epsilon^{2}} \xi_{B} \xi_{W} \\
& -\frac{y_{\tau}^{2}}{\left(16 \pi^{2}\right)^{2} \epsilon}\left(\frac{7}{32} g_{1}^{2}+\frac{33}{32} g_{2}^{2}\right) \\
& +\frac{y_{\tau}^{2}}{\left(16 \pi^{2}\right)^{2} \epsilon^{2}}\left[\left(\frac{15}{16}+\frac{1}{8} \xi_{B}\right) g_{1}^{2}+\left(\frac{9}{16}+\frac{3}{8} \xi_{W}\right) g_{2}^{2}\right] \\
& +\frac{y_{\tau}^{2}}{\left(16 \pi^{2}\right)^{2} \epsilon}\left(\frac{9}{8} y_{t}^{2}+\frac{9}{8} y_{b}^{2}+\frac{9}{8} y_{c}^{2}+\frac{1}{2} y_{\tau}^{2}\right) \\
& -\frac{y_{\tau}^{2}}{\left(16 \pi^{2}\right)^{2} \epsilon^{2}}\left(\frac{3}{4} y_{t}^{2}+\frac{3}{4} y_{b}^{2}+\frac{3}{4} y_{c}^{2}+\frac{1}{2} y_{\tau}^{2}\right) \\
& \delta Z_{\varphi}^{(2)}=-\frac{g_{1}^{4}}{\left(16 \pi^{2}\right)^{2} \epsilon}\left[\frac{Y_{\varphi}^{4}}{64}\left(3+\frac{11}{3} \mathcal{D}\left(j_{\varphi}\right)\right)+Y_{\varphi}^{2}\left(\frac{11}{96}+\frac{25 n_{g}}{36}\right)\right] \\
& +\frac{g_{1}^{4}}{\left(16 \pi^{2}\right)^{2} \epsilon^{2}}\left[\frac{Y_{\varphi}^{4}}{32}\left(9-6 \xi_{B}+\xi_{B}^{2}+\mathcal{D}\left(j_{\varphi}\right)\right)+Y_{\varphi}^{2}\left(\frac{1}{16}+\frac{5 n_{g}}{6}\right)\right] \\
& +\frac{g_{2}^{4}}{\left(16 \pi^{2}\right)^{2} \epsilon} \mathcal{J}\left(j_{\varphi}\right)\left[\frac{269}{24}-2 \xi_{W}-\frac{\xi_{W}^{2}}{4}-\frac{5 n_{g}}{3}-\mathcal{J}\left(j_{\varphi}\right)\left(\frac{3}{4}+\frac{11}{36} \mathcal{D}\left(j_{\varphi}\right)\right)\right] \\
& +\frac{g_{2}^{4}}{\left(16 \pi^{2}\right)^{2} \epsilon^{2}} \mathcal{J}\left(j_{\varphi}\right)\left[\frac{\xi_{W}^{2}}{2}+\frac{3 \xi_{W}}{2}-\frac{43}{4}+2 n_{g}\right. \\
& \left.+\mathcal{J}\left(j_{\varphi}\right)\left(\frac{\mathcal{D}\left(j_{\varphi}\right)}{6}+\frac{\xi_{W}^{2}}{2}-3 \xi_{W}+\frac{9}{2}\right)\right] \\
& -\frac{3}{8} \frac{g_{1}^{2} g_{2}^{2}}{\left(16 \pi^{2}\right)^{2} \epsilon} Y_{\varphi}^{2} \mathcal{J}\left(j_{\varphi}\right)+\frac{g_{1}^{2} g_{2}^{2}}{\left(16 \pi^{2}\right)^{2} \epsilon^{2}}\left[\frac{Y_{\varphi}^{2}}{4} \mathcal{J}\left(j_{\varphi}\right)\left(9-3\left(\xi_{W}+\xi_{B}\right)+\xi_{W} \xi_{B}\right)\right] \\
& -\frac{1}{8} \sum_{J}^{\prime} \frac{\left(\lambda_{\varphi}^{(J)}\right)^{2}}{\left(16 \pi^{2}\right)^{2} \epsilon} \frac{\mathcal{D}(J)}{\mathcal{D}\left(j_{\varphi}\right)}-\frac{1}{32} \frac{\lambda_{\varphi H}^{2}}{\left(16 \pi^{2}\right)^{2} \epsilon}-\frac{\mathcal{J}\left(j_{\varphi}\right)}{128} \frac{\left(\lambda_{\varphi H}^{\prime}\right)^{2}}{\left(16 \pi^{2}\right)^{2} \epsilon},
\end{aligned}
$$




$$
\begin{aligned}
& \delta Z_{H}^{(2)}= \frac{g_{1}^{4}}{\left(16 \pi^{2}\right)^{2} \epsilon}\left[-\frac{1}{192}\left(31+11 \mathcal{D}\left(j_{\varphi}\right) Y_{\varphi}^{2}\right)-\frac{25 n_{g}}{36}\right] \\
&+\frac{g_{1}^{4}}{\left(16 \pi^{2}\right)^{2} \epsilon^{2}}\left[\frac{1}{32}\left(11-6 \xi_{B}+\xi_{B}^{2}+\mathcal{D}\left(j_{\varphi}\right) Y_{\varphi}^{2}\right)+\frac{5 n_{g}}{6}\right] \\
&+\frac{g_{2}^{4}}{\left(16 \pi^{2}\right)^{2} \epsilon}\left[\frac{511}{64}-\frac{3}{2} \xi_{W}-\frac{3}{16} \xi_{W}^{2}-\frac{5 n_{g}}{4}-\frac{11}{48} \mathcal{J}\left(j_{\varphi}\right) \mathcal{D}\left(j_{\varphi}\right)\right] \\
&+\frac{g_{2}^{4}}{\left(16 \pi^{2}\right)^{2} \epsilon^{2}}\left[-\frac{177}{32}+\frac{21}{32} \xi_{W}^{2}-\frac{9}{16} \xi_{W}+\frac{3 n_{g}}{2}+\frac{\mathcal{J}\left(j_{\varphi}\right) \mathcal{D}\left(j_{\varphi}\right)}{8}\right] \\
&-\frac{9}{32} \frac{g_{1}^{2} g_{2}^{2}}{\left(16 \pi^{2}\right)^{2} \epsilon}+\frac{1}{16} \frac{g_{1}^{2} g_{2}^{2}}{\left(16 \pi^{2}\right)^{2} \epsilon^{2}}\left(27-9\left(\xi_{W}+\xi_{B}\right)+3 \xi_{W} \xi_{B}\right) \\
&-\frac{3}{16} \frac{\lambda_{H}^{2}}{\left(16 \pi^{2}\right)^{2} \epsilon}-\frac{\mathcal{D}\left(j_{\varphi}\right)}{64} \frac{\lambda_{\varphi H}^{2}}{\left(16 \pi^{2}\right)^{2} \epsilon}-\frac{\mathcal{D}\left(j_{\varphi}\right) \mathcal{J}\left(j_{\varphi}\right)}{256} \frac{\left(\lambda_{\varphi H}^{\prime}\right)^{2}}{\left(16 \pi^{2}\right)^{2} \epsilon} \\
&+\frac{27 y_{t}^{4}+27 y_{b}^{4}+27 y_{c}^{4}-6 y_{t}^{2} y_{b}^{2}+9 y_{\tau}^{4}}{8\left(16 \pi^{2}\right)^{2} \epsilon}-\frac{9 y_{t}^{4}+9 y_{b}^{4}+9 y_{c}^{4}-18 y_{t}^{2} y_{b}^{2}+3 y_{\tau}^{4}}{4\left(16 \pi^{2}\right)^{2} \epsilon^{2}} \\
&-\frac{10 g_{s}^{2}}{\left(16 \pi^{2}\right)^{2} \epsilon}\left(y_{t}^{2}+y_{b}^{2}+y_{c}^{2}\right)+\frac{12 g_{s}^{2}}{\left(16 \pi^{2}\right)^{2} \epsilon^{2}}\left(y_{t}^{2}+y_{b}^{2}+y_{c}^{2}\right) \\
&-\frac{15}{16} \frac{g_{2}^{2}}{\left(16 \pi^{2}\right)^{2} \epsilon}\left(3 y_{t}^{2}+3 y_{b}^{2}+3 y_{c}^{2}+y_{\tau}^{2}\right) \\
&-\frac{g_{2}^{2}}{8\left(16 \pi^{2}\right)^{2} \epsilon^{2}}\left(3 y_{t}^{2}+3 y_{b}^{2}+3 y_{c}^{2}+y_{\tau}^{2}\right)\left(9-6 \xi_{W}\right) \\
&-\frac{g_{1}^{2}}{48\left(16 \pi^{2}\right)^{2} \epsilon}\left(85 y_{t}^{2}+25 y_{b}^{2}+85 y_{c}^{2}+75 y_{\tau}^{2}\right) \\
&+\frac{g_{1}^{2}}{8\left(16 \pi^{2}\right)^{2} \epsilon^{2}}\left[\left(6 y_{t}^{2}+6 y_{b}^{2}+6 y_{c}^{2}+2 y_{\tau}^{2}\right) \xi_{B}-\left(y_{t}^{2}+13 y_{b}^{2}+y_{c}^{2}-9 y_{\tau}^{2}\right)\right] \\
&
\end{aligned}
$$

For the two-loop contributions to the electroweak gauge-boson field renormalization constants we consider only contributions of the scalar multiplet. We find

$$
\begin{aligned}
\delta Z_{B}^{(2)}= & -\frac{1}{8} \frac{g_{1}^{4}}{\left(16 \pi^{2}\right)^{2} \epsilon} Y_{\varphi}^{4} \mathcal{D}\left(j_{\varphi}\right)-\frac{1}{2} \frac{g_{1}^{2} g_{2}^{2}}{\left(16 \pi^{2}\right)^{2} \epsilon} Y_{\varphi}^{2} \mathcal{J}\left(j_{\varphi}\right) \mathcal{D}\left(j_{\varphi}\right) \\
\delta Z_{W}^{(2)}= & \frac{g_{2}^{4}}{\left(16 \pi^{2}\right)^{2} \epsilon}\left(\frac{1}{12} \mathcal{J}\left(j_{\varphi}\right) \mathcal{D}\left(j_{\varphi}\right)-\frac{2}{3} \mathcal{J}\left(j_{\varphi}\right)^{2} \mathcal{D}\left(j_{\varphi}\right)\right) \\
& +\frac{g_{2}^{4}}{\left(16 \pi^{2}\right)^{2} \epsilon^{2}} \frac{1}{18} \mathcal{J}\left(j_{\varphi}\right) \mathcal{D}\left(j_{\varphi}\right)\left(3+2 \xi_{W}\right)-\frac{g_{1}^{2} g_{2}^{2}}{\left(16 \pi^{2}\right)^{2} \epsilon} \frac{1}{6} Y_{\varphi}^{2} \mathcal{J}\left(j_{\varphi}\right) \mathcal{D}\left(j_{\varphi}\right)
\end{aligned}
$$


Open Access. This article is distributed under the terms of the Creative Commons Attribution License (CC-BY 4.0), which permits any use, distribution and reproduction in any medium, provided the original author(s) and source are credited.

\section{References}

[1] M. Cirelli, N. Fornengo and A. Strumia, Minimal dark matter, Nucl. Phys. B 753 (2006) 178 [hep-ph/0512090] [INSPIRE].

[2] J.C. Collins, Renormalization: An Introduction to Renormalization, The Renormalization Group, and the Operator Product Expansion, Cambridge Monographs on Mathematical Physics, vol. 26, Cambridge University Press, Cambridge (1986) [DOI] [InSPIRE].

[3] F. Bishara, J. Brod, B. Grinstein and J. Zupan, Renormalization Group Effects in Dark Matter Interactions, JHEP 03 (2020) 089 [arXiv:1809.03506] [INSPIRE].

[4] J. Brod, A. Gootjes-Dreesbach, M. Tammaro and J. Zupan, Effective Field Theory for Dark Matter Direct Detection up to Dimension Seven, JHEP 10 (2018) 065 [arXiv:1710.10218] [INSPIRE].

[5] V. Silveira and A. Zee, Scalar phantoms, Phys. Lett. B 161 (1985) 136 [InSPIRE].

[6] J. McDonald, Gauge singlet scalars as cold dark matter, Phys. Rev. D 50 (1994) 3637 [hep-ph/0702143] [INSPIRE].

[7] C.P. Burgess, M. Pospelov and T. ter Veldhuis, The Minimal model of nonbaryonic dark matter: A Singlet scalar, Nucl. Phys. B 619 (2001) 709 [hep-ph/0011335] [INSPIRE].

[8] W. Chao, G.-J. Ding, X.-G. He and M. Ramsey-Musolf, Scalar Electroweak Multiplet Dark Matter, JHEP 08 (2019) 058 [arXiv: 1812.07829] [INSPIRE].

[9] M.E. Machacek and M.T. Vaughn, Two Loop Renormalization Group Equations in a General Quantum Field Theory. 3. Scalar Quartic Couplings, Nucl. Phys. B 249 (1985) 70 [inSPIRE].

[10] I. Jack and H. Osborn, General Background Field Calculations With Fermion Fields, Nucl. Phys. B 249 (1985) 472 [inSPIRE].

[11] H. Arason et al., Renormalization group study of the standard model and its extensions. 1. The Standard model, Phys. Rev. D 46 (1992) 3945 [InSPIRE].

[12] M.-x. Luo, H.-w. Wang and Y. Xiao, Two loop renormalization group equations in general gauge field theories, Phys. Rev. D 67 (2003) 065019 [hep-ph/0211440] [INSPIRE].

[13] M.-x. Luo and Y. Xiao, Two loop renormalization group equations in the standard model, Phys. Rev. Lett. 90 (2003) 011601 [hep-ph/0207271] [INSPIRE].

[14] K.G. Chetyrkin and M.F. Zoller, Three-loop $\beta$-functions for top-Yukawa and the Higgs self-interaction in the Standard Model, JHEP 06 (2012) 033 [arXiv:1205.2892] [INSPIRE].

[15] L.N. Mihaila, J. Salomon and M. Steinhauser, Renormalization constants and $\beta$-functions for the gauge couplings of the Standard Model to three-loop order, Phys. Rev. D 86 (2012) 096008 [arXiv: 1208.3357] [INSPIRE].

[16] A.V. Bednyakov, A.F. Pikelner and V.N. Velizhanin, Three-loop Higgs self-coupling $\beta$-function in the Standard Model with complex Yukawa matrices, Nucl. Phys. B 879 (2014) 256 [arXiv:1310.3806] [INSPIRE]. 
[17] A.V. Bednyakov, A.F. Pikelner and V.N. Velizhanin, Three-loop SM $\beta$-functions for matrix Yukawa couplings, Phys. Lett. B 737 (2014) 129 [arXiv:1406.7171] [INSPIRE].

[18] J. Davies, F. Herren, C. Poole, M. Steinhauser and A.E. Thomsen, Gauge Coupling $\beta$ Functions to Four-Loop Order in the Standard Model, Phys. Rev. Lett. 124 (2020) 071803 [arXiv: 1912.07624] [INSPIRE].

[19] S. Weinberg, Lectures on Quantum Mechanics, 2 edition, Cambridge University Press (2015) [DOI].

[20] K.G. Chetyrkin, M. Misiak and M. Münz, $\beta$-functions and anomalous dimensions up to three loops, Nucl. Phys. B 518 (1998) 473 [hep-ph/9711266] [INSPIRE].

[21] J.A.M. Vermaseren, New features of FORM, math-ph/0010025 [INSPIRE].

[22] A.I. Davydychev and J.B. Tausk, Two loop selfenergy diagrams with different masses and the momentum expansion, Nucl. Phys. B 397 (1993) 123 [INSPIRE].

[23] P. Nogueira, Automatic Feynman graph generation, J. Comput. Phys. 105 (1993) 279.

[24] A. Messiah, 9j symbols, in Quantum Mechanics Vol. II, ch. Appendix C III, pp. 1066-1068, North-Holland, Amsterdam, Netherlands (1962).

[25] T.L. Trueman, Spurious anomalies in dimensional renormalization, Z. Phys. C 69 (1996) 525 [hep-ph/9504315] [INSPIRE].

[26] L. Sartore and I. Schienbein, PyR@TE 3, arXiv:2007.12700 [InSPIRE].

[27] Particle Data Group collaboration, Review of Particle Physics, Prog. Theor. Exp. Phys 2020 (2020) 083C01.

[28] G. Degrassi et al., Higgs mass and vacuum stability in the Standard Model at NNLO, JHEP 08 (2012) 098 [arXiv: 1205.6497] [INSPIRE].

[29] H.T. Johansson and C. Forssén, Fast and accurate evaluation of Wigner 3j, 6j, and 9j symbols using prime factorisation and multi-word integer arithmetic, SIAM J. Sci. Statist. Comput. 38 (2016) A376 [arXiv:1504.08329] [INSPIRE].

[30] A. Wodtke and J. Halpern, Mathematica notebook to calculate Wigner 9-j Symbols, December (1999) [https://library.wolfram.com/infocenter/MathSource/481/].

[31] M. Cirelli and A. Strumia, Minimal Dark Matter: Model and results, New J. Phys. 11 (2009) 105005 [arXiv: 0903.3381] [INSPIRE].

[32] T. Muta, Foundations of Quantum Chromodynamics: An Introduction to Perturbative Methods in Gauge Theories (3rd ed.), World scientific Lecture Notes in Physics, vol. 78, World Scientific, Hackensack, NJ (2010) [INSPIRE]. 